


\title{
MESH-FREE MODELING OF SPATIALLY \\ INHOMOGENEOUS AEROSOLS IN ARBITRARY \\ GEOMETRIES
}

A dissertation presented to the

Faculty of the Graduate School, University of Missouri

\author{
In Partial Fulfillment \\ Of the Requirements for the Degree
}

Doctor of Philosophy in Nuclear Engineering

\section{By \\ MATTHEW BORAAS}

Dr. Sudarshan Loyalka, Dissertation Supervisor

May 2018 
The undersigned, appointed by the Dean of the Graduate School, have examined the dissertation entitled

\title{
MESH-FREE MODELING OF SPATIALLY INHOMOGENEOUS AEROSOLS IN ARBITRARY GEOMETRIES
}

\author{
Presented by Matthew Boraas \\ A candidate for the degree of Doctor of Philosophy \\ in Nuclear Engineering
}

And hereby certify that, in their opinion, it is worthy of acceptance.

Dr. Sudarshan Loyalka

Dr. Mark Prelas

Dr. Robert Tompson

Dr. Tushar Ghosh

Dr. Stephen Montgomery-Smith 


\section{Dedicated to my \\ beautiful wife Daniela. \\ Thank you for your \\ love and support!}

Ialso want to lift up thanks to my

Cord and Pavior, Fesus Ghrist.

Qf fou have faithfully led me through it all.

Jo Ofou be the glory! 


\section{ACKNOWLEDGMENTS}

I would like to thank my advisor, Dr. Sudarshan Loyalka, for the tremendous amount of time and effort you have invested in me. Your willingness to encourage and advise me in my work has been critical to my success. The generosity you have exhibited during my time at the University of Missouri has been overwhelming, and I am grateful to have an advisor that cares so strongly for the wellbeing of his students.

Another "thank you" must be given to my doctoral committee. This committee consists of doctors Sudarshan Loyalka, Mark Prelas, Robert Tompson, Tushar Ghosh, and Stephen Montgomery-Smith. Your vote of confidence in me is most encouraging.

I would also like to thank Latricia Vaughn and James Bennett. While you operate behind the scenes, it has been proven time and again that the Nuclear Science and Engineering Institute cannot operate without you. The immense amount of work you did on my behalf is extremely appreciated.

Last, a special thanks must be extended to my undergraduate professor Patrick Tebbe at Minnesota State University, Mankato. You were responsible for introducing me to the field of nuclear engineering and to the Nuclear Science and Engineering Institute at the University of Missouri. Without your keen recommendation I would never have thought of pursuing my degree. 


\section{TABLE OF CONTENTS}

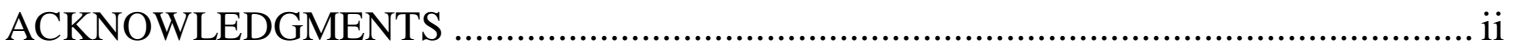

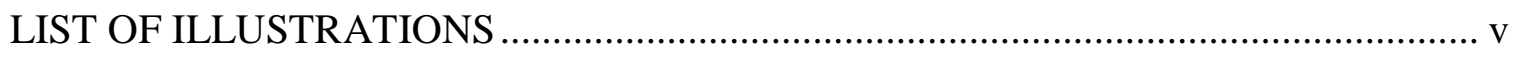

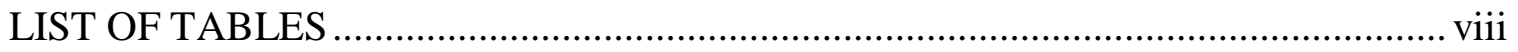

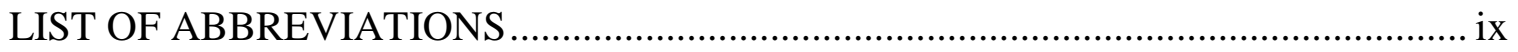

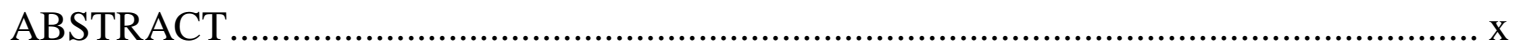

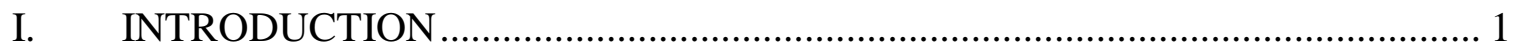

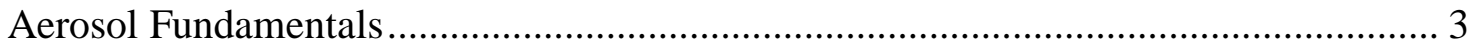

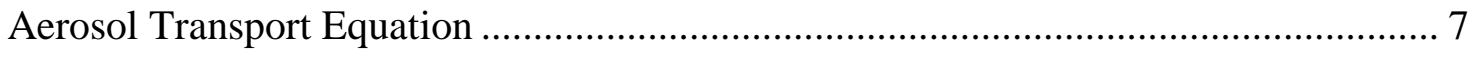

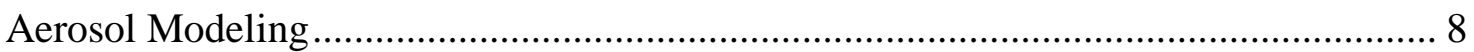

Scope and Organization of Research................................................................... 11

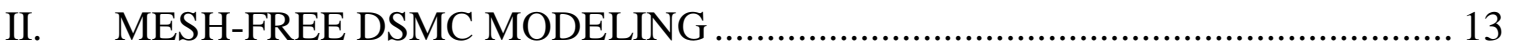

III. DIFFUSION, DEPOSITION, COAGULATION, AND CONDENSATION

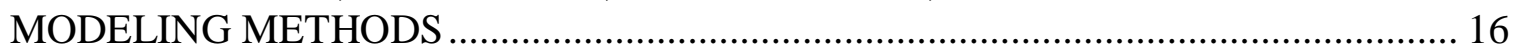

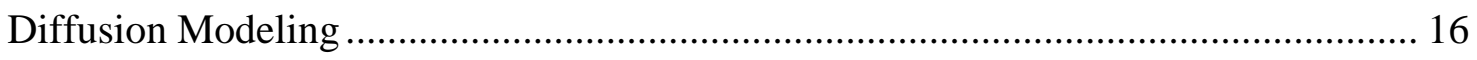

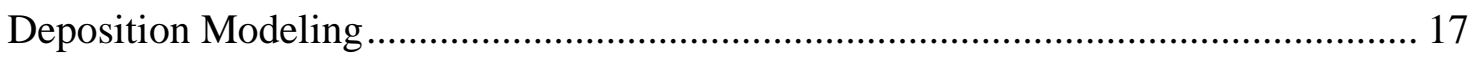

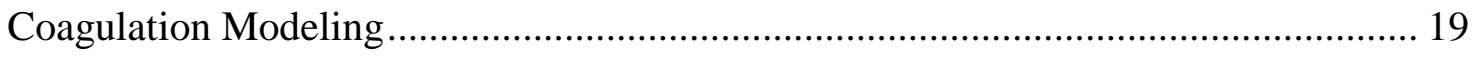

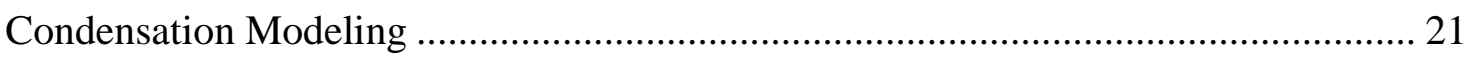

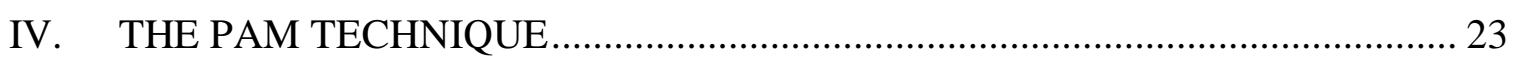

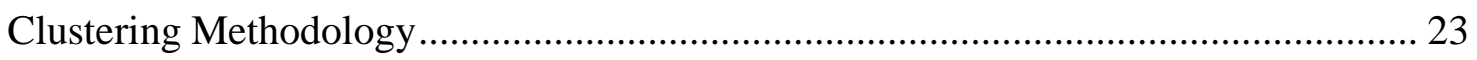

The PAM Algorithm ............................................................................................. 28

V. PROGRAM DEVELOPMENT AND MODIFICATIONS ................................ 33

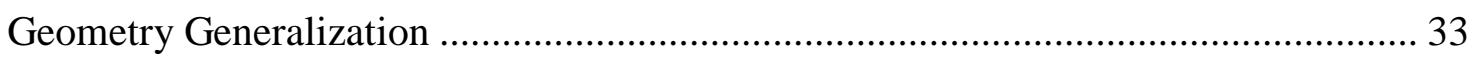

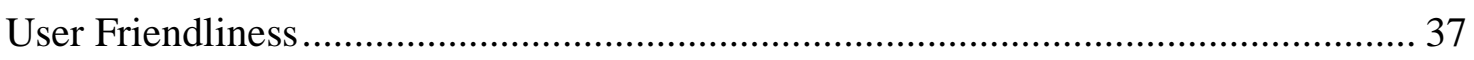

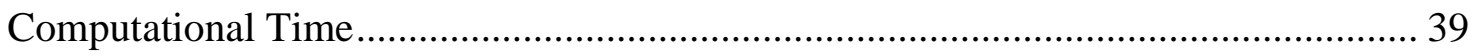

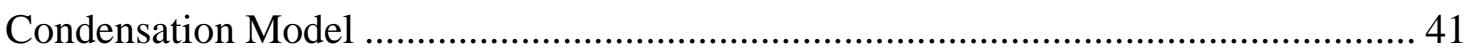

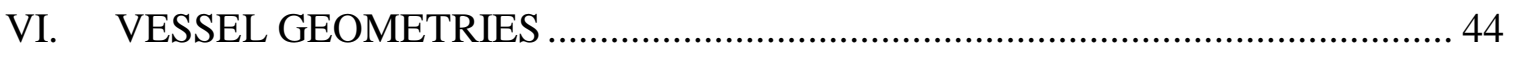

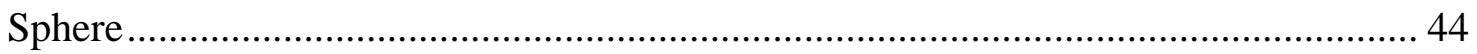

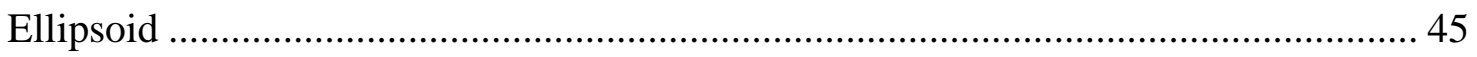

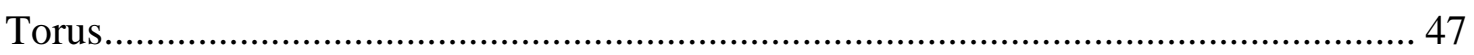

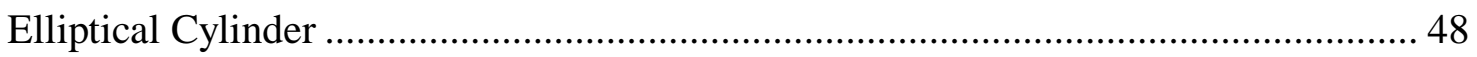

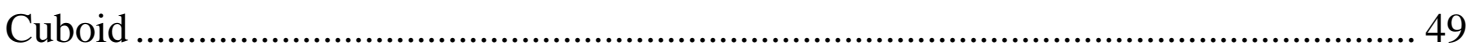

Sphere with Internal Obstruction ................................................................... 51 


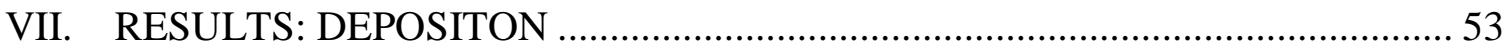

Sphere

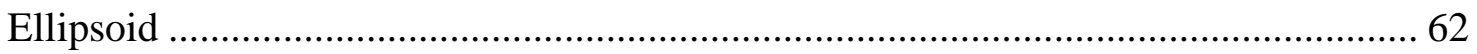

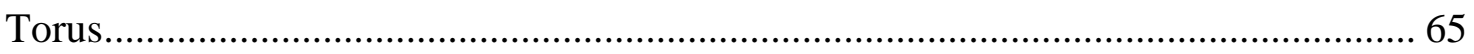

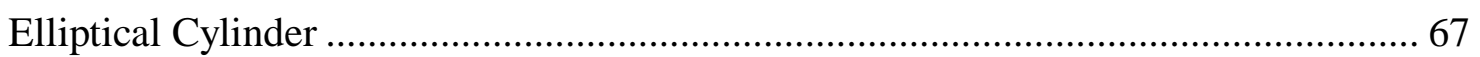

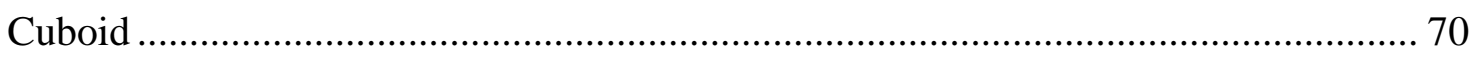

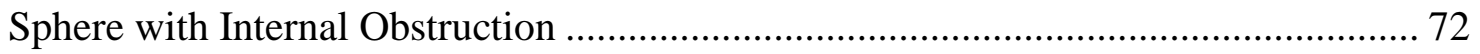

VIII. RESULTS: DEPOSITON AND COAGULATION …………………………...... 75

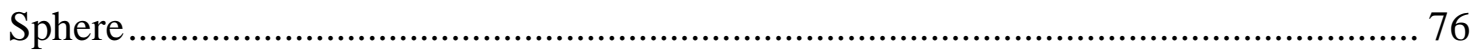

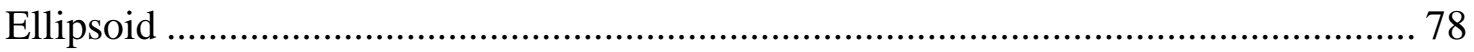

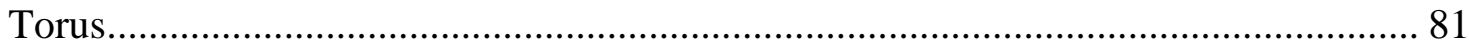

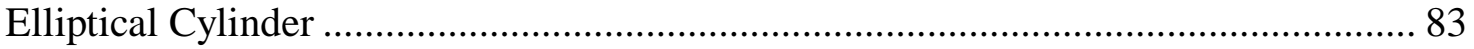

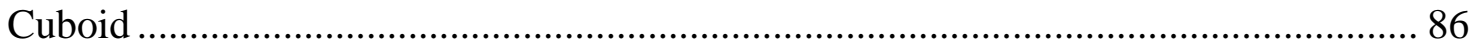

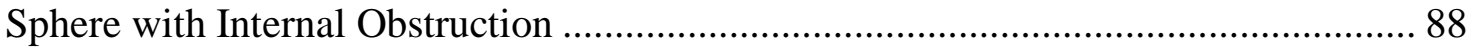

IX. RESULTS: DEPOSITON, COAGULATION, AND CONDENSATION ……..... 91

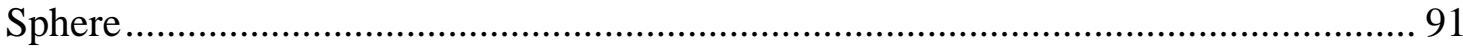

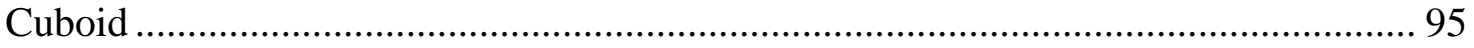

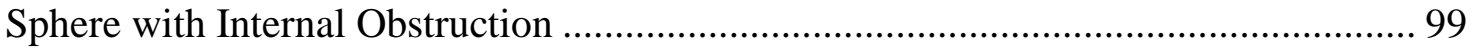

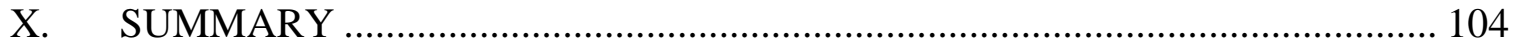

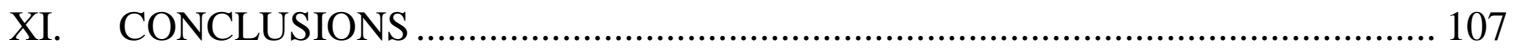

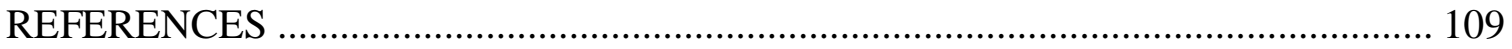

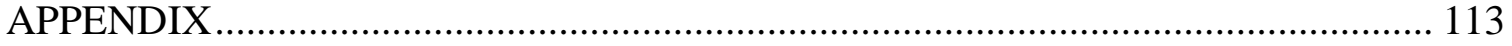

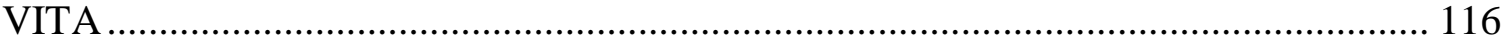




\section{LIST OF ILLUSTRATIONS}

Figure:

Page:

Figure 1: Typical Particle Sizes, in Micrometers $(\mu \mathrm{m})$............................................ 4

Figure 2: Aerosol Dynamics Processes............................................................... 5

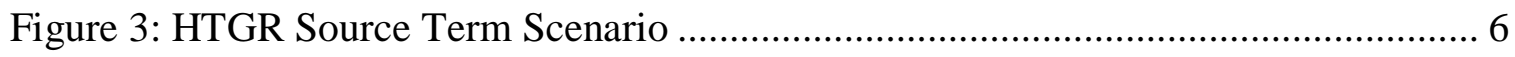

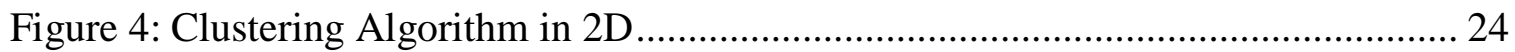

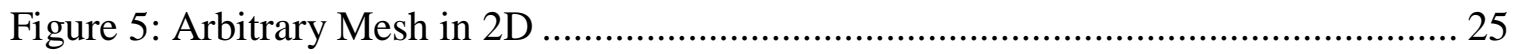

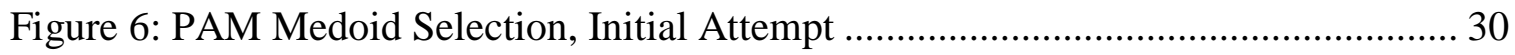

Figure 7: PAM Medoid Selection, Revised Attempt ................................................. 31

Figure 8: Spherical Vessel with Aerosol Particles.................................................... 45

Figure 9: Elliptical Vessel with Aerosol Particles ...................................................... 46

Figure 10: Toroid Vessel with Aerosol Particles ........................................................ 47

Figure 11: Cylindrical Vessel with Aerosol Particles .................................................. 49

Figure 12: Cuboid Vessel with Aerosol Particles ...................................................... 50

Figure 13: Spherical Vessel with Internal Obstruction and Aerosol Particles................. 52

Figure 14: Comparison of Initial Aerosol Particle Size Distribution ............................. 54

Figure 15: A Sphere of Radius R, Split Into Ten Shells ........................................... 56

Figure 16: Total Particle Mass Suspended in Each Shell at 50 Seconds ........................ 57

Figure 17: Total Particle Mass Suspended in Each Shell at 100 Seconds ........................ 58

Figure 18: Mass Concentration at 50 Seconds, Using Spherical Symmetry ................... 59

Figure 19: Mass Concentration at 50 Seconds, Using Sample Spheres .......................... 59

Figure 20: Mass Concentration at 100 Seconds, Using Spherical Symmetry .................. 60

Figure 21: Mass Concentration at 100 Seconds, Using Sample Spheres .......................... 60

Figure 22: Cumulative Particle Deposition on Inner Surface Of Sphere, for DSMC (Line) and FEM (Points) 


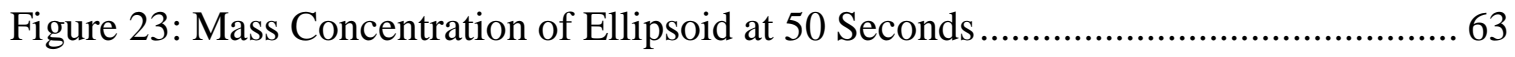

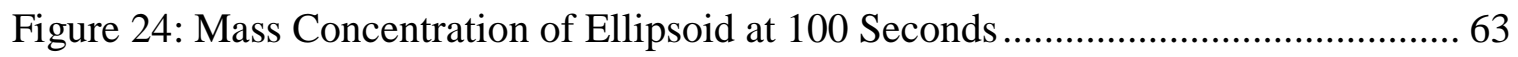

Figure 25: Cumulative Particle Deposition on Inner Surface of Ellipsoid, for DSMC

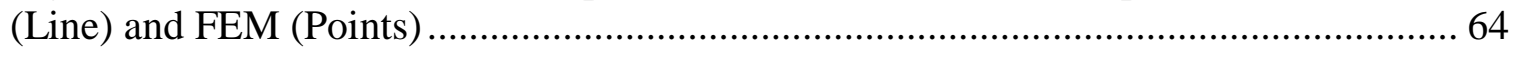

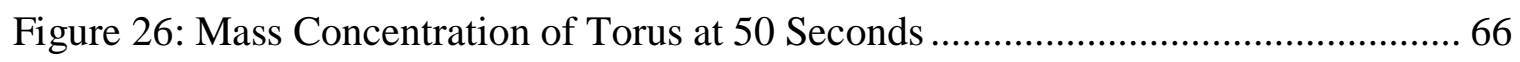

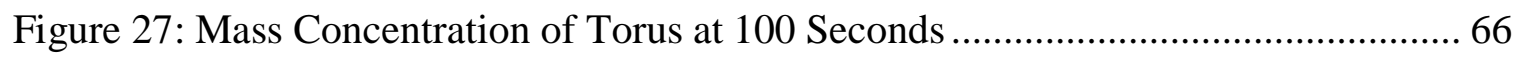

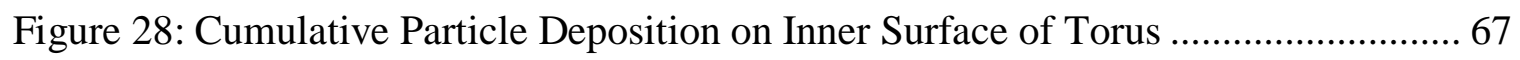

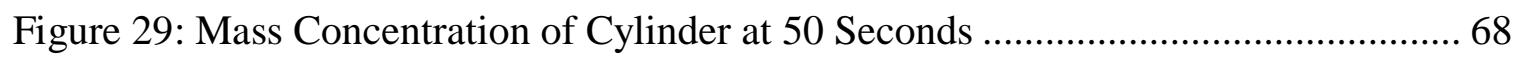

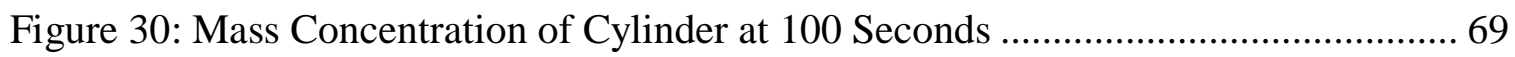

Figure 31: Cumulative Particle Deposition on Inner Surface of Cylinder....................... 69

Figure 32: Mass Concentration of Cuboid at 50 Seconds........................................... 70

Figure 33: Mass Concentration of Cuboid at 100 Seconds......................................... 71

Figure 34: Cumulative Particle Deposition on Inner Surface of Cuboid.......................... 71

Figure 35: Mass Concentration of Sphere with Internal Obstruction at 50 Seconds ........ 73

Figure 36: Mass Concentration of Sphere with Internal Obstruction at 100 Seconds...... 73

Figure 37: Cumulative Particle Deposition on Inner Surfaces of Sphere with Internal

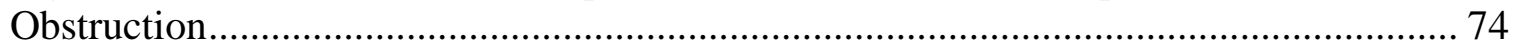

Figure 38: Suspended Mass in Sphere at 10 Seconds ................................................ 77

Figure 39: Suspended Mass in Sphere at 20 Seconds ............................................... 77

Figure 40: Cumulative Particle Deposition on Inner Surface of Sphere ........................ 78

Figure 41: Suspended Mass in Ellipsoid at 10 Seconds .......................................... 79

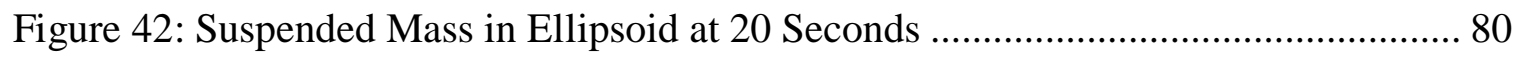

Figure 43: Cumulative Particle Deposition on Inner Surface of Ellipsoid ...................... 80

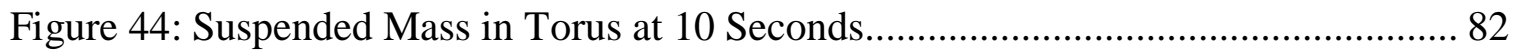

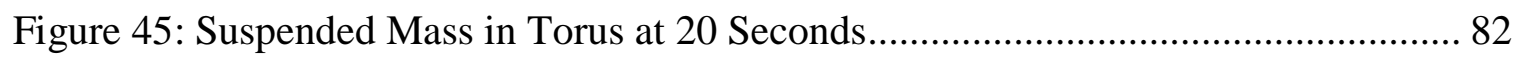

Figure 46: Cumulative Particle Deposition on Inner Surface of Torus ........................ 83 
Figure 47: Suspended Mass in Cylinder at 10 Seconds ............................................ 84

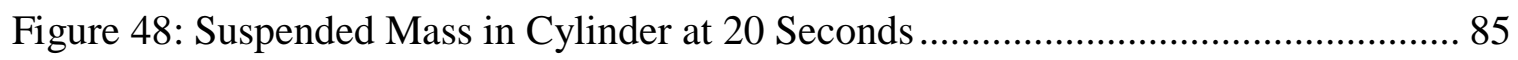

Figure 49: Cumulative Particle Deposition on Inner Surface of Cylinder....................... 85

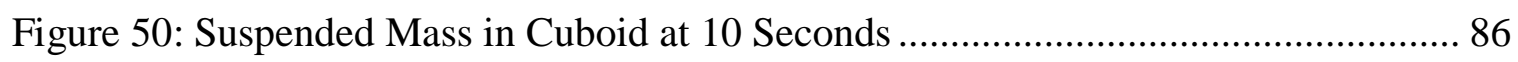

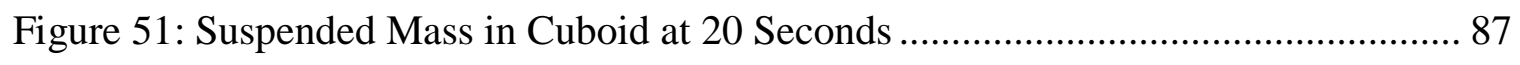

Figure 52: Cumulative Particle Deposition on Inner Surface of Cuboid......................... 87

Figure 53: Suspended Mass in Sphere with Internal Obstruction at 10 Seconds ............ 89

Figure 54: Suspended Mass in Sphere with Internal Obstruction at 20 Seconds ............. 89

Figure 55: Cumulative Particle Deposition on Inner Surfaces of Sphere with Internal

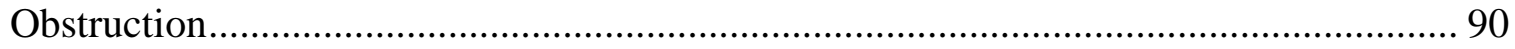

Figure 56: Mass Concentration of Sphere at 10 Seconds ....................................... 92

Figure 57: Mass Concentration of Sphere at 20 Seconds .......................................... 93

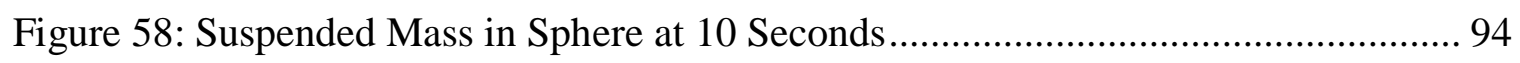

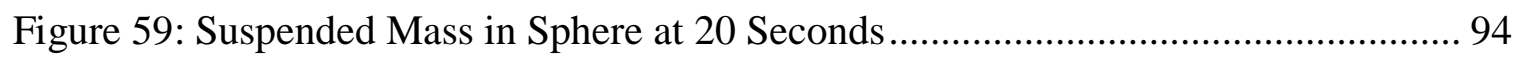

Figure 60: Total Condensation Inside of the Sphere ............................................... 95

Figure 61: Mass Concentration of Cuboid at 10 Seconds.......................................... 96

Figure 62: Mass Concentration of Cuboid at 20 Seconds............................................ 97

Figure 63: Suspended Mass in Cuboid at 10 Seconds ............................................. 98

Figure 64: Suspended Mass in Cuboid at 20 Seconds ............................................... 98

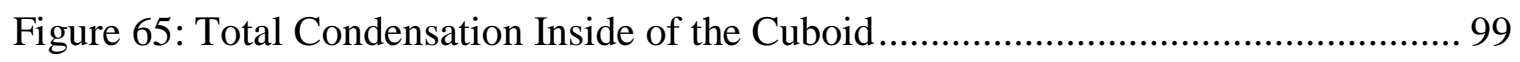

Figure 66: Mass Concentration of Sphere with Internal Obstruction at 10 Seconds ...... 100

Figure 67: Mass Concentration of Sphere with Internal Obstruction at 20 Seconds ...... 101

Figure 68: Suspended Mass in Sphere with Internal Obstruction at 10 Seconds ........... 102

Figure 69: Suspended Mass in Sphere with Internal Obstruction at 20 Seconds ........... 102

Figure 70: Total Condensation Inside of the Sphere with Internal Obstruction ............. 103 


\section{LIST OF TABLES}

Table:

Page:

Table 1: Total Distance Calculations for Initial and Revised Attempts ........................ 32

Table 2: Initial Parameters for All DSMC Simulations................................................ 54

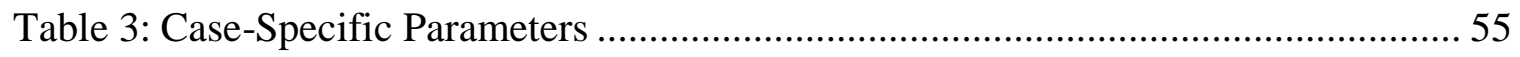

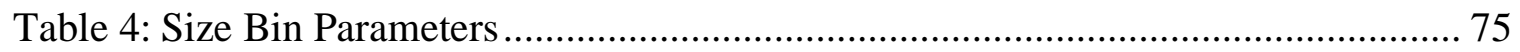

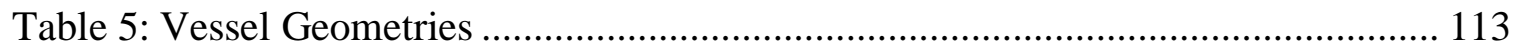




\title{
LIST OF ABBREVIATIONS
}

\author{
Abbreviation: Definition: \\ DSMC ….................... Direct Simulation Monte Carlo \\ FEM .........................Finite Element Method \\ GUI ........................Graphical User Interface \\ HTGR .......................High Temperature Gas-Cooled Reactor \\ LACE ........................Light-Water Reactor Aerosol Containment Experiment \\ NTC.............................No Time Counter \\ PAM .........................Partitioning Around Medoids
}




\title{
MESH-FREE MODELING OF SPATIALLY INHOMOGENEOUS AEROSOLS IN ARBITRARY GEOMETRIES
}

\author{
Matthew Boraas \\ Dr. Sudarshan Loyalka, Advisor \\ ABSTRACT
}

Many issues affect the nuclear source term, one of which is the existence of aerosols. These aerosols are usually simulated with the assumption that they are spatially homogeneous. Making this assumption reduces complexity when compared to spatially inhomogeneous aerosol models.

This dissertation investigates using the Direct Simulation Monte Carlo (DSMC) method for spatially inhomogeneous aerosol modeling. To do so, a mesh-free DSMC alternative was used that takes advantage of Euclidian distance in its clustering algorithm. Deposition, coagulation, and condensation cases were addressed for aerosols inside several fundamental and complex geometries. The first model for each geometry was deposition only, and was run for 100 simulated seconds. Results were compared to previous literature and other techniques, and were found to match closely. Coagulation was then incorporated into the model, and the program was run for 20 simulated seconds. As the model progressed in time, the average mass of the suspended aerosol particles grew. This indicated the presence of coagulation. Lastly, an isothermal Mason condensation model was added to the code. Again, the program was run for 20 simulated seconds. Particle mass increased dramatically, demonstrating condensation effects. 


\section{INTRODUCTION}

While many issues affect the composition and quantity of the nuclear source term, one significant factor is the existence of aerosols in the containment structure or in the primary reactor vessel itself $^{1-12}$. This significance has led to a detailed investigation of aerosol behavior, such as analysis of particle charge and ion distribution ${ }^{13}$ as well as studies on the effects of coagulation and growth through heterogeneous condensation ${ }^{14}$. Experimental work on aerosol behavior has also been done. For example, in the series of Light-Water Reactor Aerosol Containment Experiments (LACE) studies, a detailed investigation into aerosol retention in the reactor coolant system piping and primary containment was conducted ${ }^{15-19}$. Unfortunately, while analyses such as these have greatly improved the understanding of aerosols ${ }^{20-32}$, this understanding is by no means complete: Many obstacles and limiting assumptions remain in place $^{8}$. One such restriction is in regards to aerosol distribution, which is commonly considered to be spatially homogeneous. This assumption greatly reduces computational complexity, which is why most studies have not involved an analysis of the effects of spatial inhomogeneity on $\operatorname{aerosols}^{33,34}$.

The purpose of this dissertation is to expand understanding of the behavior of spatially inhomogeneous aerosols using the Direct Simulation Monte Carlo (DSMC) method $^{35-37}$. Specifically, this dissertation presents the results of a study analyzing aerosol deposition, coagulation, and condensation in a series of spatially inhomogeneous environments. These environments primarily consist of fundamental geometries $-\mathrm{a}$ sphere, ellipsoid, torus, elliptical cylinder, and cuboid. However, the modeling was also extended to the more complex case of a spherical geometry containing an internal 
obstruction. The ultimate goal is to pursue DSMC-based aerosol modeling inside reactor shapes.

In order to facilitate computations, it was necessary to utilize a nontraditional application of the DSMC method. Conventionally, DSMC is applied to a computational domain that has been divided into a mesh (a number of fixed cells). The positions of all the particles within the domain change with every time step, and then those that reside within any given cell are analyzed for particle interactions. This evaluation is done with the No Time Counter (NTC) technique ${ }^{37}$. However, the construction of the mesh can quickly become computationally demanding as well as extremely labor intensive, depending on the enclosing geometric boundaries of the computational domain. Due to the spatially inhomogeneous nature of the aerosol environment, it was determined that such an approach would be too computationally expensive. Fortunately, in Bird's original 1976 text $^{36}$, he does suggest a categorization method wherein cells are given a representative geometric point. Particles can be considered to reside inside that cell when its distance to a point is less than the distance to any other representative geometric point.

To efficiently model spatially inhomogeneous aerosols, it was decided to employ a similar technique to the one suggested by Bird. This selected process, known as the Partitioning Around Medoids (PAM) method ${ }^{38,39}$, employs a clustering technique and is commonly used for machine learning and group theory purposes. Unlike a more traditional mesh-based modeling approach, the PAM method takes representative points (medoids) and then clusters the particles based on their distance to these points. As the particles progress through time their positions change, necessitating a recalculation of appropriate medoids. This is accomplished in each time step by an optimization 
algorithm that minimizes the total distance between the medoids and the associated particles. It is the intent of this work to further demonstrate the validity of this approach when modeling aerosol interactions.

\section{Aerosol Fundamentals}

Before aerosol modeling can be discussed, it is necessary to understand the fundamental properties and behaviors of the aerosol particles themselves. Most particles have a large surface to volume ratio. A second important property is that they tend to have significant interactions with light. The particles also have the potential for an extremely large aerosol residence time (on the order of months to years), which becomes of particular concern in nuclear reactor accident scenarios. For example, during the Fukushima Daiichi nuclear accident in March 2011, an aerosolized source term released from the facility drifted east across the Pacific. Due to the long aerosol residence time, this became a major concern on the west coast of the United States ${ }^{40-42}$.

Another significant factor about aerosol particles that must be considered is how the particles were generated. This is important because it dictates the physical, chemical, and biological properties of the particles. The method of generation also impacts the sizes of the particles in the aerosol. Typical particle sizes can range from nanometers (nm) to micrometers $(\mu \mathrm{m})$.

In order to properly grasp the size dimensions being discussed, the size ranges of some common particles are provided in Figure 1. Note that lung damage is especially prevalent for aerosol particles $0.1 \mu \mathrm{m}$ to $5 \mu \mathrm{m}$ in diameter. Aerosol particles larger than 5 $\mu \mathrm{m}$ are less dangerous because they tend to deposit earlier in the lungs due to particle momentum. For aerosol particles under $0.1 \mu \mathrm{m}$, lung damage is unlikely because the 
particles have large diffusion coefficients. These large coefficients mean that the particles undergo significant diffusion processes, and consequently deposit earlier in the lungs.

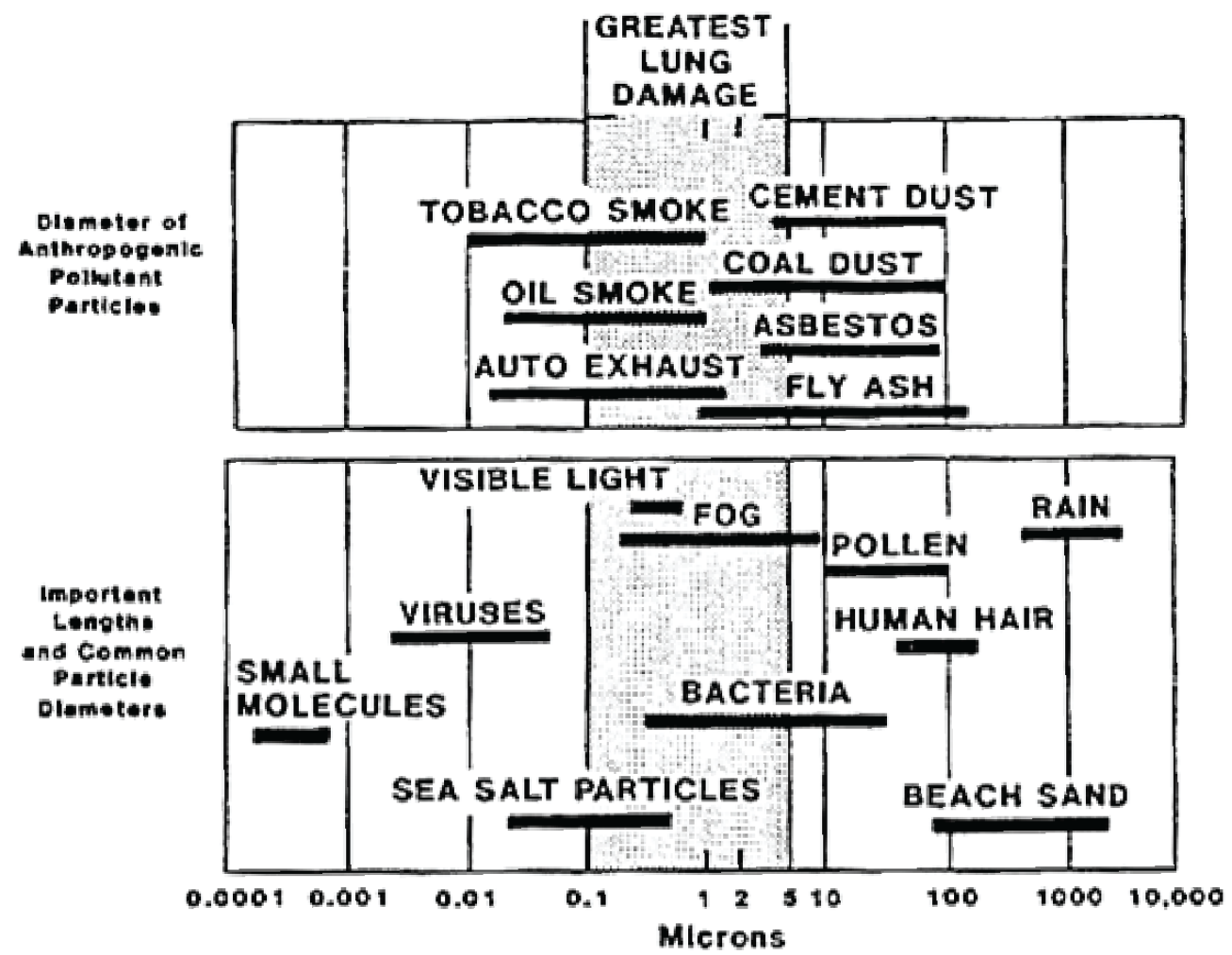

Figure 1: Typical Particle Sizes, in Micrometers $(\mu \mathrm{m})$

In addition to the properties described above, aerosol particles are also influenced by particle interactions and aerosol dynamics. While many different particle interactions can occur, only four will be discussed. The first is an interaction between two molecules, which was not of concern during the research conducted for this dissertation. A second type of interaction is between a molecule and a particle, which can be visualized as similar to the interaction between a ping-pong ball and a bowling ball. It is this interaction that is responsible for Brownian motion in larger particles. Another type of particle interaction is between two particles, and has the potential to result in a coagulation event. The last type of interaction is between a particle and the overall system. Deposition is a wonderful example of the effects of this type of interaction. 
While particle interactions occur on an individual basis, aerosol dynamics looks at phenomena that affects the total aerosol population over time. This includes condensation, Brownian motion, settling, deposition, and resuspension. It also includes particle motion due to turbulence, thermal effects, and diffusive effects. Figure 2 shows these aerosol dynamics phenomena for the case when aerosol particle concentration is much less than the molecular concentration.

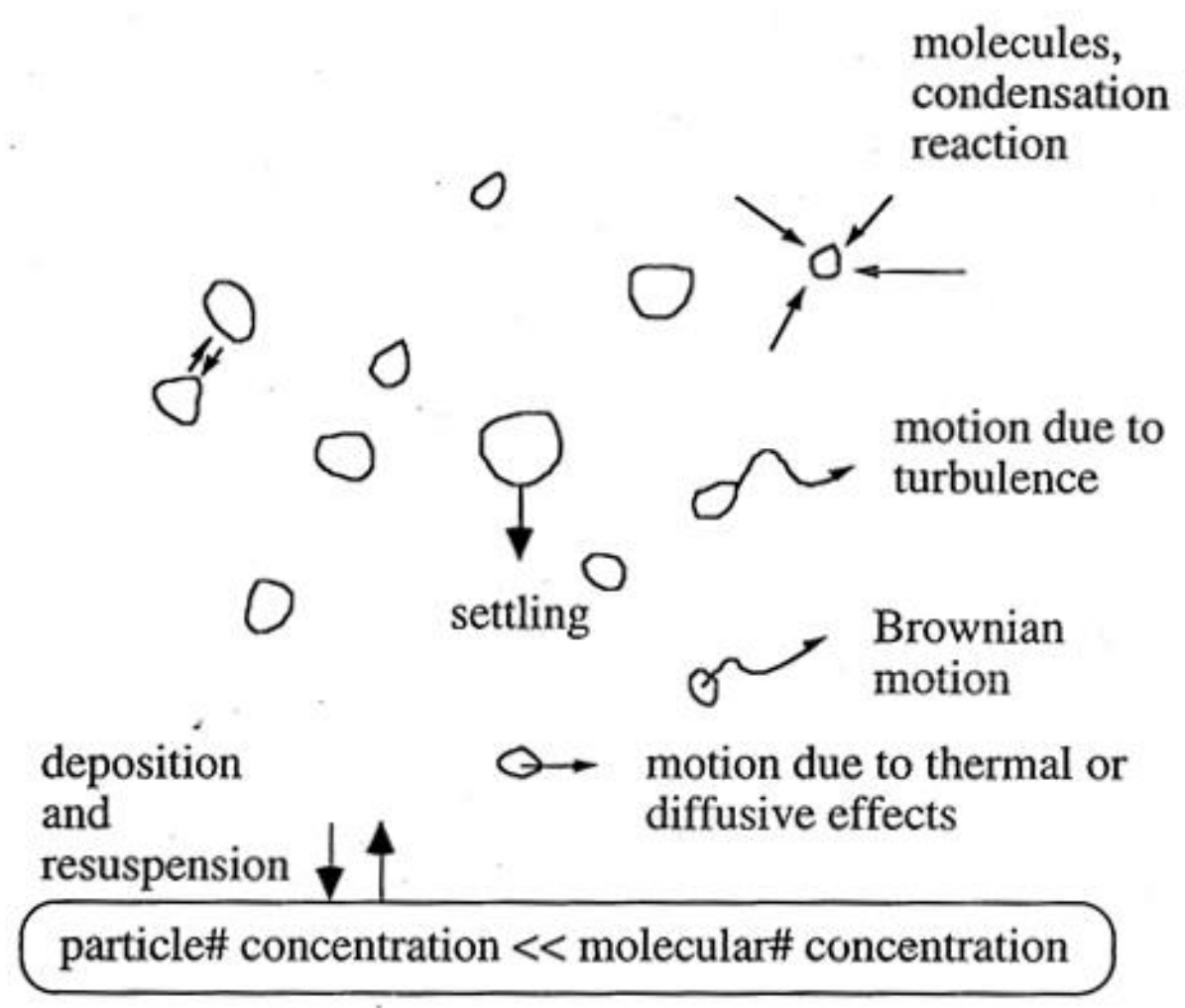

Figure 2: Aerosol Dynamics Processes

Due to the numerous properties of aerosols, they can be extremely advantageous in a wide variety of different applications. For example, aerosols are critically important in the health and medical sciences. These fields traditionally use aerosols as a means of medication delivery to the body. An asthma inhaler is one example of this. Another area in which aerosols are of concern in is pollution and climate change. This is due to the inherent properties of aerosol particles, including their interaction with light. The field of 
meteorological science is also heavily reliant on aerosol particles. Both clouds and rain depend on the presence of these particles in order to form. Another application of aerosol particles is in the synthesis of some materials, where they must be accounted for. Even the scientific discipline of astrophysics has to consider the effects of aerosol particles.

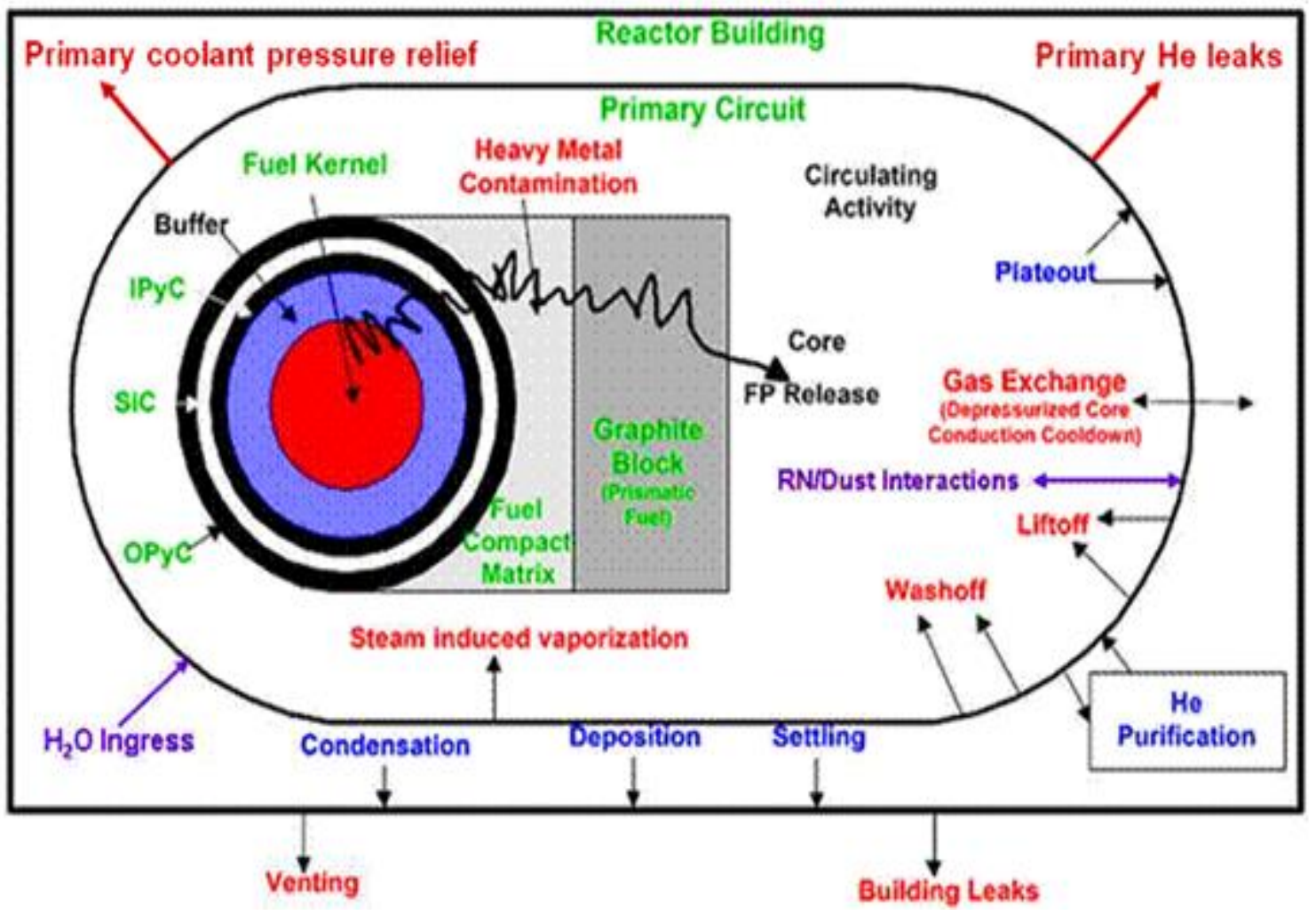

\section{Figure 3: HTGR Source Term Scenario}

In nuclear engineering, aerosol particles must be considered in several different segments of the nuclear fuel cycle. One such segment is during reactor operation, where there is potential for a fission product release. An example of this is a source term accident for a high temperature gas-cooled reactor (HTGR), given in Figure 3. In this situation, nuclear engineers need to be able to understand and predict the fission product behavior as it interacts with different parts of the system. The first issue that must be addressed is how the fission product diffuses through the fuel kernel and the surrounding graphite. Another major concern is how the fission product releases into the reactor 
coolant and the resulting plateout effects. Lastly, effort must be given to understand and predict how the fission product will interact with moisture and dust already present in the environment. This last component of fission product analysis is what is relevant to the research presented in this dissertation.

\section{Aerosol Transport Equation}

To accurately model aerosol particles, the general aerosol transport equation is used. This equation is both nonlinear and integro-differential in nature, making it exceedingly complex ${ }^{1,43}$. For a given number of particle species (components) $N$, assumed well mixed, the general equation takes the form shown in Equation 1:

$$
\begin{aligned}
& \frac{\partial}{\partial t} n(\boldsymbol{v}, \boldsymbol{m}, t)+R(\boldsymbol{v}, \boldsymbol{m}, t) n(\boldsymbol{v}, \boldsymbol{m}, t)+\sum_{p=1}^{N} \frac{\partial}{\partial v_{p}}\left[I_{p}(\boldsymbol{v}, \boldsymbol{m}, t) n(\boldsymbol{v}, \boldsymbol{m}, t)\right]= \\
& \frac{1}{2} \int_{0}^{\infty} d \boldsymbol{u} \int_{0}^{\infty} d \boldsymbol{w} \int_{0}^{\infty} d \boldsymbol{q} \int_{0}^{\infty} d \boldsymbol{s} n(\boldsymbol{u}, \boldsymbol{q}, t) n(\boldsymbol{w}, \boldsymbol{s}, t) K(u, q \mid w, s) \\
& \prod_{p=1}^{N} \delta\left(v_{p}-u_{p}-w_{p}\right) \delta\left(m_{p}-q_{p}-s_{p}\right) \\
& -n(\boldsymbol{v}, \boldsymbol{m}, t) \int_{0}^{\infty} d \boldsymbol{u} \int_{0}^{\infty} d \boldsymbol{q} K(u, q \mid v, m) n(\boldsymbol{u}, \boldsymbol{q}, t)+S(\boldsymbol{v}, \boldsymbol{m}, t)
\end{aligned}
$$

where

$$
n(\boldsymbol{v}, \boldsymbol{m}, t)=n\left(v_{1}, v_{2}, \ldots, v_{N} ; m_{1}, m_{2}, \ldots, m_{N}, t\right)
$$

is the aerosol distribution function and is dependent on species volume, species mass, and time. The aerosol transport equation is generally solved for $n(\boldsymbol{v}, \boldsymbol{m}, t)$. To solve the equation, an initial condition of the form $n(\boldsymbol{v}, \boldsymbol{m}, 0)$ must be provided. Additionally, functions for the deposition rate $R(\boldsymbol{v}, \boldsymbol{m}, t)$, condensation rate $I_{P}(\boldsymbol{v}, \boldsymbol{m}, t)$, coagulation rate $K(u, q \mid w, s)$, and source term $S(\boldsymbol{v}, \boldsymbol{m}, t)$ are considered already known.

By analyzing Equation 1, it is relatively straightforward to establish what each term in the equation represents. The first term on the left hand side of the equation is the 
partial derivative of the aerosol distribution function with respect to time. Term two describes aerosol particles removed from the aerosol. This can be due to deposition, gravitational settling, and/or diffusive means. The third term is to account for particle growth by condensation. It is dependent on the specified condensation growth rate for each species $p$.

On the right hand side of the equation, term one represents the generation of new aerosol particles from coagulation. The " $1 / 2$ " at the beginning of the term is due to the fact that, for each pair of particles that coagulate, only one new particle is created. Within the term reside two delta functions, which ensure that particle volume and particle mass are conserved. The second term describes the aerosol particles lost due to a coagulation event. Term three accounts for an independent source of aerosol particles injected into the preexisting aerosol system.

One thing worth noting is that the general aerosol transport equation given in Equation 1 is spatially homogeneous. This is evidenced by the lack of any coordinate system in the formula. Making the formula spatially inhomogeneous by incorporating a coordinate system would dramatically increase the equation's complexity, causing the expression to become even more difficult to solve.

\section{Aerosol Modeling}

Due to the complexity of the general aerosol transport formula, the equation is commonly solved by using computational modeling and by making approximations. The current standard for aerosol modeling is a program called MAEROS, which was developed at Sandia National Laboratory. This program is a self-contained multicomponent aerosol module that can be found in both the CONTAIN and MELCOR 
modeling codes. In order to solve the aerosol transport equation, MAEROS uses a sectional technique. Unfortunately however, the program is far from optimized. This is because MAEROS has numerous restrictions that puts a limit on the complexity level it is able to solve.

For example, the MAEROS program puts limits on both the number of sections that can be used (20 or less) and the number of species a particle can have (eight or less). When plotting, the user is limited to no more than 50 rows and 101 columns ${ }^{44}$. While these values are usually more than sufficient, working with a particularly complex aerosol problem could potentially require using values greater than the limits imposed by the MAEROS code.

Of greater concern is the restrictions imposed on the deposition, coagulation, and condensation processes. This is because the phenomena that are considered to affect these processes are limited. For example, the deposition process is limited to the effects of gravitational settling, diffusion, and thermophoresis. Similarly, the coagulation process is limited to the effects of Brownian motion, gravity, and turbulence. Condensation is also limited, and only affected by gas condensation (usually as water vapor) and time dependent particle sources ${ }^{44}$. These restrictions potentially limit the user from applying all phenomena relevant to their computations.

Unfortunately, even the allowed phenomena are not without their own restrictions and assumptions. One example of this is the chemical composition of the particle, which is not considered in any of the processes mentioned above. Another is that all the species in a multi-species aerosol are assumed to have the same density ${ }^{10,45-47}$. Both of these examples are non-physical, and as such limit the accuracy of the MAEROS results. 
In order to avoid these restrictions, alternative modeling techniques must be developed. One promising alternative is the DSMC method, which allows the user to successfully incorporate many aspects that were limited in the MAEROS code. The general DSMC approach traditionally follows four steps:

1. Particles are randomly selected from an initial distribution. This distribution is usually in terms of particle mass or volume.

2. A time step $(\Delta t)$ is chosen such that its duration is smaller than the characteristic time length of the fastest aerosol process.

3. Deposition, coagulation, and condensation processes are applied to the particles. Due to the short duration of $\Delta t$, it can be assumed that a given particle only undergoes one process in the given time period.

4. The simulation time is advanced by $\Delta t$, and the simulation repeats the previous step (step 3).

It is important to note that the DSMC technique described here is not of the same complexity as Monte Carlo neutron modeling. Monte Carlo neutron modeling is only concerned with neutron-nuclei interactions, which makes Equation 1 linear. By contrast, the DSMC technique for aerosol particles requires accounting for coagulation interactions, which leads to nonlinearity and a higher degree of complexity.

For this research, the software program Wolfram Mathematica was used to develop a DSMC code that would accurately model aerosol behavior, as well as provide resulting data analysis. Wolfram Mathematica was developed by Wolfram Research, and is a symbolic mathematic computation program. It allows the user to conduct complex symbolic, numerical, and graphical calculations. The program consists of a kernel (which 
interprets input and returns results) and a graphical user interface (GUI). Wolfram

Mathematica was chosen because of numerous features advantageous to the study. These include symbolic representation, ease of list manipulation, built in data visualization tools, and clustering algorithms.

\section{Scope and Organization of Research}

The scope of the research conducted in this dissertation was to further demonstrate the validity of using the mesh-free DSMC technique to model spatially inhomogeneous aerosols. To accomplish this, the method had to be extended from modeling aerosols inside a symmetrically optimized sphere into non-symmetric and complex geometries. This required generalization of the approach, significantly increasing its complexity. In order to demonstrate that the results from the generalized mesh-free DSMC technique were accurate, they were compared with prior literature and the Finite Element Method (FEM) where appropriate. To increase the modeling capability of the computer code, condensation was incorporated into the program.

The remainder of the dissertation is presented in the following ten chapters. Chapter II focuses on the mesh-free DSMC modeling process. A description of the technique is given, as well as at what point the PAM process is incorporated. The third chapter describes the modeling techniques used for diffusion, deposition, coagulation, and condensation. Chapter IV provides the details of the PAM method used during analysis. This includes a discussion on the clustering methodology and where it fits in the PAM process. The fifth chapter describes the development of the mesh-free DSMC program. It focuses on crucial programming modifications in the areas of symmetry, user friendliness, computational speed, and the incorporation of the condensation model. 
Chapter VI introduces the fundamental and complex vessel geometries modeled during the research. The parameters for each geometry are given. Chapters VII through IX present the simulation results for the geometries described in Chapter VI. These chapters correspond to simulations conducted with an increasing number of aerosol phenomena included (deposition, coagulation, and condensation, respectively). Chapter $\mathrm{X}$ provides a brief summary of the work conducted for this project. Lastly, in Chapter XI conclusions regarding the capabilities of the mesh-free DSMC technique employed for this work are drawn. 


\section{MESH-FREE DSMC MODELING}

Conventionally, when a heterogeneous system (such as an aerosol) is modeled, the computational domain is divided into a number of mesh "cells." During every simulated time step, the particles' positions are adjusted. The particles are then grouped together based on the cell number they reside in ${ }^{37}$, and particle interactions are computed for each group. Unfortunately, this procedure depends strongly on the geometry defining the computational domain, as the mesh cells must remain within the boundary. This strong relationship between the cells and the geometry can quickly become computationally demanding as well as extremely labor intensive.

Unlike the mesh-cell method, which typically keeps the cells stationary throughout simulated time, the approach utilized for this research allows for much more dynamic particle clustering. In particular, a clustering algorithm was used that groups particles based on a single factor - Euclidian distance. Using this technique, after the particles have moved during a simulated time step, they are all reclustered allowing for the groupings to adapt in time. Since the clustering algorithm only utilizes Euclidian distance, there is a much weaker relationship to the geometry defining the computational domain, which makes the approach much more suitable for use in arbitrary geometries. Chapter IV provides a more detailed explanation of this clustering algorithm.

To facilitate understanding of this alternate DSMC-based clustering technique, a generalized outline is provided. The fundamental difference between this method and the more traditional DSMC mesh-cell approach takes place in clustering step 2c. Applying the alternate technique to a given simulation yields the following outline: 
1. For a given initial particle mass distribution, $N$ selected particles are placed in a list. Each particle $p$ has a list entry of the form $\left\{m_{p, 1}, m_{p, 2}, \ldots, m_{p, 3}, x_{p}, y_{p}, z_{p}\right\}$, where $m_{p, 1}, m_{p, 2}, \ldots, m_{p, s}$ symbolize the masses of particle species 1 through $s$, and $x_{p}, y_{p}, z_{p}$ represent the Cartesian coordinates for the given particle in the computational domain. These particle coordinates are selected based on an initial aerosol coordinate distribution.

2. The program advances through a single time step $\Delta t$. During this time, several events occur. They are:

a. Each particle moves through the time step. This is due to many effects, such as gravitational settling, Brownian motion, and gas flows. The "Diffusion Modeling" section of Chapter III provides the details of how this modeling was accomplished.

b. Any particle that encounters the geometric boundary is evaluated for deposition. These particles can either deposit on the surface of the geometric boundary or can return to suspension in the gas. To facilitate the simulation computations, it was assumed for this work that all particles coming into contact with the boundary deposit on the surface. Details of this step are given in the "Deposition Modeling" section of Chapter III.

c. All the particles still in suspension and located in the computational domain are clustered using the Partitioning Around Medoids (PAM) method. This process divides the particles into $n_{C}$ clusters as explained in Chapter IV. 
d. Once the clusters in step $2 \mathrm{c}$ are established, the No Time Counter (NTC) technique is applied to each one to conclude how many particle collisions have happened. Colliding particles determined to have interacted (coagulated) with each other are combined together to form a single particle, following conservation of mass assumptions. The coordinates of the resultant particle is a weighted probability between the two old particles' original coordinates. The "Coagulation Modeling" section of Chapter III provides the details of how this modeling was accomplished.

e. Particles determined not to have coagulated in step $2 \mathrm{~d}$ are subjected to condensation effects. The condensation growth is species specific and simulated using the isothermal Mason model. Details of this step are given in the "Condensation Modeling" section of Chapter III.

f. The changes made during the time step are noted, including particles that may have deposited, coagulated, or grown due to condensation. All particles that have not deposited are placed into a list with their new coordinates and masses (if applicable). Any desired output data is saved in designated file directories.

3. The simulated current time is updated by the duration of time step $\Delta t$. Step 2 is then repeated until the simulated current time equals the desired simulation end time. 


\section{DIFFUSION, DEPOSITION, COAGULATION, AND CONDENSATION MODELING METHODS}

In order to accurately simulate aerosol behavior in the mesh-free DSMC technique discussed in the previous chapter, models had to be developed for the diffusion, deposition, coagulation, and condensation processes. Unlike the other three processes, diffusion gets applied to all the aerosol particles. After diffusion has been calculated, the deposition, coagulation, and condensation processes are applied in sequence. As was discussed previously, due to the short duration of the simulation time step, it can be assumed that a given particle only undergoes one of the latter three processes in the given time period. Details on how these processes were modeled are given below.

\section{Diffusion Modeling}

During each time step, the particles suspended within the geometric boundaries are subjected to Brownian motion. In the DSMC program, this effect is modeled by first calculating the diffusion coefficient for each particle $p$ using the formula shown in Equation 2. This formula is of the form

$$
D_{p}=\frac{k_{B} T}{3 \pi d_{p} \mu} \times C_{C} \quad \text { Eq. } 2
$$

where $k_{B}$ is the Boltzmann's constant, $T$ is the temperature, $C_{c}$ is the Cunningham correction factor, $d_{p}$ is the particle diameter, and $\mu$ is the gas (air) viscosity.

Since the mean displacement of particle $p$ in any given direction $r$ due to Brownian motion can be calculated ${ }^{48}$ by the formula $\Delta r_{p}=\sqrt{2 D_{p} \Delta t}$, it is possible to 
calculate the new coordinates of the particle. To do this, a random sample is taken from each of three normal distributions

$$
\begin{array}{ll}
N\left(x_{\text {old }}, \sqrt{2 D_{p} \Delta t_{\text {Brown }}}\right) & \text { Eq. } 3 \\
N\left(y_{\text {old }}, \sqrt{2 D_{p} \Delta t_{\text {Brown }}}\right) & \text { Eq. } 4
\end{array}
$$

and

$$
N\left(z_{\text {old }}, \sqrt{2 D_{p} \Delta t_{\text {Brown }}}\right) \quad \text { Eq. } 5
$$

in order to determine the distance in which the particle has moved in each direction during the given time step. The new particle coordinates are then analyzed to determine if the particle lies within the computational domain. If the particle's new coordinates are on or outside the geometric boundary then the particle is considered deposited, and the appropriate deposition calculations are made (see the "Deposition Modeling" section of Chapter III). Additionally, the particle is dropped from the DSMC list of particles still in suspension. This prevents it from being used in future calculations.

It is important to note that, in the event the simulation time step $\Delta t$ is considered large, the time step can be subdivided so as to allow for multiple consecutive calculations of particle location. This affects the relationship between the Brownian motion time step $\Delta t_{\text {Brown }}$ and the simulation time step $\Delta t$ such that, when the time step is subdivided $c$ times, $\Delta t_{\text {Brown }}=\Delta t / c$. To facilitate the simulation computations, it is assumed in this work that particle diffusion is solely from the effects of Brownian motion.

\section{Deposition Modeling}

For the research conducted in this paper, it was assumed that all particles coming into contact with the limits of the computational domain deposit on the surface. After 
computing the final particle positions in a given $\Delta t$, the coordinates were run through a geometric surface based algorithm that acted as a binary switch. All the particles flagged as having a final position outside the geometric boundaries were recalculated to determine their point of deposition. This was accomplished by approximating the particle travel as a straight line between the initial point $\left(x_{0}, y_{0}, z_{0}\right)$ and the final point $\left(x_{1}, y_{1}, z_{1}\right)$.

For each particle, the line was described using the parametric equations for a line in three-dimensional space

$$
\begin{array}{ll}
x=x_{0}+t\left(x_{1}-x_{0}\right) & \text { Eq. } 6 \\
y=y_{0}+t\left(y_{1}-y_{0}\right) & \text { Eq. } 7 \\
z=z_{0}+t\left(z_{1}-z_{0}\right) & \text { Eq. } 8
\end{array}
$$

where $x, y$, and $z$ are the coordinates at which the particle encounters the boundary and $t$ is unknown. The equations for the surface of the geometric boundaries were rearranged so that the right-hand-side was equal to zero. Equations 6-8 were then substituted into these boundary formulas. Each one was solved for $t$, with any answers containing an imaginary part being discarded.

To accommodate more complex geometric boundaries, all the values of $t$ containing only real numbers were combined into a list. The $t$ value closest to zero was then selected as the particle's proper deposition site. As an illustration for this necessity, suppose a particle resides inside a torus oriented about the $z$ axis. During $\Delta t$, the particle moves towards the axis of symmetry, staying on the $x y$ plane. Based on the particle's final position, up to three values of $t$ could satisfy the conditions of the surface boundary equation: The first as the particle encounters the initial torus boundary, the second as the particle has traveled past the axis of symmetry and has interacted with the inner torus 
boundary on the far side, and the third as the particle passes through the outermost boundary on the far side.

\section{Coagulation Modeling}

When a coagulation event is determined to have taken place, the two particles $p_{1}$ and $p_{2}$ combine to form a new particle. The new particle follows the principle of conservation of mass, and its location is determined using particle weights. Assuming a spherical shape, the mass of any particle is related to the diameter by $m_{p}=\pi \rho d_{p}^{3} / 6$, where $d_{p}$ is the particle diameter. Rearranging this equation in terms of $d_{p}$ and substituting it into Equation 2 yields another expression for the diffusion coefficient. Relating this expression to the calculation for mean Brownian motion particle displacement reveals that particle movement is proportional to $m_{p}^{-1 / 6}$. For any given coagulation event, the position of the resultant particle can thus be given as a weighted probability such that

$$
W_{1}=\frac{w}{1+w} \quad \text { Eq. } 9
$$

and

$$
W_{2}=\frac{1}{1+w} \quad \text { Eq. } 10
$$

where $w=\left(m_{2} / m_{1}\right)^{-1 / 6}$, and $W_{1}$ and $W_{2}$ are the weighted probabilities for particles one and two, respectively.

Another option for determining the coordinates of the resultant particle is to calculate the location of the center of mass for the two particles that coagulate. Unfortunately, this approach would have a detrimental effect on particle distribution, 
especially for computations with a longer amount of simulated time. In each coagulation pair, the particle residing closer to the surface would effectively be "pulled back" toward the center of the geometry. Over time, the particles would migrate toward the center of the geometry, affecting the particle distribution. In order to maintain the spatial distribution, the weighted probability approach shown in Equations 9 and 10 was used for research computations.

To determine the number of particles in each cluster that potentially coagulate, the NTC method was applied. According to $\operatorname{Bird}^{37}$, the equation for the number of interacting particle pairs in each cluster is

$$
\text { Pairs }=\frac{1}{2} N^{2} \beta \Delta t / V \quad \text { Eq. } 11
$$

where $N$ is the number of particles, $\beta$ is the maximum coagulation kernel, $\Delta t$ is the time step, and $V$ is the cluster volume. It is important to note that $\Delta t$ is deliberately chosen so that the ratio of the time step to the mean collision time per particle is small. For the research presented in this dissertation, the time step was limited so that the number of interacting particle pairs that occur would be less than one percent of the total number of particles in the given cluster. This limited the range of the time step, as well as related it to the number of simulated particles and the aerosol number density in the specified cluster.

It is important to note that the maximum coagulation kernel $\beta$ in Equation 11 consists of contributions from Brownian motion, as well as gravitational and turbulence effects. In equation form this is represented as

$$
\beta=\beta_{\text {Brown }}+\beta_{\text {Grav }}+\beta_{T} \quad \text { Eq. } 12
$$


where $\beta_{\text {Brown }}$ is the Brownian contribution, $\beta_{\text {Grav }}$ is the gravitational contribution, and $\beta_{T}$ is the turbulence contribution. Since the scope of this work limited particles'

displacement to the effects of Brownian motion, $\beta=\beta_{\text {Brown }}$. To determine the maximum coagulation kernel, it therefore becomes necessary to solve the equation

$$
\beta_{\text {Brown }}=\frac{2 \pi}{\varphi}\left(D_{i}+D_{j}\right)\left(\gamma_{i} d_{i}+\gamma_{j} d_{j}\right) \quad \text { Eq. } 13
$$

where

$$
\begin{aligned}
& \varphi=\frac{d_{i}+d_{j}}{d_{i}+d_{j}+2 \sqrt{g_{i}^{2}+g_{j}^{2}}}+\frac{8\left(D_{i}+D_{j}\right)}{\left(d_{i}+d_{j}\right) \sqrt{V_{i}^{2}+V_{j}^{2}}} \\
& g_{i}=\frac{1}{3 D_{i} \ell_{i}}\left[\left(d_{i}+\ell_{i}\right)^{3}-\left(d_{i}^{2}+\ell_{i}^{2}\right)^{3 / 2}\right]-d_{i} \\
& \ell_{i}=\frac{8 d_{i}}{\pi V_{i}} ; \quad V_{i}=\sqrt{\frac{8 k_{B} T}{\pi m_{i}}}
\end{aligned}
$$

In Equation 13, subscripts $i$ and $j$ denote the $i$ th and $j$ th particle. $D$ is the diffusion coefficient, $\gamma$ is the particle shape factor, $d$ is the particle diameter, $k_{B}$ is the Boltzmann's constant, $T$ is the temperature, and $m$ is the particle mass ${ }^{49}$.

\section{Condensation Modeling}

If neither of the previous two processes were applied to a given aerosol particle, the particle was considered to have grown due to condensation. To simulate the growth of each particle, an isothermal Mason model was used. This model assumes spherical particles and ignores the added complications of thermal diffusion and its cross-effects. According to Loyalka and $\operatorname{Park}^{50}$, the condensation rate $\Psi$ for a given aerosol particle under isothermal conditions is 


$$
\Psi=4 \pi R D_{C} \rho_{V}(S-1) f_{C}\left(K n_{C}\right) \quad \text { Eq. } 14
$$

where $R$ is the particle radius, $\rho_{V}$ is the saturation vapor density, and $S$ is a saturation ratio with a value of 1.00374 . The diffusion coefficient for vapor, $D_{C}$, is determined by using the equation

$$
D_{C}=21.2 \times 10^{-6} \times\left(1+0.0071\left(T_{K}-273.15\right)\right) \quad \text { Eq. } 15
$$

where $T_{K}$ is the temperature in Kelvin and is valid over the narrow temperature range $273.15-318.15 \mathrm{~K}^{51}$

To account for Knudsen effects in the condensation model, Equation 14 incorporates the function $f_{C}\left(K n_{C}\right)$. This function takes the form

$$
f_{C}\left(K n_{C}\right)=\left[1+K n_{C}\left(\frac{\sqrt{\pi} K n_{C} \xi_{C} \varsigma_{C}}{K n_{C} \xi_{C}+1}\right)\right]^{-1} \quad \text { Eq. } 16
$$

and

$$
K n_{C}=\frac{2 D_{C}}{R} \sqrt{\frac{m_{V}}{2 k_{B} T_{K}}} \quad \text { Eq. } 17
$$

where $\xi_{C}$ and $\zeta_{C}$ are constants (1.3333 and 1.0161, respectively), $D_{C}$ is the diffusion coefficient for vapor given by Equation $15, m_{V}$ is the mass of the vapor molecule, $k_{B}$ is Boltzmann's constant, and $T_{K}$ is the temperature in Kelvin.

Once the condensation rate of each particle has been determined, the total amount of condensation is computed. This is accomplished by simply multiplying the condensation rate by the time step duration $\Delta t$. The condensation is then added to the preexisting amount of mass for that particle. 


\section{THE PAM TECHNIQUE}

Having established the methods by which diffusion, deposition, coagulation, and condensation take place, the next step required determining how to accurately represent clusters of particles which are allowed to interact with each other. In order to do this for spatially inhomogeneous aerosols, it was necessary to abandon the more traditional DSMC approach wherein the computational domain is divided into a number of fixed cells (a mesh). Instead, a mesh-free alternative is used that takes advantage of the Partitioning Around Medoids (PAM) process, a technique that incorporates Euclidian distance in its clustering algorithm. To determine the appropriate number of clusters, a clustering methodology was developed first. This methodology was then applied to the PAM process.

\section{Clustering Methodology}

The primary difference between the PAM approach to DSMC and the more traditional mesh-based method lies in how the two approach clustering groups of particles. As shown in Figure 4, the clustering algorithm creates cluster boundaries grouped tightly around the outermost particles. In the PAM technique for three dimensions, the boundaries of each cluster are produced by the formation of a convex hull around the outermost points. These clusters are formed by medoid selection and the calculation of the Euclidian distance for each particle. Details of this technique are discussed later.

Unfortunately, as can readily be seen in the two-dimensional example of Figure 4, the clustering method being utilized does not encompass the entire computational 
domain. This is due to the PAM method's dependence on medoid selection and particle scatter to create cluster boundaries, and must be resolved because of the direct affect it will have on the NTC method. If left unaddressed, the coagulation kernel to cluster volume ratio will go up when performing the particle pair computation (Equation 11), resulting in an artificially high number of interacting particle pairs. To overcome this obstacle, each cluster's volume must be scaled so that their sum equals the same volume as the entire computational domain. This eliminates "volume loss" and maintains the accuracy of the collected data.

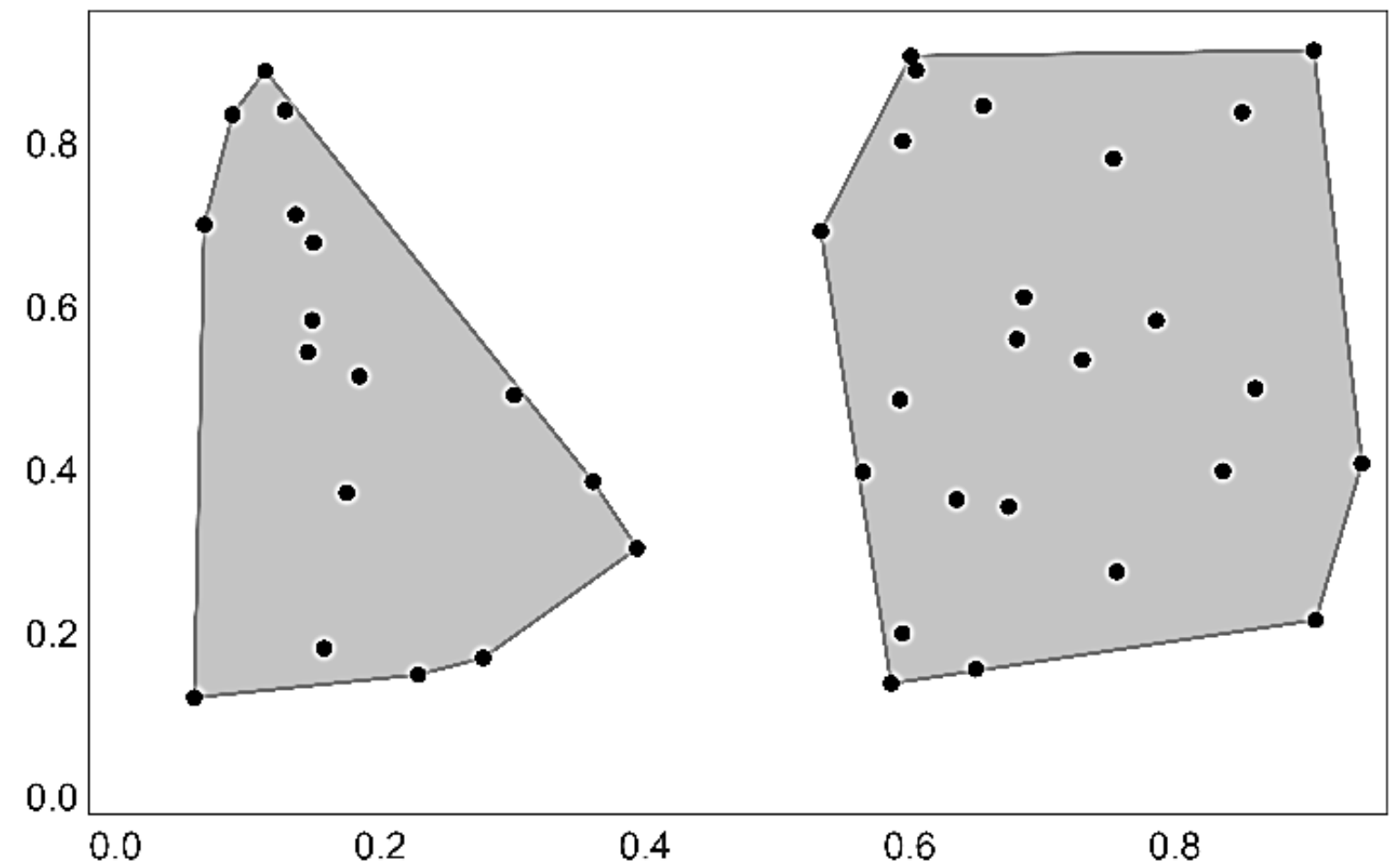

Figure 4: Clustering Algorithm in 2D

To illustrate the difference between the PAM approach to DSMC and the more traditional mesh-based method, the same data points given in Figure 4 are used again for a two-dimensional mesh in Figure 5. For this technique, only particles in the same mesh cell are allowed to interact. The problem this limitation creates can be clearly seen in the 
upper-right corner of the figure, where particles in close proximity to each other have been divided by the mesh boundary. Due to this division, the particles are not allowed to interact with each other. However, these same particles are allowed to interact with others in their same mesh cell near the origin, a distance much further away.

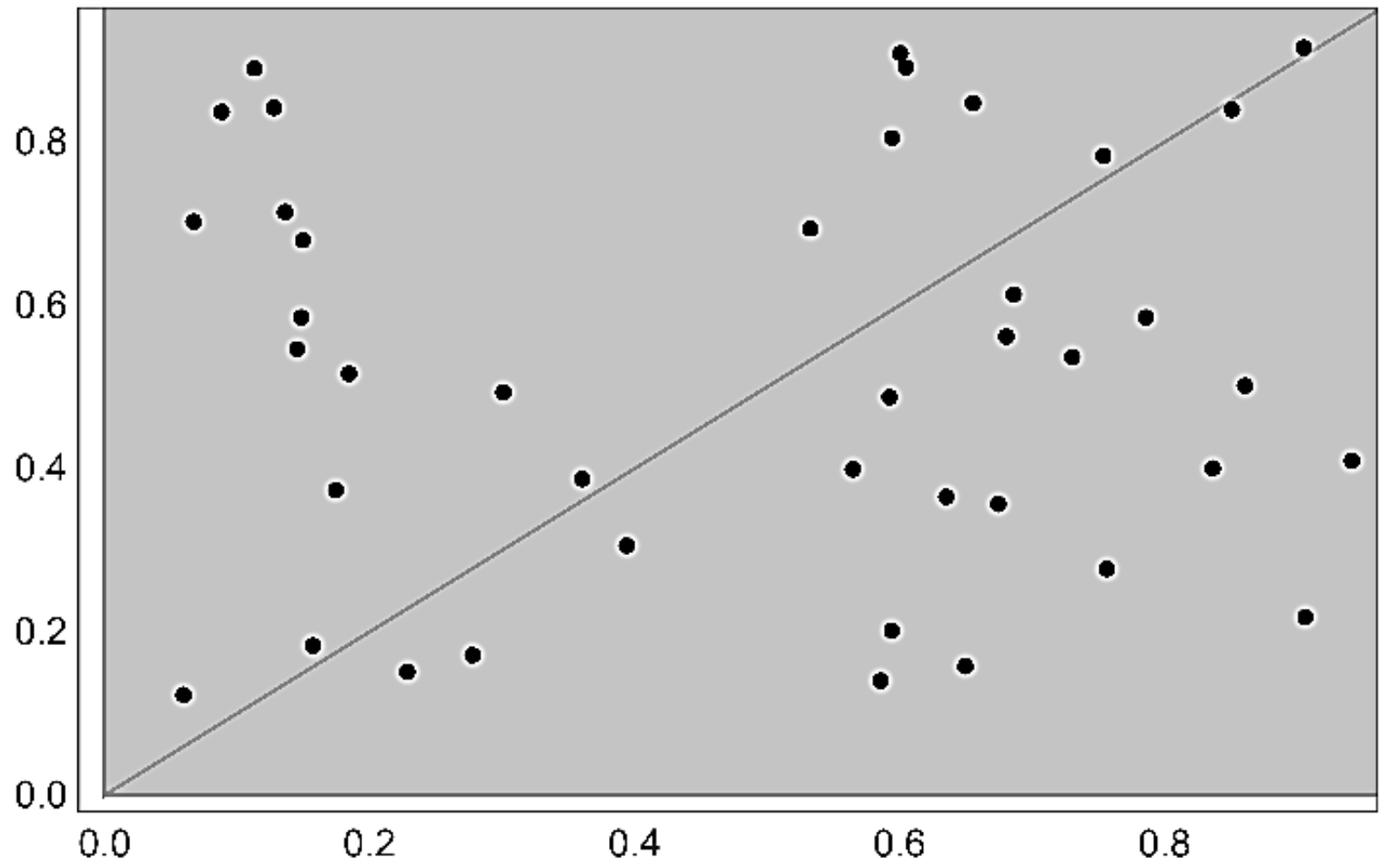

Figure 5: Arbitrary Mesh in 2D

The reason the traditional mesh-cell DSMC method continues to be used is because, when it is utilized to make calculations, there is a tremendous amount of control that is retained over the placement of the mesh. This control even extends to include the parameters for each cell. As a result, the cell boundaries are usually chosen so as to take advantage of aerosol flow and minimize the number of associated properties that need to be recorded ${ }^{36}$.

Regrettably, the PAM technique does not have this high degree of control. This is due to the instantaneous nature of the program: During each $\Delta t$, the particles are 
clustered based on their relative positions. For the next time step, the particles are reclustered based upon their new coordinates. Due to this factor, the primary way to control the clusters is to dictate the number of clusters being used in the computations.

With a thoughtful selection of the number of clusters $n_{C}$ used in the simulation, a moderate amount of authority can be gained over the clusters and the aerosol particles inside each one.

Fortunately there are numerous ways to select the number of clusters to be used in a given calculation, all of which are dependent on a unique combination of simulation parameters. For the research presented in this dissertation, the cluster calculation rule of thumb mentioned by Mardia, Kent, and Bibby was utilized ${ }^{52}$. This rule of thumb states that the number of clusters to use for a given simulation is approximately equal to the square root of the number of particles $N$ divided by two. In equation form, this is represented by:

$$
n_{C}=\sqrt{N / 2} \quad \text { Eq. } 18
$$

To make a more simulation-specific computation on the required number of clusters, another approximation was developed. Unlike the simplistic form of Equation 18, which depends solely on the total number of simulated particles in the given time step, the revised approximation bases its calculation on three different parameters. These three parameters are the duration of the time step, the volume of the computational domain, and the particles' mobility. In particular, the development of this alternate approximation seized on the fact that, for a given cluster, the ratio of cluster particles crossing the boundaries over the total number of cluster particles needs to be small. The small ratio is necessary so that it can be safely assumed that the particles in a given 
cluster only interact with each other. This is an assumption that is critical to the validity of the clustering approach.

Due to this assumption, the number of clusters $n_{C}$ chosen for this approximation was linked to the mean displacement $\Delta x$ of the particles inside the geometric boundaries. Keeping the safety factor determined by a previous study, the cluster diameter $d_{C}$ was defined to be 100 times the length $\Delta x^{33}$. If the clusters are approximated as spherical, the resulting equation for $n_{C}$ can be given as

$$
n_{C}=\frac{6 V}{\pi(100 \times \Delta x)^{3}} \quad \text { Eq. } 19
$$

where $V$ is the volume of the computational domain and $\Delta x$ is the mean displacement for an average particle during the time step $\Delta t$. The particles' displacement was limited to the effects of Brownian motion for the research presented in this dissertation. Applying the formula for the mean particle displacement under Brownian motion to Equation 19, the equation can be rewritten to provide another rule of thumb:

$$
n_{C}=\frac{6 V}{\pi\left(100 \sqrt{2 D_{p} \Delta t}\right)^{3}} \quad \text { Eq. } 20
$$

Unlike the previous cluster calculation, Equation 20 includes the diffusion coefficient as one of the formula parameters. This indicates that the calculation is closely related to particle mobility. One example of this dependence is given by Campbell et al. in the case of a sphere with a radius of $0.5 \mathrm{~cm}$. For particles ranging from $0.001 \mu \mathrm{m}$ to $0.01 \mu \mathrm{m}$ in a free molecular regime environment, it was determined that only one cluster was appropriate ${ }^{33}$. In the PAM-based DSMC aerosol model, both Equation 18 and 
Equation 20 are calculated, with the value of $n_{C}$ being assigned to the lesser value of the two equations.

Note that, if a given cluster has a size that is too large, permitting any two particles within the cluster to interact becomes a poor approximation of reality. This is because the particles chosen to collide could come from opposite ends of the cluster, a distance now prohibitive for an actual interaction. Allowing such a collision to take place would introduce a particle distribution error into the geometric shape, with the potential for the error to compound over successive time steps. A solution to this was suggested by Bird, who recommends solving the issue for a generalized mesh by dividing each cell by a submesh ${ }^{37}$. Each submesh cell would then be analyzed for particle interaction, guaranteeing that only neighboring particles are allowed to interact with each other. Applying such a method to this work remains an area of potential future exploration. Additional exploration can also be conducted into alternate methods of determining appropriate values for $n_{C}$.

\section{The PAM Algorithm}

With the appropriate value of $n_{C}$ determined from Equation 18 and Equation 20, the PAM algorithm can be employed. As mentioned previously, this method was chosen because of its common use for machine learning and group theory purposes, as well as its speed. A detailed description of the PAM technique will not be provided in this dissertation, and it is recommended that individuals unfamiliar with the method refer to other literature ${ }^{38,39}$. However, a brief description, necessary for understanding the presented research, is given below. 
The goal of the PAM method is to group particles into clusters by minimizing the difference between medoids and the surrounding particles. As the algorithm starts, it accepts the list of particle coordinates and the number of clusters $n_{C}$ to be used.

Calculation of Euclidian distance, the tool used for evaluating which cluster a particle resides in, is already incorporated into the program.

Once the program has received the input, the algorithm enters the first of two phases - the build phase. In this phase, $n_{C}$ particles are selected at random to serve as initial medoids. The distances from these medoids to the particles are calculated, with each particle being assigned to the nearest medoid. This creates an initial group of $n_{C}$ clusters containing all the given particles, with each particle grouped by medoid proximity.

When the PAM code finishes the build phase, it enters the swap phase. During this phase, the program attempts to improve the clustering by ascertaining if some other combination of medoids would decrease the total error - that is, the sum of the medoidparticle distance for each point in a given cluster. For a given cluster under evaluation, the PAM algorithm first calculates the mean medoid-particle distance $\bar{D}_{m}$. Next, the code recursively "swaps" a particle $i$ from the cluster to serve as a potential medoid and determines the average distance $\bar{D}_{i}$ from the particles in the cluster to that point. If $\bar{D}_{i}<$ $\bar{D}_{m}$, then particle $i$ is used as the new medoid for the cluster. Because the location of a medoid has changed, the entire geometry gets reclustered, and the process repeats. Once the optimum arrangement of medoids has been found, the program terminates and the final clusters are outputted. 
For clarity, an example of the PAM method is illustrated with 15 particles being divided into two clusters (see Figure 6 and Figure 7). The end of the build phase is shown in Figure 6. In this phase, two particles have been selected at random to serve as medoids and the Euclidean distance to each particle has been calculated. By associating each particle with its nearest medoid, the particles have been successfully clustered. Note that, in order to aid understanding, artificial lines have been introduced to help associate the particles with their respective medoid.

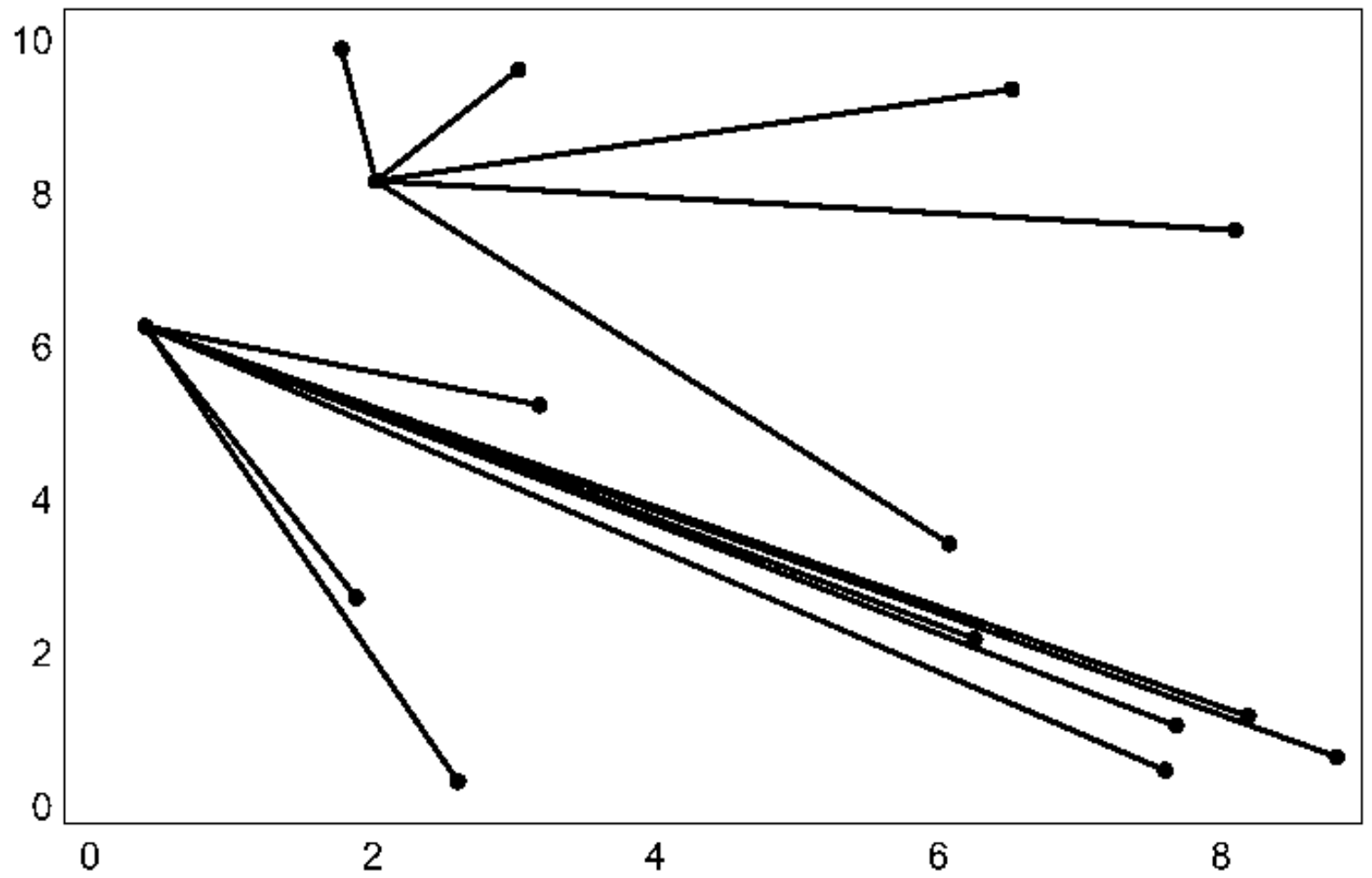

Figure 6: PAM Medoid Selection, Initial Attempt

At this point, the program has yet to find medoids that minimize the overall distance to the particles, as this is done in the swap phase. During this phase, other particles are randomly selected to act as medoids to determine if the overall medoidparticle distance can be reduced. This recursive particle selection process continues until the overall distance is minimized, at which point the algorithm terminates and the final 
clusters are outputted. One such selection is given in Figure 7, with the decrease in overall distance readily apparent. Medoid-particle distances for both the initial and revised selection attempts are shown in Table 1. In this table, the minimum medoidparticle distances for each case are summed to obtain the overall distance for each attempt. Note that the revised attempt has less than half the overall distance of the initial attempt.

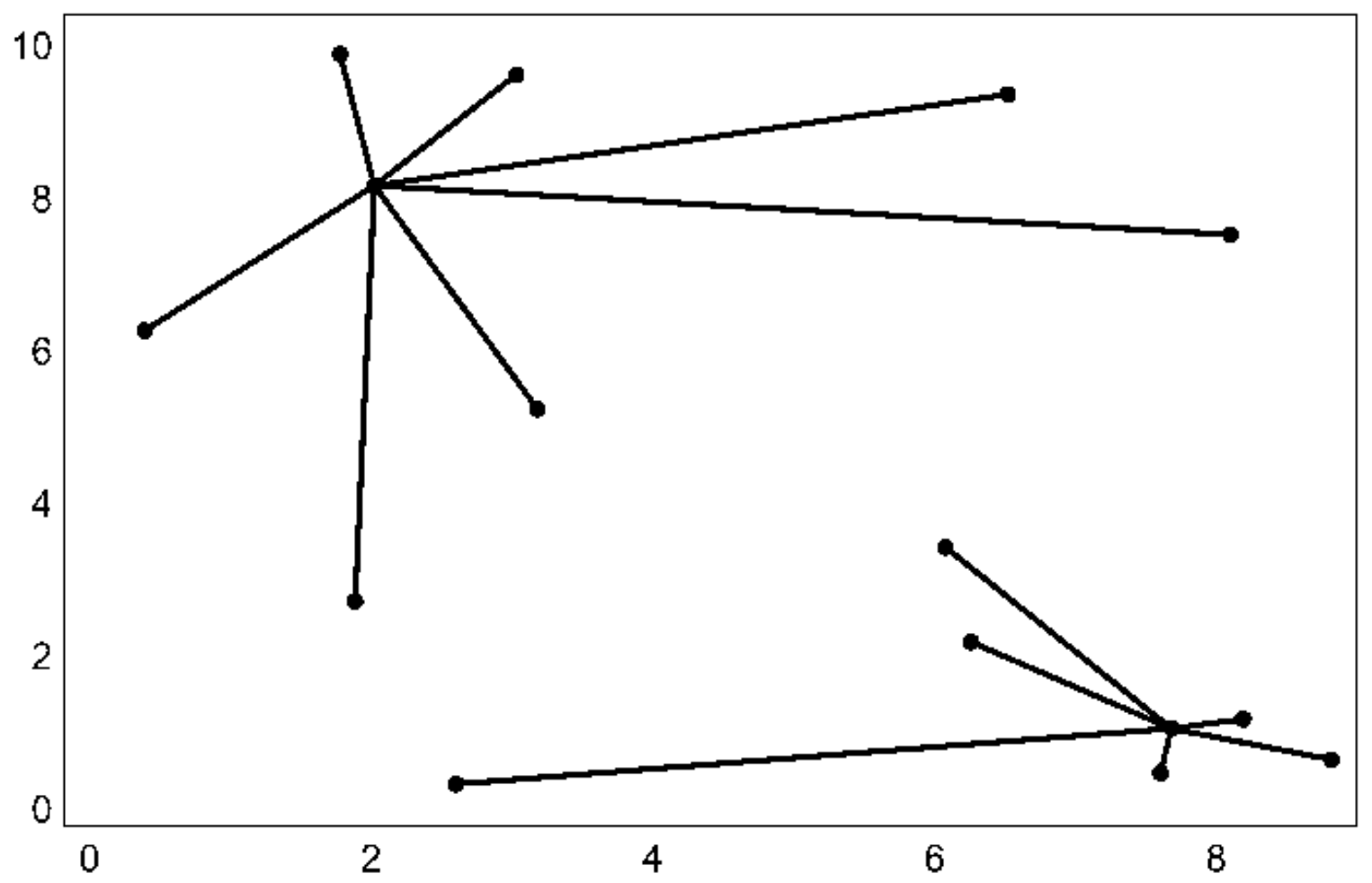

\section{Figure 7: PAM Medoid Selection, Revised Attempt}

While the PAM algorithm described above worked well for this research, alternative approaches to the method exist. One such alteration could include providing an initial list of medoids instead of letting the program select them at random. This would allow the program to work off a partially optimized initial medoid list, potentially cutting computational time. Another alteration would be to limit the number of recursions during the "swap" phase of the program. Such a restriction could potentially affect cluster 
quality, but it would also enforce a computational time limit on the PAM algorithm.

Additional approaches to PAM, such as the ones described here, remain an area of potential future exploration.

Table 1: Total Distance Calculations for Initial and Revised Attempts

\begin{tabular}{|c|c|c|c|c|c|c|}
\hline & \multicolumn{3}{|c|}{ Initial Medoid Calculation } & \multicolumn{3}{|c|}{ Revised Medoid Calculation } \\
\hline $\begin{array}{c}\text { Particle } \\
\text { Coordinate: }\end{array}$ & $\begin{array}{l}\text { Medoid A } \\
\text { Distance: }\end{array}$ & $\begin{array}{l}\text { Medoid B } \\
\text { Distance: }\end{array}$ & $\begin{array}{l}\text { Minimum } \\
\text { Distance: }\end{array}$ & $\begin{array}{l}\text { Medoid A } \\
\text { Distance: }\end{array}$ & $\begin{array}{l}\text { Medoid B } \\
\text { Distance: }\end{array}$ & $\begin{array}{l}\text { Minimum } \\
\text { Distance: }\end{array}$ \\
\hline$(1.88,2.73)$ & 5.45 & 3.85 & 3.85 & 5.45 & 6.03 & 5.45 \\
\hline$(2.60,0.34)$ & 7.86 & 6.34 & 6.34 & 7.86 & 5.13 & 5.13 \\
\hline$(1.78,9.90)$ & 1.74 & 3.88 & 1.74 & 1.74 & 10.63 & 1.74 \\
\hline$(2.02,8.18)$ & 0.00 & 2.50 & 0.00 & 0.00 & 9.09 & 0.00 \\
\hline$(0.39,6.28)$ & 2.50 & 0.00 & 0.00 & 2.50 & 8.96 & 2.50 \\
\hline$(3.03,9.63)$ & 1.77 & 4.27 & 1.77 & 1.77 & 9.75 & 1.77 \\
\hline$(3.18,5.25)$ & 3.15 & 2.97 & 2.97 & 3.15 & 6.15 & 3.15 \\
\hline$(8.19,1.19)$ & 9.32 & 9.32 & 9.32 & 9.32 & 0.53 & 0.53 \\
\hline$(7.68,1.06)$ & 9.09 & 8.96 & 8.96 & 9.09 & 0.00 & 0.00 \\
\hline$(8.82,0.66)$ & 10.14 & 10.13 & 10.13 & 10.14 & 1.21 & 1.21 \\
\hline$(6.08,3.44)$ & 6.24 & 6.36 & 6.24 & 6.24 & 2.86 & 2.86 \\
\hline$(6.26,2.20)$ & 7.33 & 7.15 & 7.15 & 7.33 & 1.82 & 1.82 \\
\hline$(6.52,9.37)$ & 4.65 & 6.87 & 4.65 & 4.65 & 8.39 & 4.65 \\
\hline$(8.10,7.54)$ & 6.11 & 7.81 & 6.11 & 6.11 & 6.49 & 6.11 \\
\hline$(7.60,0.48)$ & 9.51 & 9.25 & 9.25 & 9.51 & 0.59 & 0.59 \\
\hline & \multicolumn{2}{|r|}{ instate. } & 78.48 & \multicolumn{2}{|c|}{ Total Distance: } & 37.51 \\
\hline
\end{tabular}




\section{PROGRAM DEVELOPMENT AND MODIFICATIONS}

During the development of the mesh-free DSMC aerosol model and the PAM algorithm, several modifications were made to the programming code. These programming changes were necessary to generalize the code and make it applicable to arbitrary vessel geometries. They also were made to increase the model's level of user friendliness and to minimize the total computational time. In order to provide more detail and understanding about the critical modifications that were made, descriptions of these changes are given in the following sections.

\section{Geometry Generalization}

First, a series of modifications were made to the program to generalize the vessel geometry. This was necessary because, in the original program, the code had been optimized to take advantage of spherical symmetry. Generalizing the program's geometry mainly required taking the spherically optimized equations and replacing them with ones in the form of Cartesian coordinates. In addition to these changes, three modifications were made that drastically affected geometry generalization. They were the creation of a function named "inOutDeterminer," the use of parametric lines to determine particle deposition, and developing an alternative to concentric cell data representation.

As the first of these three modifications, the "inOutDeterminer" function was created to allow for the input of arbitrary geometries. It serves to define the geometric boundaries of the vessel, and as such is critical to successful geometry generalization. To define the vessel boundaries, the "inOutDeterminer" function is given in the form of a surface equation (or series of equations constituting a vessel surface). Pseudocode 
"inOutDeterminer" examples for a sphere and a cuboid are given in Equations 21 and 22 (respectively),

$$
\begin{aligned}
& \text { inOutDeterminer }[x, y, z]=\left(x-x_{0}\right)^{2}+\left(y-y_{0}\right)^{2}+\left(z-z_{0}\right)^{2}-R^{2}<0 \\
& \text { inOutDeterminer }[x, y, z]=\left|x-x_{0}\right|<x_{B n d} \&\left|y-y_{0}\right|<y_{B n d} \&\left|z-z_{0}\right|<z_{B n d}
\end{aligned}
$$

where $\left(x_{0}, y_{0}, z_{0}\right)$ are the offset coordinates, $R$ is the sphere radius, and $\left(x_{B n d}, y_{B n d}, z_{B n d}\right)$ are surface boundary lengths for the cuboid in their respective directions. Note that both Equations 21 and 22 are arranged with relational symbols, specifically the "less than" sign. This form provides a tremendous amount of power, as the "inOutDeterminer" function can be combined with an "If" statement to track particle location.

An example of the power behind this combination can be seen with either of the two equations given above. If the evaluation on the right hand side of the function holds true, the particle location must be inside the boundaries of the vessel. Conversely, if the right hand side of the function evaluates as false, the particle location must be outside the boundaries of the vessel. This means that, as long as vessel boundaries are provided in the form of surface equations, the location of a particle relative to an arbitrary vessel geometry can be determined. Additionally, because terms on the right hand side of the "inOutDeterminer" function can be joined using Boolean operators, unique vessel geometries can be constructed that consist of multiple shapes. The only limit to this process (besides computational time) is whether or not the desired vessel boundaries can be expressed in terms of surface equations.

The second modification that drastically affected program generalization was the incorporation of parametric lines to determine particle deposition location. These lines were necessary because, as was discussed previously, the program uses normal 
distributions (see Equations 3-5) to move the particle throughout the domain. As these distributions are not dependent on the vessel boundaries, the final assigned location of a particle may be outside the vessel itself.

Given this scenario, the program has to determine where on the vessel boundary the particle deposits. In the original program, the code incorporated the radius to take advantage of spherical symmetry. However, this approach does not work in a nonsymmetric geometry. To determine the correct particle deposition sites for nonsymmetric geometries, parametric lines were used. For ease of reference, these equations are given again below and are of the form

$$
\begin{array}{ll}
x=x_{0}+t\left(x_{1}-x_{0}\right) & \text { Eq. } 23 \\
y=y_{0}+t\left(y_{1}-y_{0}\right) & \text { Eq. } 24 \\
z=z_{0}+t\left(z_{1}-z_{0}\right) & \text { Eq. } 25
\end{array}
$$

where $\left(x_{0}, y_{0}, z_{0}\right)$ is the internal point, $\left(x_{1}, y_{1}, z_{1}\right)$ is the external point, $(x, y, z)$ is the deposition site, and $t$ is an unknown value between zero and one.

To determine deposition location, the equations are substituted into the vessel's surface boundary formula for the values of $(x, y, z)$. This formula is then set equal to zero and solved for the $t$ value. For boundary equations resulting in more than one value of $t$, the smallest positive real number (corresponding to the first encountered boundary) is selected. This value is then substituted back into Equations 23-25 to determine the coordinates of the deposition site. To confirm this location, the coordinates are verified by applying the "inOutDeterminer" function. 
Unfortunately, during the incorporation of the particle deposition site modification described above, a problem was encountered. Specifically, it was determined that the $t$ values being returned were not producing coordinates on the surface boundary. Instead, the produced coordinates would yield an extremely tiny negative value when evaluated with the "inOutDeterminer" function. Investigating this issue, it was discovered that the problem was due to machine precision limitations. When the boundary equation is solved for $t$, it has the strong potential to return an irrational value. Due to the infinite number of digits to the right of the decimal place, this value can only be truncated by the computer. If the truncated digits are necessary to calculate coordinates on the vessel surface, the truncation will result in the extremely tiny negative value that was encountered.

In order to solve this problem, the eighth decimal place of each $t$ value was rounded up. This negated the truncation error by producing coordinates with an extremely tiny positive value when evaluated with the "inOutDeterminer" function. Registering this sign change, the program determined it had found a proper deposition site and was able to stop hunting for a solution.

The reason it was determined to round up the eighth decimal place of each $t$ value was because of outcome reliability. Due to the program structure, a data run consisted of 10 simulations, each of which consisted of $10^{5}$ particles $\left(10^{6}\right.$ particles total). On the computer used, modeling these simulations could take upwards of a week. If at any point a single aerosol particle encountered the $t$ value error described previously, the program would abort its computations and the modeling data would be lost. An appropriate decimal place had to be selected so that it would eliminate this issue while 
simultaneously remaining as small as possible. By experiment, this was determined to be the eighth decimal place.

Unlike the previous two generalization modifications, the last modification made to the program was in regards to the presentation of results. In the work by Campbell et al., the sphere is divided into a series of concentric cells and the data is presented graphically as the amount of suspended mass in each cell ${ }^{33}$. Since the research conducted for this dissertation was to extend beyond the symmetric case, an alternative had to be developed. Continuing to use the concentric cell data representation would have affected the calculation of local concentration.

To calculate local concentration in non-symmetric geometries, a replacement approach was developed that utilizes unit spheres. This method is referred to throughout the dissertation as the "Sample Sphere" technique. It selects a predefined number of points along an arbitrary axis and creates unit spheres centered at these points. The local concentration in each sphere is then calculated and can be plotted. In order to maintain compatibility with the work conducted by Campbell et al., this approach was not the only method used for calculations associated with a spherical vessel. Instead, the original concentric cell data representation was also employed for that geometry.

\section{User Friendliness}

Once the modeling program had been generalized, the next step was to make modifications to increase the code's user friendliness. The first big modification was the addition of text-based "binary switches" to enable and disable desired aerosol behaviors. These switches were used to control Brownian motion, deposition, coagulation, condensation, and other effects. Each switch was assigned a True/False value based on 
the parameters of the aerosol simulation that was being conducted, and would trigger numerical assignments later in the program.

As an example, suppose a binary switch was set so that coagulation effects were turned off. This switch would be denoted coagKernel $=$ False . The numerical assignment later in the program would be of the form

$$
\text { const }=I f[\text { coagKernel }=\text { True }, 1,0] \quad \text { Eq. } 26
$$

where the value of const can be either one or zero, depending on the evaluation of coagKernel. The coagulation probability can then be multiplied by this constant, effectively controlling the coagulation phenomenon.

While having numerical assignments like the one in Equation 26 occur later in the program may appear unnecessary, the reason the values weren't assigned directly was because it was deemed less user friendly than expressions such as coagKernel $=$ False . User friendliness concerns were also the reason why a binary $0 / 1$ numerical system was avoided. Another advantage of these text-based switches was that they could be placed in a single designated spot within the program. This created easy code customization by giving the user access to a "master control panel." With this panel, aerosol processes could be quickly turned on and off as needed.

To increase user friendliness even further, a second computational code was created to manage the data output from the main program. Unfortunately, this was necessary because at every designated export time, the main program automatically saved the data in a directory. These data files consist of raw lists of aerosol particles, with each row corresponding to one particle. As mentioned previously, a data run consisting of 10 simulations could easily consist of $10^{6}$ total particles. Combine this with numerous export 
times, and a single data run has the potential to generate hundreds of files with millions of particles, all of which have to be analyzed.

Fortunately, data analysis for millions of particles was what the second computational code was created for. The program imports the data files and compiles them into readable lists. It then processes the data and outputs the requested information. This material is provided in a much more accessible format, such as tables and graphs. Once they are generated, these materials are automatically saved for later use in the same file directory as the original data.

In order to make the required use of the second computational code as seamless as possible, two import-based adjustments were made. First, the data import process was optimized by creating an import module. This module accepts a date (given in numerical format) as input and uses it to locate the correct file directory from which to draw the data files. Second, a few lines of code were added to the original program to export the vessel geometry's offset coordinates as well as the particle weight. This information is then imported directly into the second computational code, reducing the number of parameters the user has to manually enter into the program.

\section{Computational Time}

Unfortunately, due to the modifications made in the previous two sections, the total computational time required to run an aerosol simulation increased significantly. This forfeited speed was necessary in order to incorporate non-symmetric geometries into the program and make it more user friendly. However, in order to minimize the amount of computational speed lost, revisions were made to the code that optimized the programming language commands. These optimizations included taking advantage of 
parallelism, alternate coding structures, and compiling portions of the code into $C$ programming language.

In order to take advantage of parallelism, specialized commands were used in the program. These commands divide the work to be done among the computational cores available on the computer. Once each core completes its calculations, the work is recombined. The advantage to this approach is that, instead of one core doing all the work, the work is evenly distributed. On multicore computers, this can result in a significant decrease in total computational time.

For the research presented in this dissertation, the parallelization commands were usually incorporated into portions of the code that operated on particle lists. This was because repeatedly performing the same function on a large number of particles was deemed an excellent use of the parallelization capability. Since the computer being used had eight cores available, most particle list operations experienced dramatic time decreases.

Sadly however, limitations on parallelization were quickly discovered. The major restriction with the parallelization commands was that they could not be nested. While intuitive, this limitation still created a significant issue for the program. This was because many portions of the code had layered operations, and only one of these layers could be operating in parallel at any given time.

Fortunately, alternate methods were also employed to minimize computational time. One of these methods was the use of a special programming command named "Timing" in order to find the most efficient programming structure. This command, when implemented, returns the result of the evaluation along with the amount of computational 
time it took the computer to determine the answer. By structuring the code in different ways and wrapping it with the "Timing" command, it is possible to determine if an alternate approach returns the same results and is quicker. The more efficient code can then be implemented, and some computational time saved.

Another method that was used to decrease computational time was compiling portions of the program into $C$ programming language. This saved time because, while extremely user friendly, the Wolfram programming language (used by Wolfram Mathematica) operates rather slowly when compared to $C$. Tasks that were considered to be rudimentary were compiled into $C$, run, and then imported back into Wolfram Mathematica. Examples of these tasks include initial aerosol coordinate assignments and determination of initial particle size.

\section{Condensation Model}

The last significant modification to the aerosol modeling program was the incorporation of a condensation model. Unfortunately this was necessary because, in the original program, a condensation model had not been developed. To fix this problem, an isothermal Mason model was developed and tested in an independent self-contained file. This was done to ensure that the model behaved correctly without introducing a source of error into the main code. Details regarding the form of the isothermal Mason model are given in Chapter III.

Once the program was verified, the condensation model was incorporated into the main simulation code. This involved integrating the isothermal Mason model itself, as well as several additional parameters. Just like with the coagulation and deposition effects, condensation was made controllable via a text-based binary switch. Tests were 
then conducted to determine if the addition of the condensation model had been successful.

Unfortunately, these tests demonstrated that an error occurred when both the coagulation and condensation models were used. In the program, the condensation model received all particles and only operated on the ones that did not undergo coagulation. After considerable analysis of the interaction between these two processes in the code, it was determined that the error was due to the coding commands tying the coagulation and condensation processes together.

During this key part of the program, commands were intended to update condensation values within the existing particle list. Instead, they were generating a separate condensation particle list consisting entirely of particles that had undergone the condensation updating procedure. Since some of the particles in the original list coagulated, the list lengths for the original particle list and the condensation particle list were unequal. The unequal lengths of these two lists generated the error messages that were encountered.

When this issue was resolved, another difficulty was encountered. This problem was that, while the spatially inhomogeneous aerosol model successfully outputted data, the data itself was unrealistic. For example, simple mass concentration calculations yielded water droplets with a density of over 32 times the value of pure water. After extensive program analysis, it was determined that the saturation ratio $S$ (used to calculate the condensation rate) was at fault.

The reason for this conclusion was because of the dramatic effect the saturation ratio has on the overall condensation rate (see Equation 14). Originally, this ratio was 
assigned a value of $S=1.00374$, which was determined from previously published literature ${ }^{53}$. However, this value was much too large for the parameters of the aerosol simulation. Instead of using this original value, a much lower condensation ratio of $S=1$ $+10^{-7}$ was incorporated into the program. Once this lower amount was used the spatially inhomogeneous aerosol model produced realistic results. 


\section{VESSEL GEOMETRIES}

Having completed development and modification of the aerosol modeling program, the next step was to determine what vessel geometries were to be used. In order to compare results with preexisting literature, a sphere was chosen as the first shape to be modeled. Additional fundamental vessel geometries that were selected include an ellipsoid, torus, elliptical cylinder, and cuboid. To demonstrate the robustness of the program, a more complex shape was also chosen. This geometry consisted of a sphere with an internal obstruction created by two overlapping miniature spheres. The appendix presents the surface and volumetric equations for these shapes in tabular form, along with notes on equation notation.

\section{Sphere}

As mentioned previously, a sphere was chosen as the first vessel geometry to be incorporated into the program. This shape was selected so that the results could be compared with those in a paper previously published by Campbell et al. ${ }^{33}$ To ensure an accurate comparison of the two aerosol models, the same vessel dimensions provided in the article were used for the current code. By analysis, it was determined that the sphere was to be assigned a diameter of $1 \mathrm{~cm}$, corresponding to a radius $R$ of $0.5 \mathrm{~cm}$. The surface equation for the spherical vessel is

$$
\left(x-x_{0}\right)^{2}+\left(y-y_{0}\right)^{2}+\left(z-z_{0}\right)^{2}-R^{2}=0 \quad \text { Eq. } 27
$$

where $\left(x_{0}, y_{0}, z_{0}\right)$ are the offset coordinates for the geometry. To populate the sphere with an initial list of particles, the "equals" sign in Equation 27 was replaced by a "less than" sign and used as the "inOutDeterminer" function (see Equation 21). Particles with 
coordinates satisfying the surface equation condition were kept, while those not satisfying the surface equation condition were discarded. The vessel, filled with initial aerosol particles, can be seen in Figure 8.

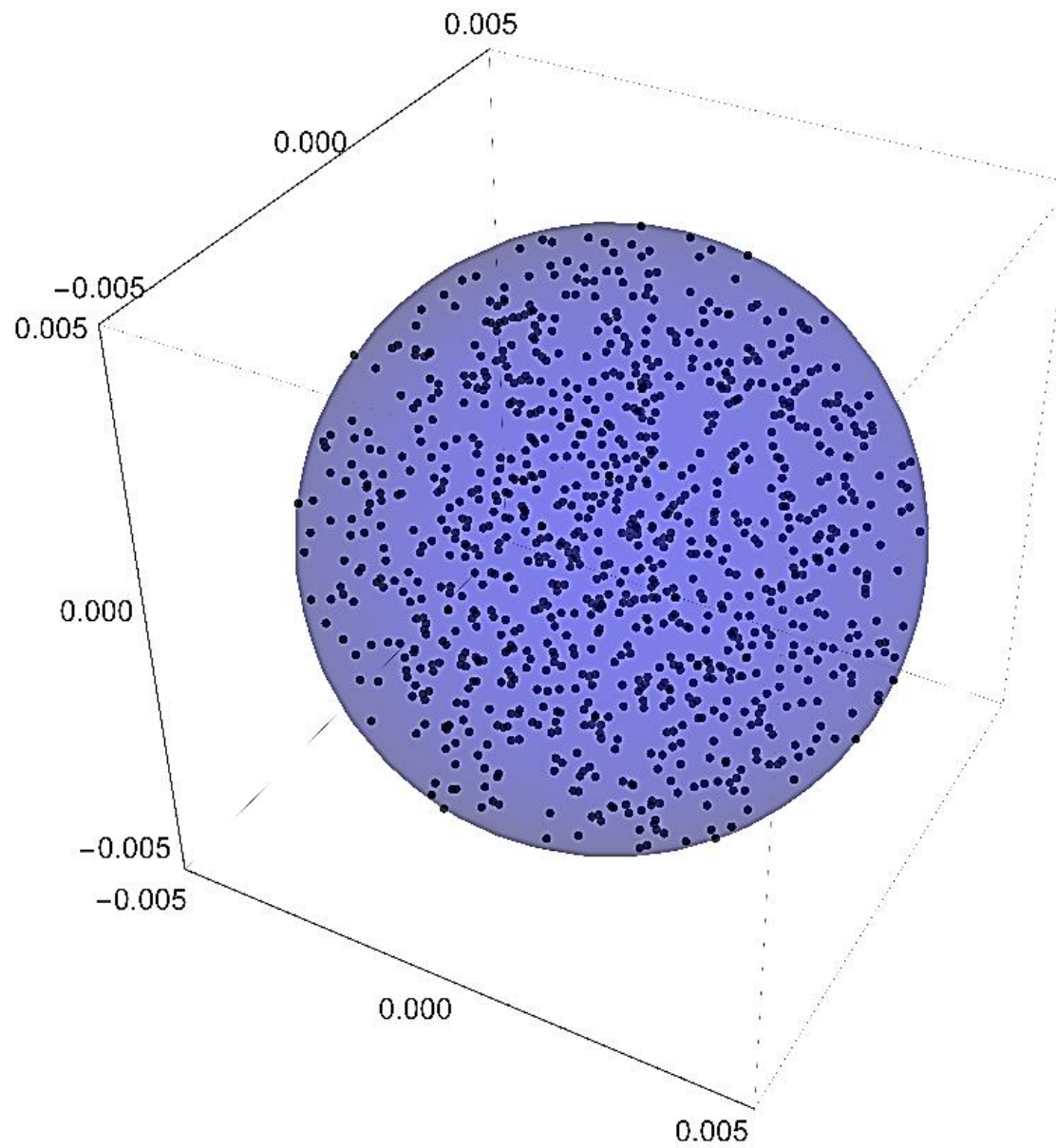

Figure 8: Spherical Vessel with Aerosol Particles

\section{Ellipsoid}

After working with a spherical vessel, an ellipsoid was selected as the next geometry. The reason for this selection was because of the unique relationship between the two shapes - a sphere is simply a special case of an ellipsoid. To maintain the same order of magnitude as the sphere, the axial lengths $(a, b, c)$ in each direction of the ellipsoid were given as fractions of the spherical radius $R$. Specifically, for the geometry 
evaluated, the axial lengths were assigned to be $(R, 0.7 R, 0.5 R)$. The surface equation for the elliptical vessel is

$$
\frac{\left(x-x_{0}\right)^{2}}{a^{2}}+\frac{\left(y-y_{0}\right)^{2}}{b^{2}}+\frac{\left(z-z_{0}\right)^{2}}{c^{2}}-1=0 \quad \text { Eq. } 28
$$

where $\left(x_{0}, y_{0}, z_{0}\right)$ are the offset coordinates for the geometry, and $(a, b, c)$ are the ellipsoid lengths in the $(x, y, z)$ directions, respectively. To populate the ellipsoid with an initial list of particles, the same method used for the sphere was employed. Figure 9 shows the elliptical vessel populated with initial aerosol particles.

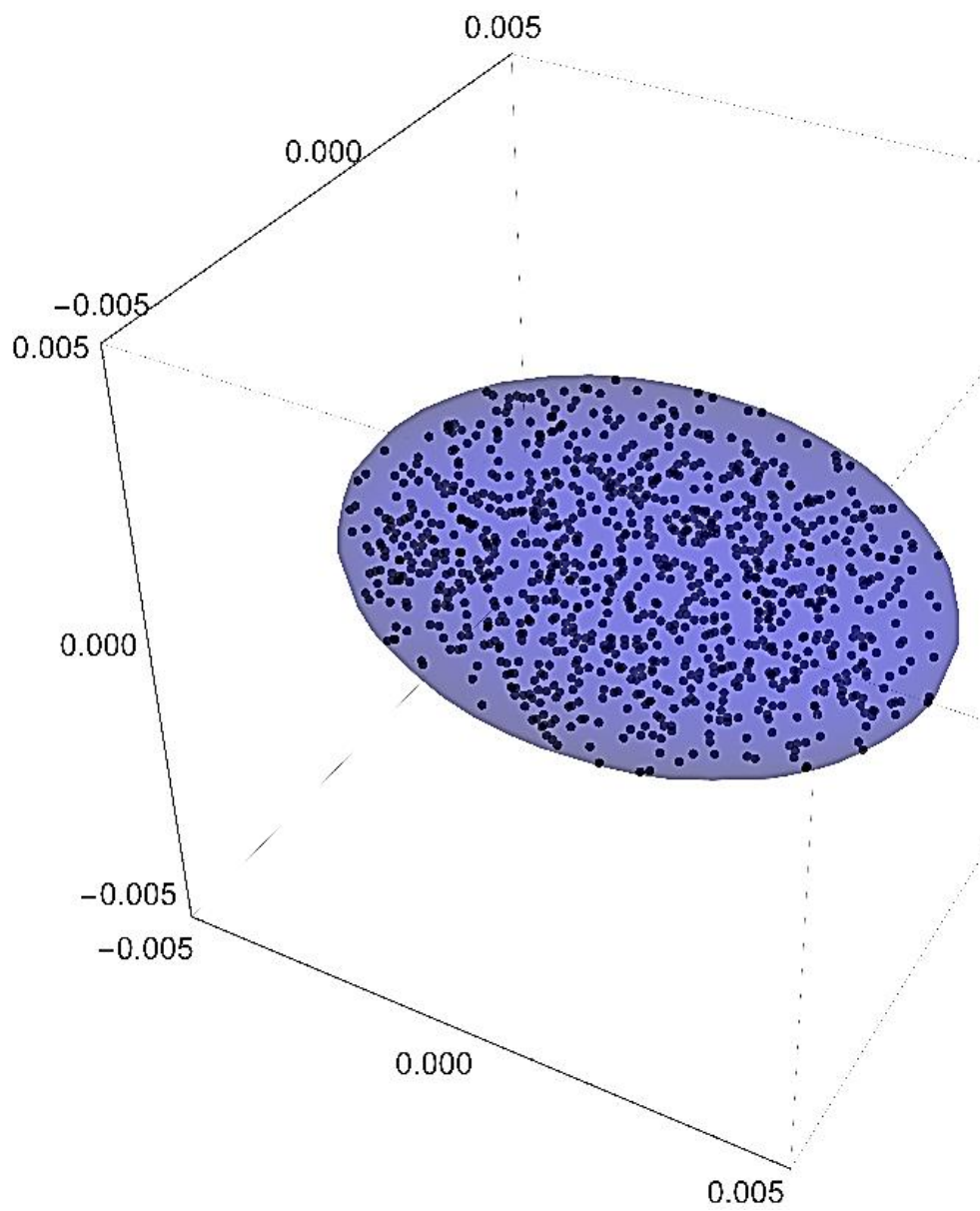

Figure 9: Elliptical Vessel with Aerosol Particles 


\section{Torus}

Once the previous two vessel geometries had been simulated, the next step was to model a torus. This shape was selected for multiple reasons, one of which was having a computational domain that does not encompass the origin (see Figure 10). For the torus, the tube radius $a$ was assigned the same value as the radius of the sphere $(a=R)$, and the distance $c$ from the symmetric axis to the center of the torus tube was assigned the value $c=3 R$. The surface equation for a torus symmetric about the $z$ axis is

$$
\left(c-\sqrt{\left(x-x_{0}\right)^{2}+\left(y-y_{0}\right)^{2}}\right)^{2}+\left(z-z_{0}\right)^{2}=a^{2}
$$

where $\left(x_{0}, y_{0}, z_{0}\right)$ are the offset coordinates for the geometry.

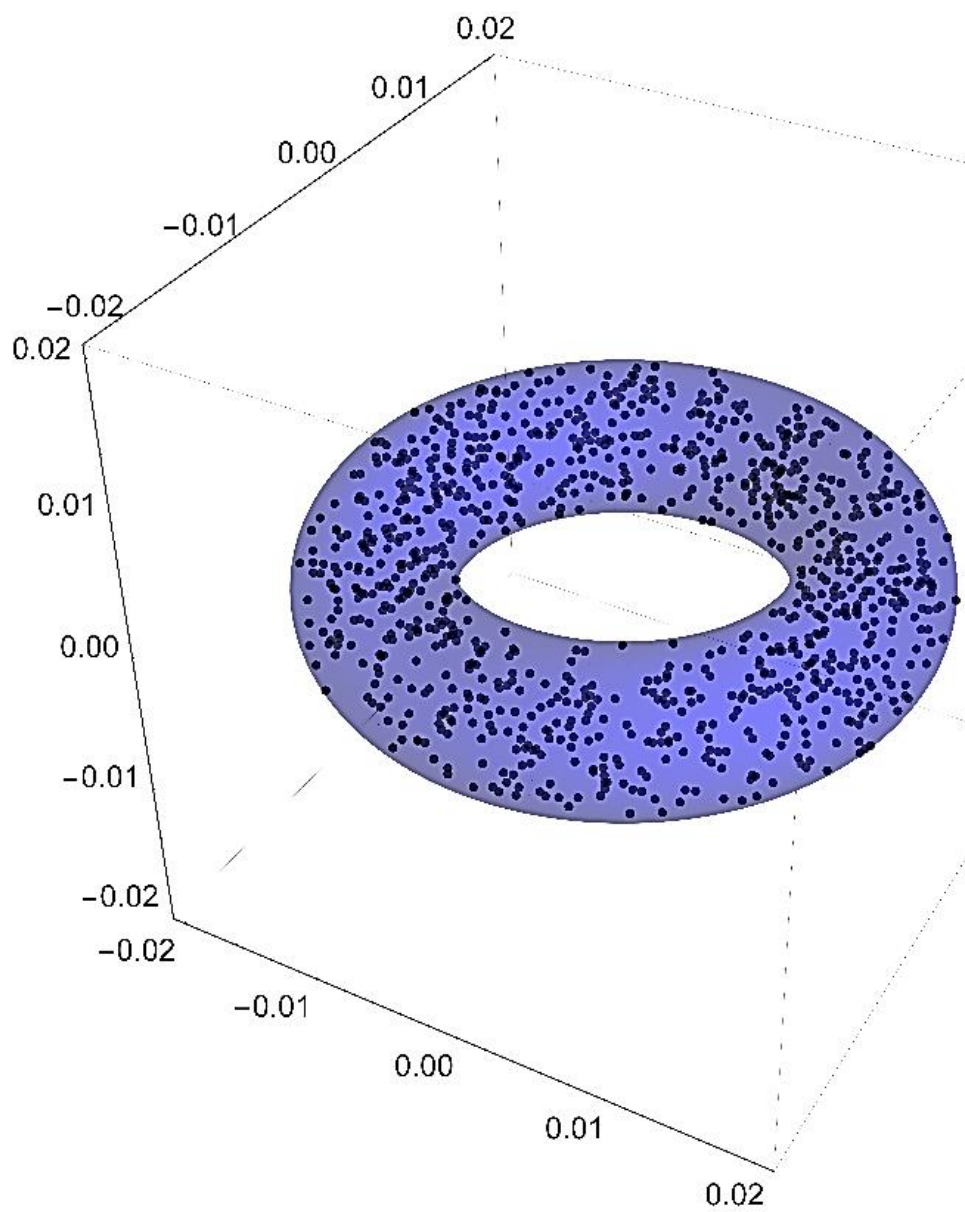

Figure 10: Toroid Vessel with Aerosol Particles 


\section{Elliptical Cylinder}

Having completed computations on a toroid vessel, the geometry was changed to enable the evaluation of aerosol behavior inside an elliptical cylinder. Unlike previous geometries with a single continuous surface, the cylinder provided the opportunity to analyze particle phenomena in a shape with physical discontinuities. The two radii of the ellipse were given values of $a=R$ and $b=0.5 R$, where $R$ is the radius of the original spherical vessel. Additionally, the height $H$ of the cylinder was assigned a value of $H=3 R$. Since this geometry had physical discontinuities, defining the surface of the vessel required more than one surface equation. These equations are

$$
\frac{\left(x-x_{0}\right)^{2}}{a^{2}}+\frac{\left(y-y_{0}\right)^{2}}{b^{2}}-1=0 \quad \text { Eq. } 30
$$

and

$$
\left|z-z_{0}\right|=H / 2 \quad \text { Eq. } 31
$$

where $\left(x_{0}, y_{0}, z_{0}\right)$ are the offset coordinates for the geometry.

To populate the elliptical cylinder with an initial list of particles, the "equals" signs in Equations 30 and 31 were replaced by "less than" signs. These two equations were then joined by a Boolean operator and used in the "inOutDeterminer" function (similar to the cuboid Boolean operator combination given in Equation 22). Due to the nature of the Boolean operator, in order to retain a given particle, its coordinates had to satisfy both conditions. Particles not satisfying the surface equation conditions (or only satisfying one) were discarded. The elliptical cylinder, populated with randomly positioned aerosol particles, is shown in Figure 11. 


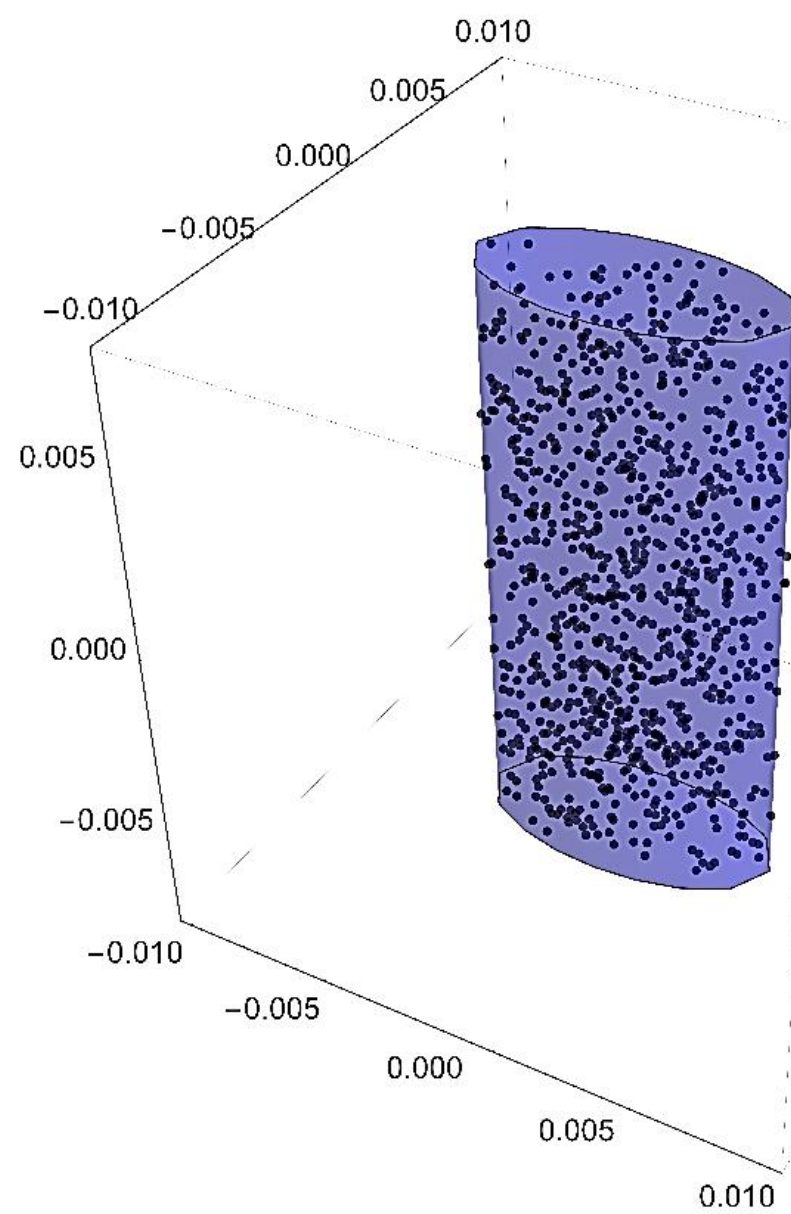

Figure 11: Cylindrical Vessel with Aerosol Particles

\section{Cuboid}

After completing the simulations on an elliptical cylinder, a cuboid was selected as the next geometry. The reason for this selection was because of the development that had occurred on shapes with physical discontinuities. Having successfully modeled a vessel with two surface discontinuities, increasing the number of discontinuities was the next logical step in complexity. Boundary values for the cuboid were of the form $\left(x_{B n d}, y_{B n d}, z_{B n d}\right)$ and assigned to be $(R, R, R)$, where $R$ is $0.5 \mathrm{~cm}$ (radius of the sphere vessel). Note that, although each axis has the same boundary value $R$, these values can be modified to accommodate any cuboid. The surface equations for the cuboid are 


$$
\begin{array}{ll}
\left|x-x_{0}\right|=x_{\text {Bnd }} & \text { Eq. } 32 \\
\left|y-y_{0}\right|=y_{\text {Bnd }} & \text { Eq. } 33
\end{array}
$$

and

$$
\left|z-z_{0}\right|=z_{\text {Bnd }} \quad \text { Eq. } 34
$$

where $\left(x_{0}, y_{0}, z_{0}\right)$ are the offset coordinates for the geometry. Equations 32-34 were then joined by Boolean operators. Figure 12 shows the resultant vessel.

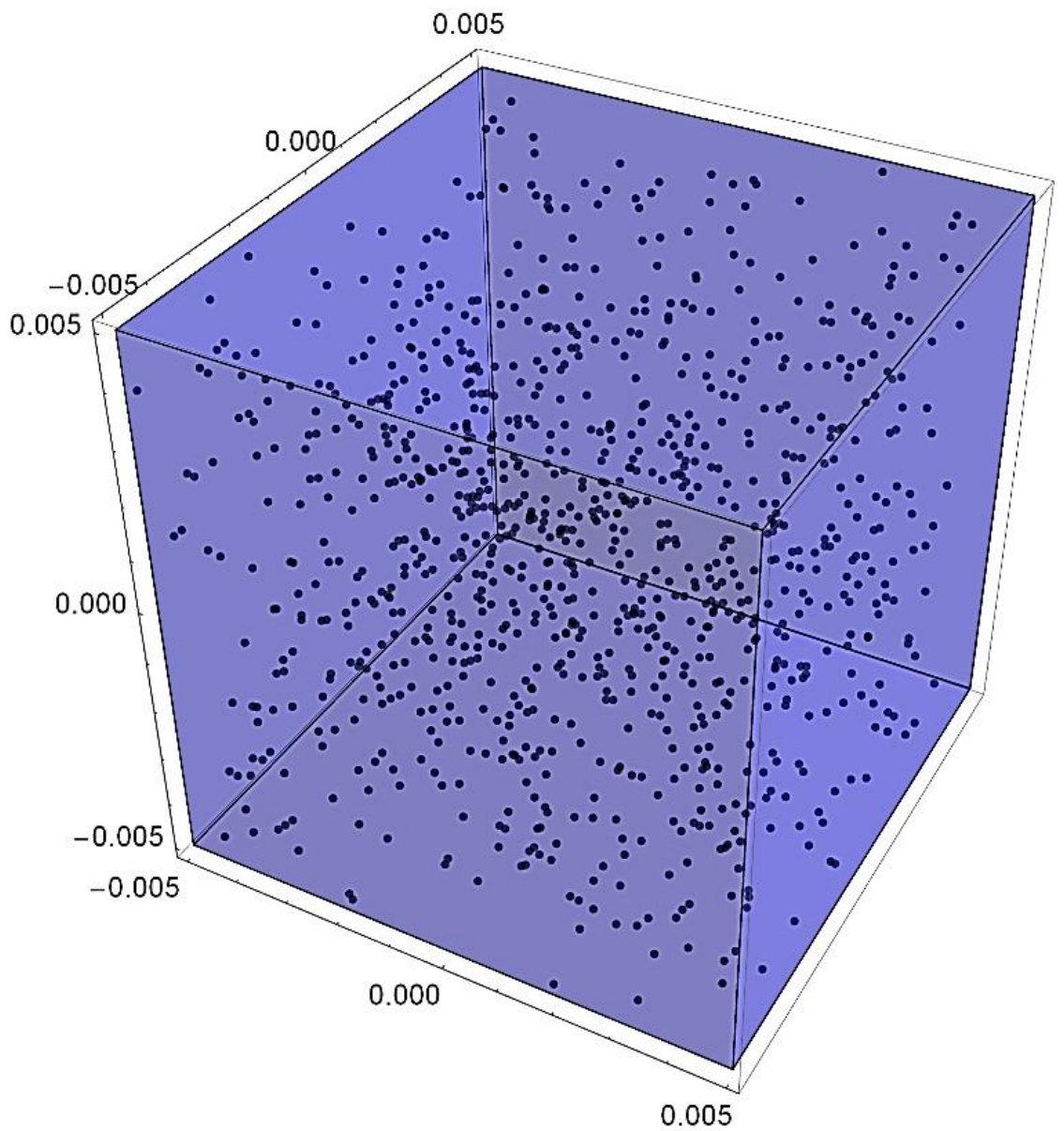

Figure 12: Cuboid Vessel with Aerosol Particles 


\section{Sphere with Internal Obstruction}

Once the program had been run with the fundamental vessel geometries, a more complex shape was developed. This was done to demonstrate the robustness of the program. The complex geometry consisted of a sphere with an internal obstruction created by two overlapping miniature spheres. Aerosol particles were allowed to reside inside the sphere, but not within the internal obstruction. The radius $R$ of the main sphere was assigned the value $0.5 \mathrm{~cm}$ (identical to the original spherical vessel), and the radius of each mini-sphere was assigned to be $R_{\text {obs }}=R / 4$. The offset coordinates for these two mini-spheres were denoted $\left(x_{01}, y_{01}, z_{01}\right)$ and $\left(x_{02}, y_{02}, z_{02}\right)$, and were assigned the values of $\left(-0.5 R_{o b s}, 0,0\right)$ and $\left(0.5 R_{o b s}, 0,0\right)$. The surface equations for this vessel are

$$
\begin{array}{ll}
\left(x-x_{0}\right)^{2}+\left(y-y_{0}\right)^{2}+\left(z-z_{0}\right)^{2}-R^{2}=0 & \text { Eq. } 35 \\
\left(x-x_{01}\right)^{2}+\left(y-y_{01}\right)^{2}+\left(z-z_{01}\right)^{2}-R_{\text {Obs }}{ }^{2}=0 & \text { Eq. } 36
\end{array}
$$

and

$$
\left(x-x_{02}\right)^{2}+\left(y-y_{02}\right)^{2}+\left(z-z_{02}\right)^{2}-R_{O b s}^{2}=0 \quad \text { Eq. } 37
$$

where $\left(x_{0}, y_{0}, z_{0}\right)$ are the offset coordinates for the main sphere.

Just like with all of the previous geometries, this vessel required replacing the "equals" signs in the equations. However, due to the internal obstruction, not all of the "equals" signs in Equations 35-37 were replaced with "less than" symbols. While this was the case for the first equation, the latter two had the "equals" sign replaced with a "greater than" symbol. The surface equations were then combined with with Boolean operators. In order to retain a given particle, its coordinates had to satisfy all conditions. Particles not satisfying the surface equation conditions (or only satisfying one or two) 
were discarded. The spherical geometry, complete with its interior obstruction and randomly positioned aerosol particles, is shown in Figure 13. Note that the aerosol particles lie within the spherical shell but are outside the interior obstruction.

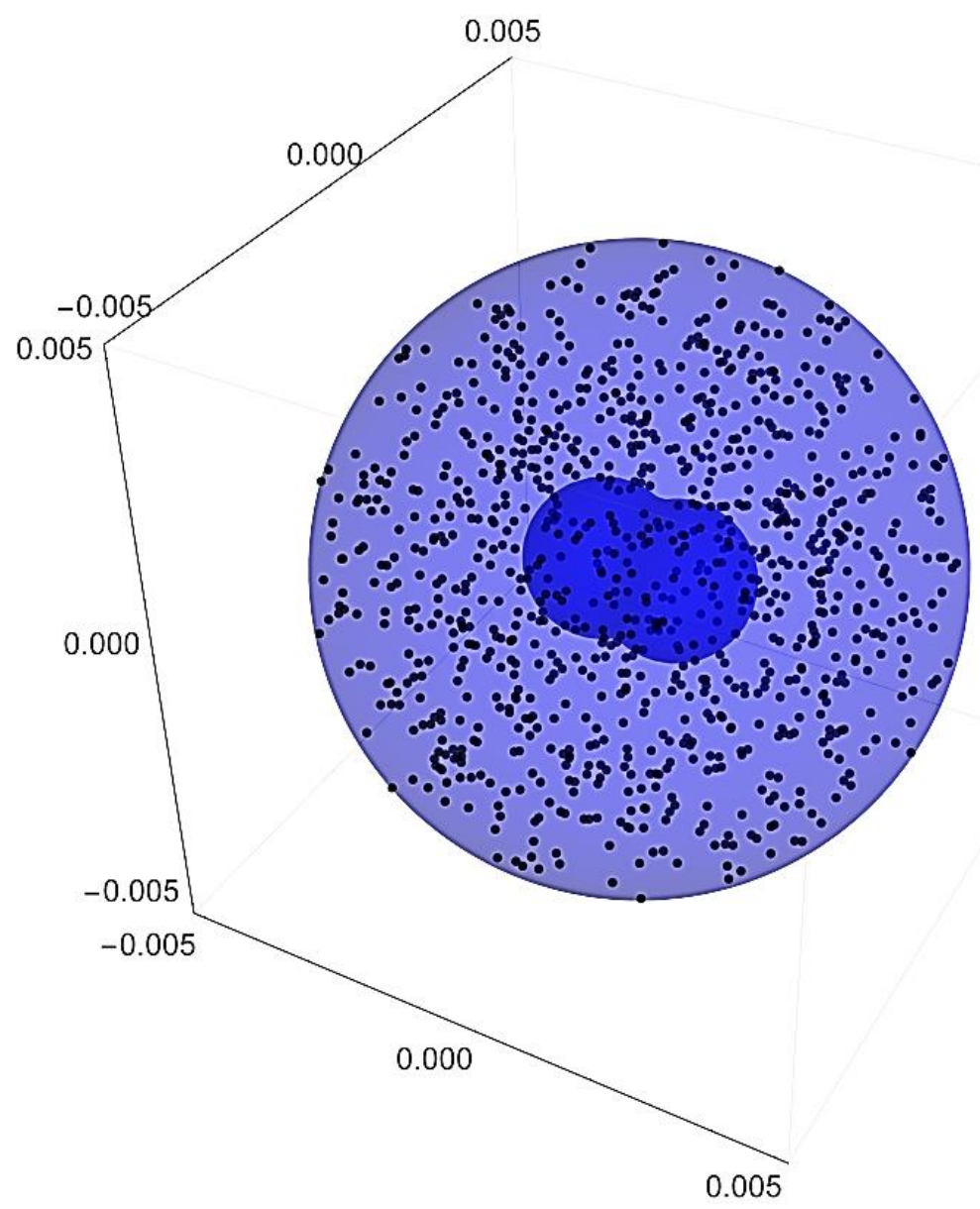

Figure 13: Spherical Vessel with Internal Obstruction and Aerosol Particles 


\section{RESULTS: DEPOSITON}

Each vessel geometry described in the preceding chapter was subjected to a series of aerosol modeling cases, the results of which are presented in the next few chapters. Two aerosol cases, deposition and deposition with coagulation, were applied to each shape. Additionally, a select few geometries (sphere, cuboid, and sphere with internal obstruction) were used to simulate the aerosol case combining deposition, coagulation, and condensation processes.

For every aerosol simulation, the initial particle distribution took the form of a lognormal distribution,

$$
p(d)=\frac{1}{\sqrt{2 \pi \sigma}} \frac{1}{d} \operatorname{Exp}\left[-\frac{1}{2 \sigma}(\log [d / \bar{d}])^{2}\right] \quad \text { Eq. } 38
$$

where $\bar{d}=0.1 \mu \mathrm{m}$ and $\sigma=0.035$. The resulting plot is shown as the line in Figure 14 . Using a DSMC selection-rejection technique, an initial list of $10^{5}$ representative particles were also created, sized according to the lognormal distribution. These DSMC calculations are represented by the bars in the figure.

To facilitate the computations, several initial parameters had to be defined. These parameters include particle number density, particle mass density, and the total number of components that constitute an aerosol particle. Since they don't depend on the particle processes being employed (e.g. coagulation) and are also spatially independent, these parameter values were used in all of the DSMC calculations. A list of these parameters is provided in Table 2. Each simulation was computed ten distinct times $\left(N_{\text {Runs }}=10\right)$, with the output data being averaged to determine the final results. 


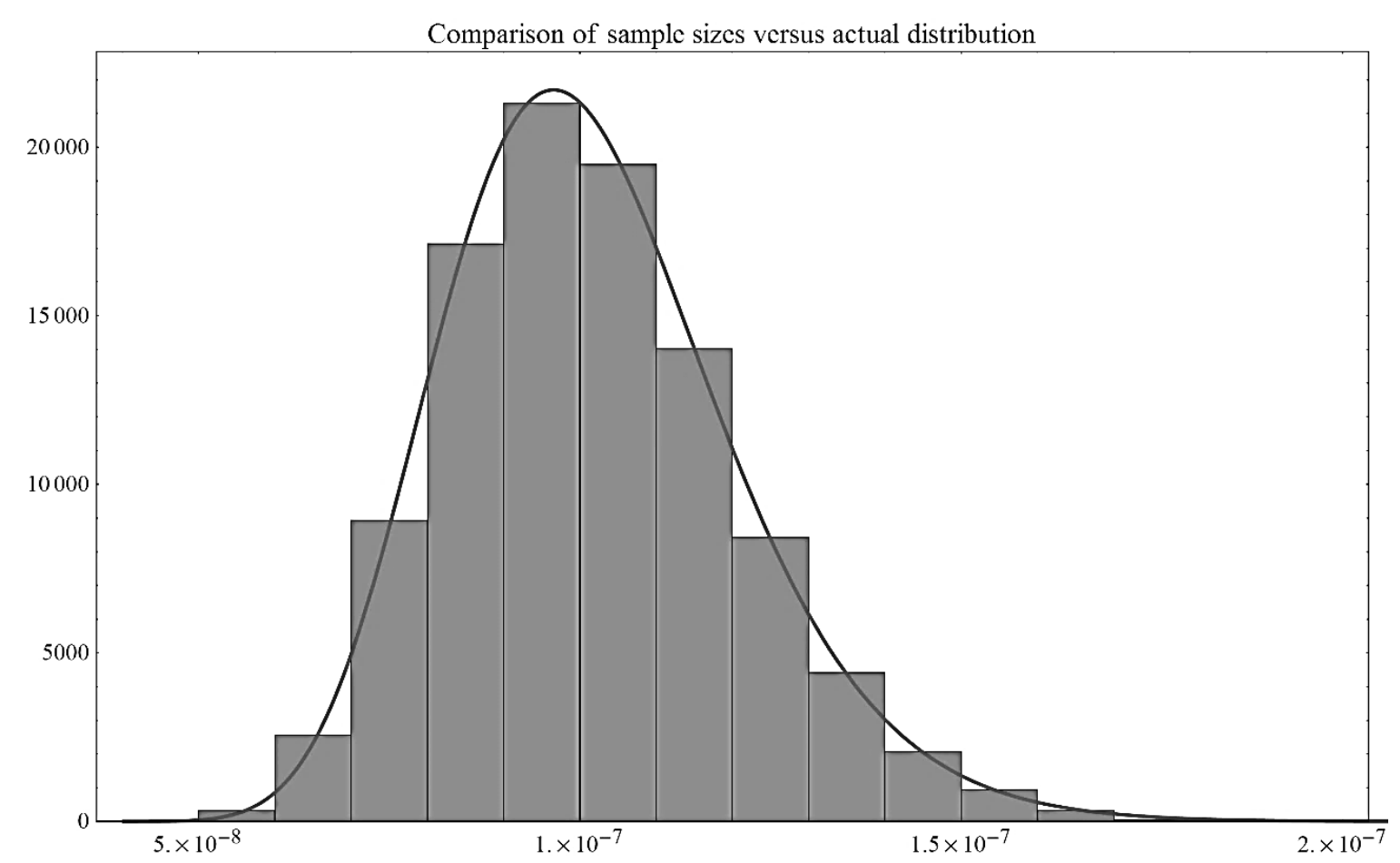

Figure 14: Comparison of Initial Aerosol Particle Size Distribution

Table 2: Initial Parameters for All DSMC Simulations

\begin{tabular}{|l|l|}
\hline Initial Parameters & $N_{P}=10^{14}$ particles $/ \mathrm{m}^{3}$ \\
\hline Particle number density & $\rho=1000 \mathrm{~kg} / \mathrm{m}^{3}$ \\
\hline Particle mass density & $k_{\text {Total }}=1$ \\
\hline Number of particle components & $N=10^{5}$ \\
\hline Time step duration & $\Delta t=0.1$ seconds \\
\hline Brownian time step duration & $\Delta t_{\text {Brown }}=0.01$ seconds \\
\hline DSMC runs per simulation & $N_{\text {Runs }}=10$ \\
\hline
\end{tabular}

In addition to defining parameter values used in all of the DSMC calculations, a few simplifying assumptions were made. As discussed previously, it was assumed that particle diffusion behavior could be fully attributed to Brownian motion. This limits the complexity of the diffusion phenomenon. Another assumption made for the program was that any particles that come into contact with a boundary deposit on the surface of the boundary. Making this assumption eliminates the need to consider rebound effects, which is potentially an area for future investigation. 
After having determined the parameters and assumptions that applied to all three aerosol modeling cases, the case-specific values also had to be selected. Chosen values for these variables are given in Table 3. The first case-specific values that were assigned were the True/False binary switches. As was discussed in the chapter on program development and modifications (Chapter V), these statements allow the user to enable and disable desired aerosol behaviors. Another case-specific parameter that needed to be decided was the program evaluation time(s) $t$. These $t$ values are used in the second computational code to designate the simulation time(s) at which the program is supposed to create output information such as tables and graphs. The end time $t_{E n d}$ denotes the simulation time at which the aerosol model is to stop conducting computations. Finally, the data file time step $\Delta t_{\text {Data }}$ tells the main program how many simulated seconds to wait before a data file is created and automatically saved in a file directory.

Table 3: Case-Specific Parameters

\begin{tabular}{|l|l|l|l|}
\hline & Deposition Only & $\begin{array}{c}\text { Deposition and } \\
\text { Coagulation }\end{array}$ & $\begin{array}{c}\text { Deposition, } \\
\text { Coagulation, and } \\
\text { Condensation }\end{array}$ \\
\hline $\begin{array}{l}\text { Process Kernels } \\
\text { (Binary Switches) }\end{array}$ & $\begin{array}{l}\text { depKernel }=\text { True } \\
\text { coagKernel }=\text { False } \\
\text { condKernel }=\text { False }\end{array}$ & $\begin{array}{l}\text { depKernel }=\text { True } \\
\text { coagKernel }=\text { True } \\
\text { condKernel }=\text { False }\end{array}$ & $\begin{array}{l}\text { depKernel }=\text { True } \\
\text { coagKernel }=\text { True } \\
\text { condKernel }=\text { True }\end{array}$ \\
\hline Evaluation Times & $t=50,100$ seconds & $t=10,20$ seconds & $t=10,20$ seconds \\
\hline End Time & $t_{\text {End }}=100$ seconds & $t_{\text {End }}=20$ seconds & $t_{\text {End }}=20$ seconds \\
\hline $\begin{array}{l}\text { Data File } \\
\text { Time Step }\end{array}$ & $\Delta t_{\text {Data }}=5$ seconds & $\Delta t_{\text {Data }}=1$ second & $\Delta t_{\text {Data }}=1$ second \\
\hline
\end{tabular}

For the "Deposition Only" aerosol model analyzed in this chapter, the text-based switches for both coagulation and condensation were turned to "False" in Table 3. This means that all the results are solely due to the effects of the deposition process in each geometry. Additionally, graphical results were generated for each geometry vessel at 50 
seconds (halfway through the simulation) and 100 seconds (at the end of the simulation).

Data files were written to the directory in 5 second intervals. Results for each individual geometry are given below.

\section{Sphere}

Having decided the parameter values for each aerosol model case, the "Deposition Only" program settings were applied to a spherical vessel. This geometry was done first in order to establish a benchmark with prior literature. In order to compare the particle suspension behavior with the results from Campbell et al. ${ }^{33}$, the sphere had to be divided into "shells." These shells are concentric and correspond to the volumetric change $d V$ (see Figure 15).To determine the spatial distribution of the aerosol at a given time, the masses of all particles within the shell boundaries were summed. For the spherical vessel aerosol simulations, the total number of shells $I$ was assigned the value of 100 .

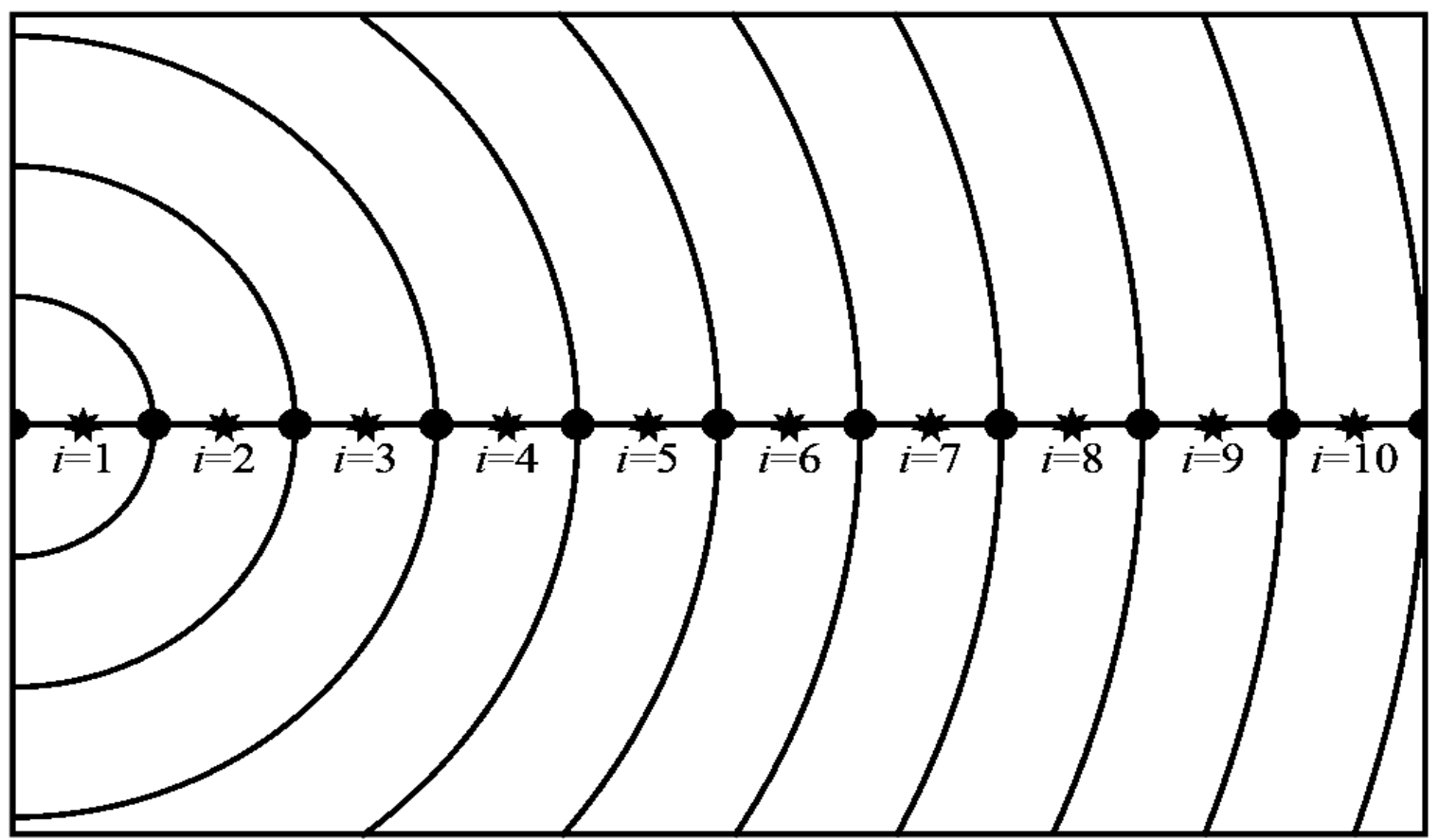

Figure 15: A Sphere of Radius $R$, Split Into Ten Shells 
The next two figures (Figure 16 and Figure 17) correspond to the particle suspension behavior within the spherical geometry at 50 seconds and 100 seconds, respectively. Comparing these results to those provided in the study by Campbell et al. ${ }^{33}$, it can be seen that the outputs are exceedingly similar. For the first evaluation time, both plots peak at slightly over $0.70 \mathrm{ng}$ at cell 90 before dropping off sharply as the cell number continues to rise. At the second evaluation time the peaks are less, topping out at approximately $0.65 \mathrm{ng}$.

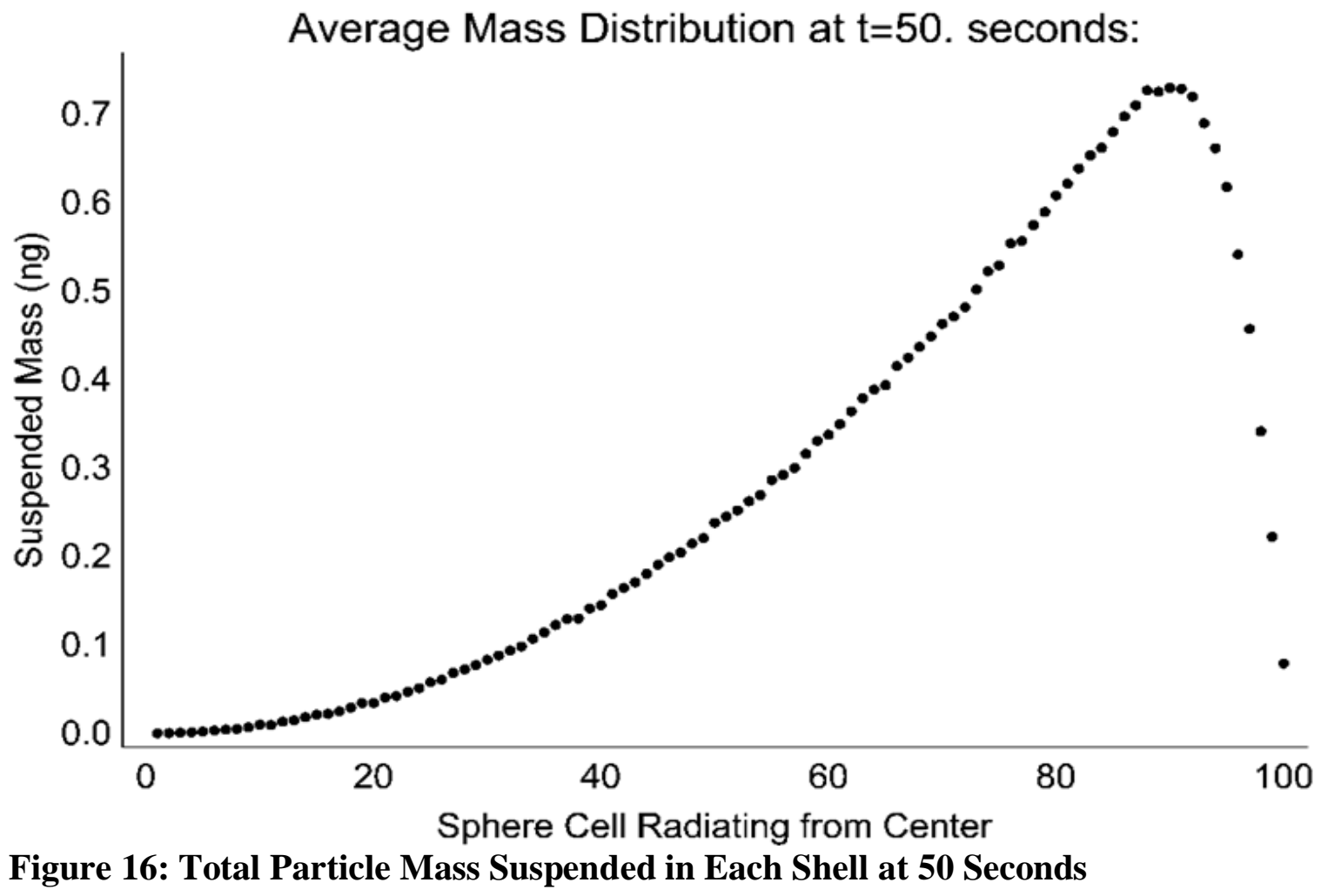

Another way to represent the data given in these two figures is in the form of mass concentration. For the sphere, the mass concentration could be calculated in two different ways. The first approach took advantage of the sphere's spherical symmetry by simply dividing the mass in each cell by the cell's volume. Unfortunately, this method does not work on non-symmetric geometries. To overcome this problem, a second 
approach was developed using a series of 45 sample points spaced along an arbitrary axis. Around each point a small "sample sphere" was created, and the local concentration within that sphere was determined.

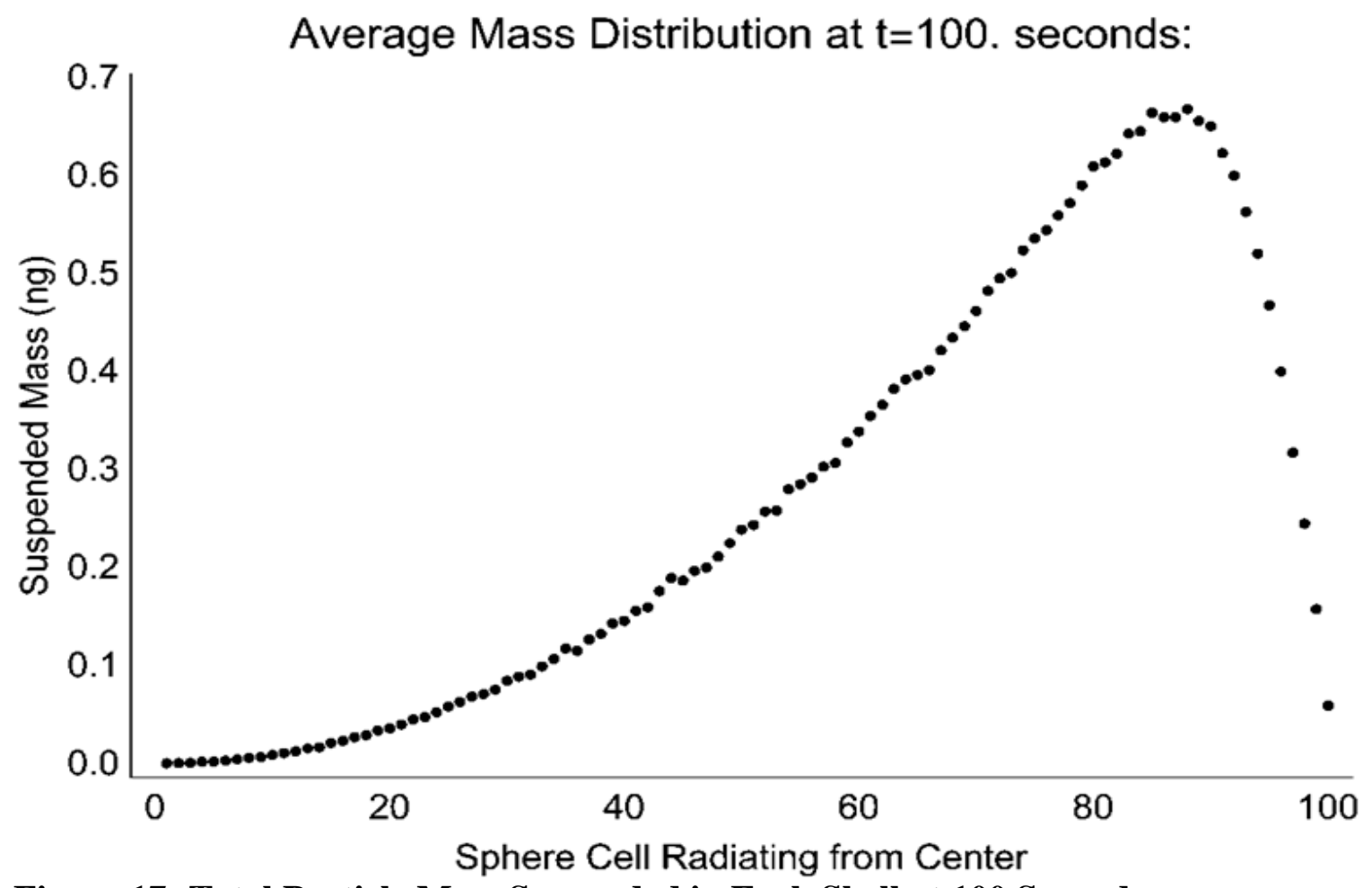

Figure 17: Total Particle Mass Suspended in Each Shell at 100 Seconds

In order to ensure that the two mass concentration approaches produced the same results, both techniques were compared at 50 seconds and 100 seconds. The first two figures (Figure 18 and Figure 19) show the average mass concentration 50 seconds into the aerosol simulation, as calculated by both procedures. Similarly, the average mass concentration at the end of the aerosol simulation is given in Figure 20 and Figure 21 for both approaches. Comparing these graphs indicate that the "Sample Sphere" technique does a decent job of determining mass concentration, even when constrained by the random nature of DSMC. 


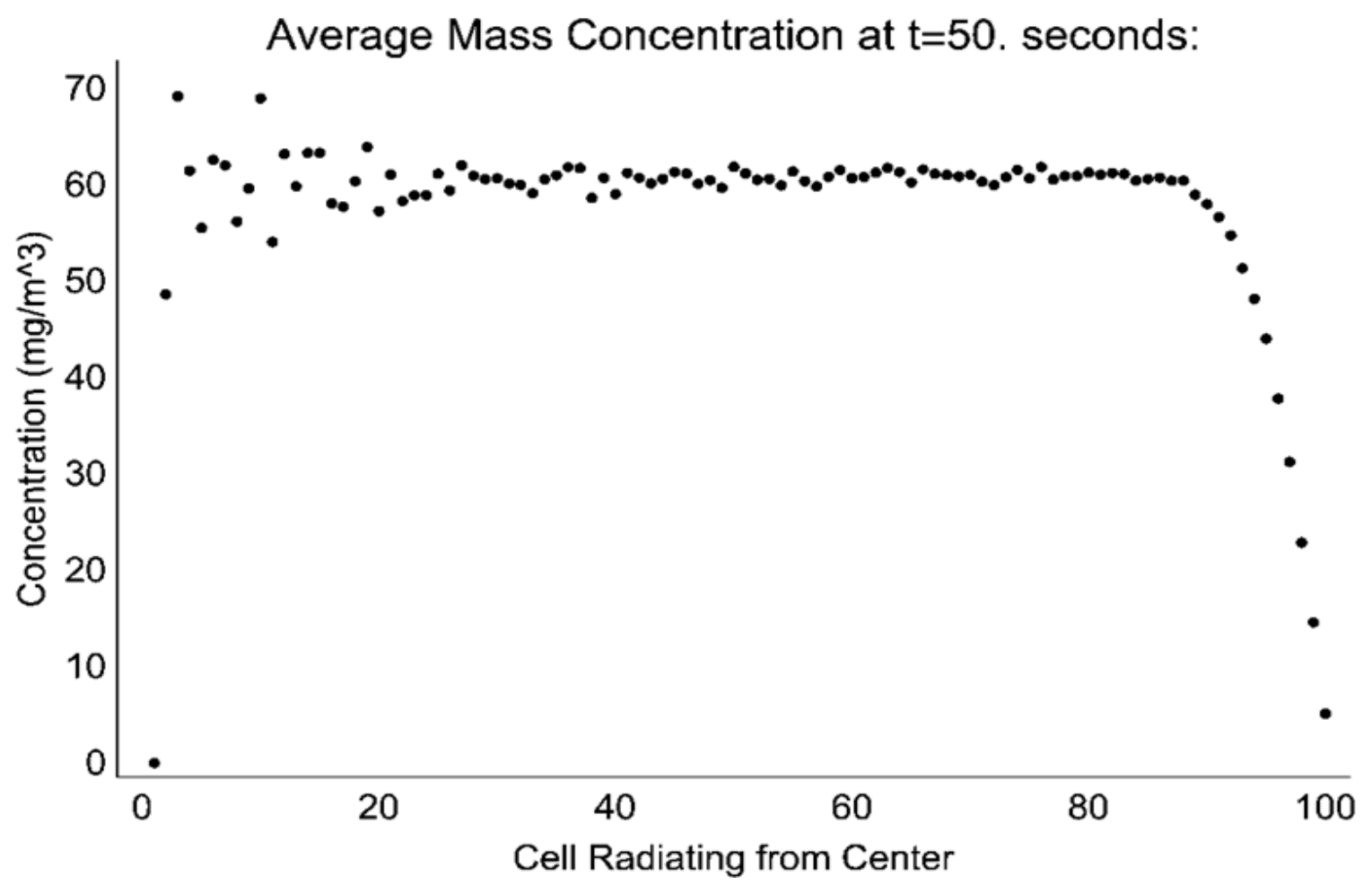

Figure 18: Mass Concentration at 50 Seconds, Using Spherical Symmetry

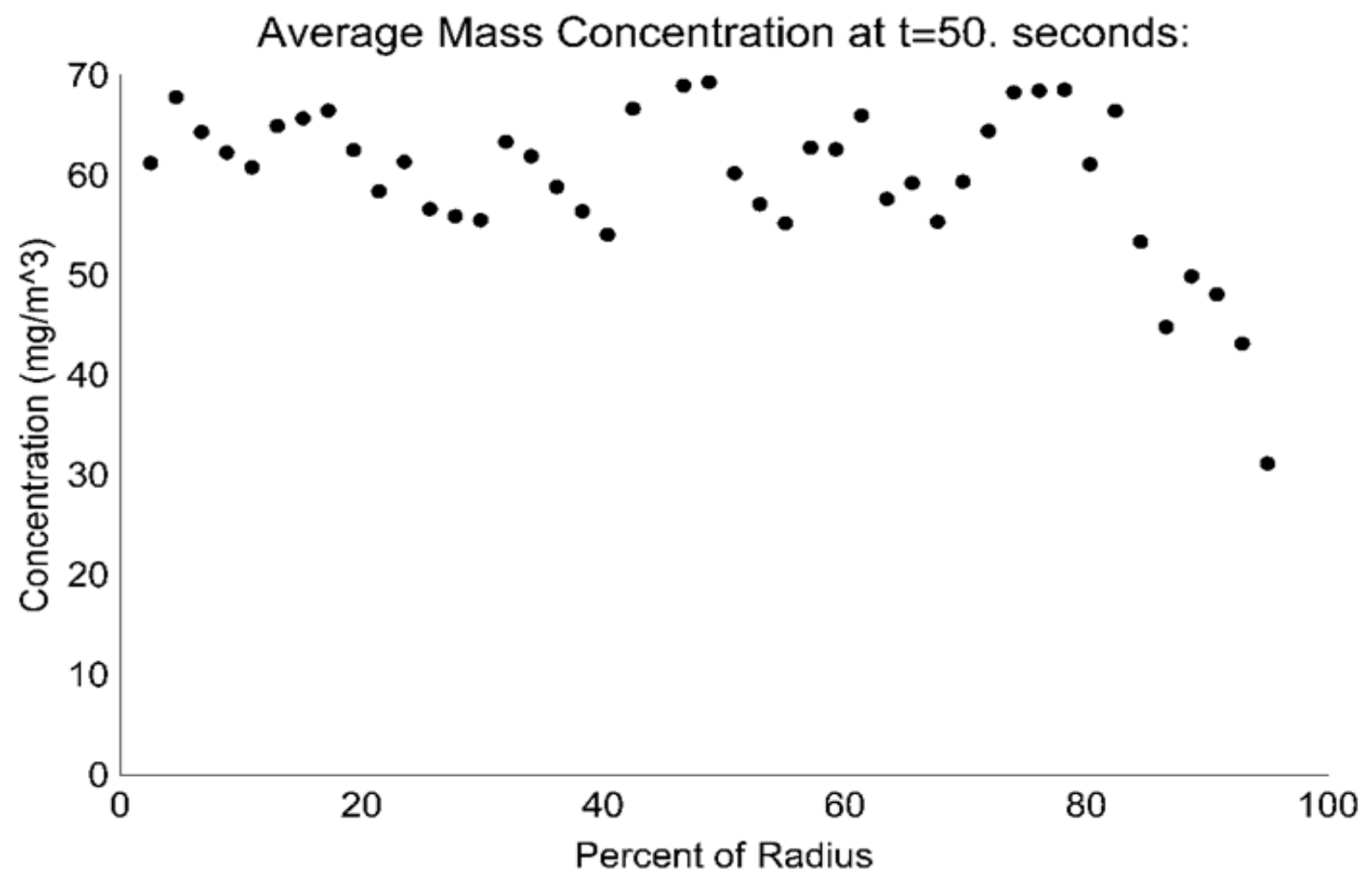

Figure 19: Mass Concentration at 50 Seconds, Using Sample Spheres 


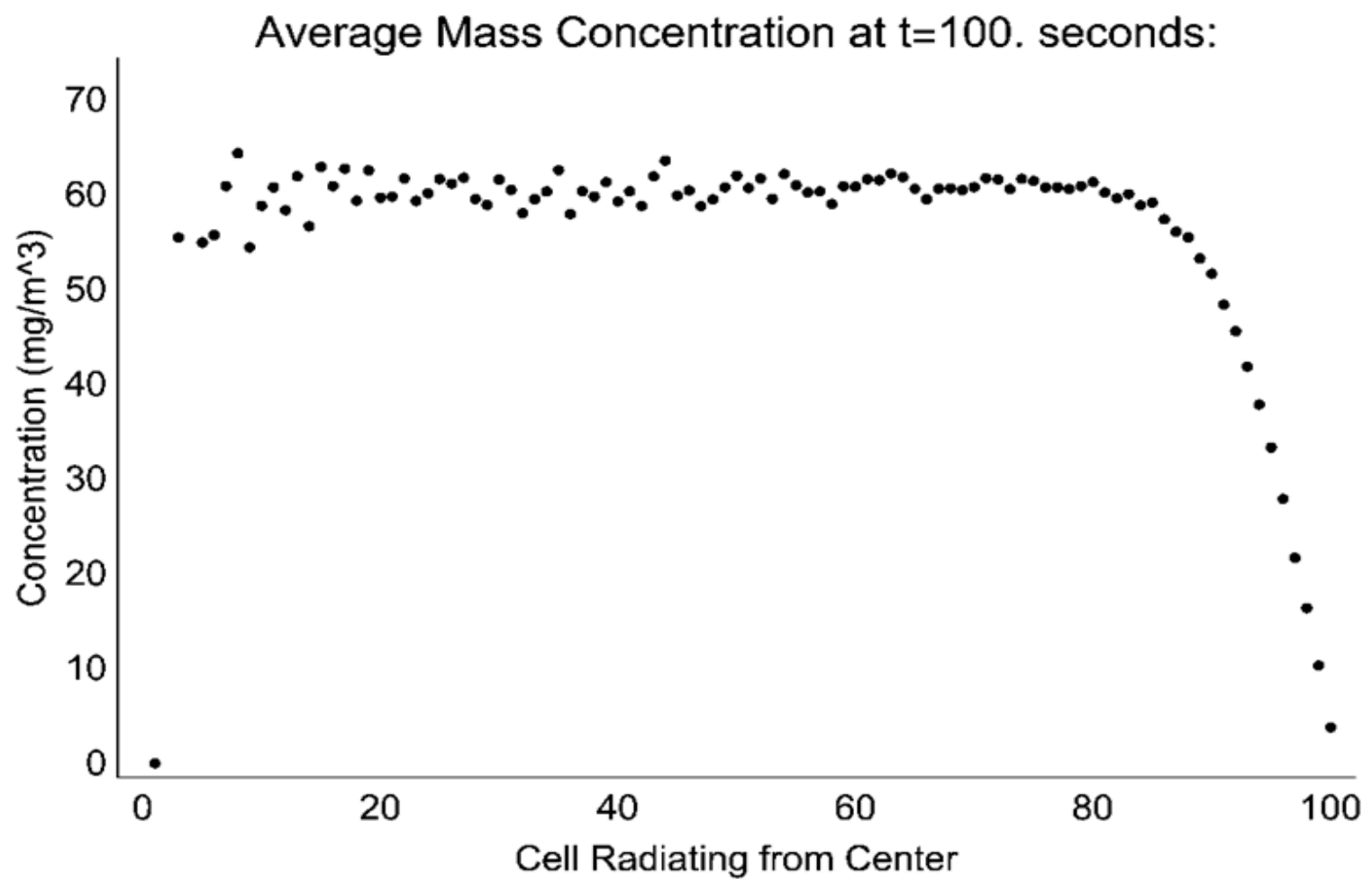

Figure 20: Mass Concentration at 100 Seconds, Using Spherical Symmetry

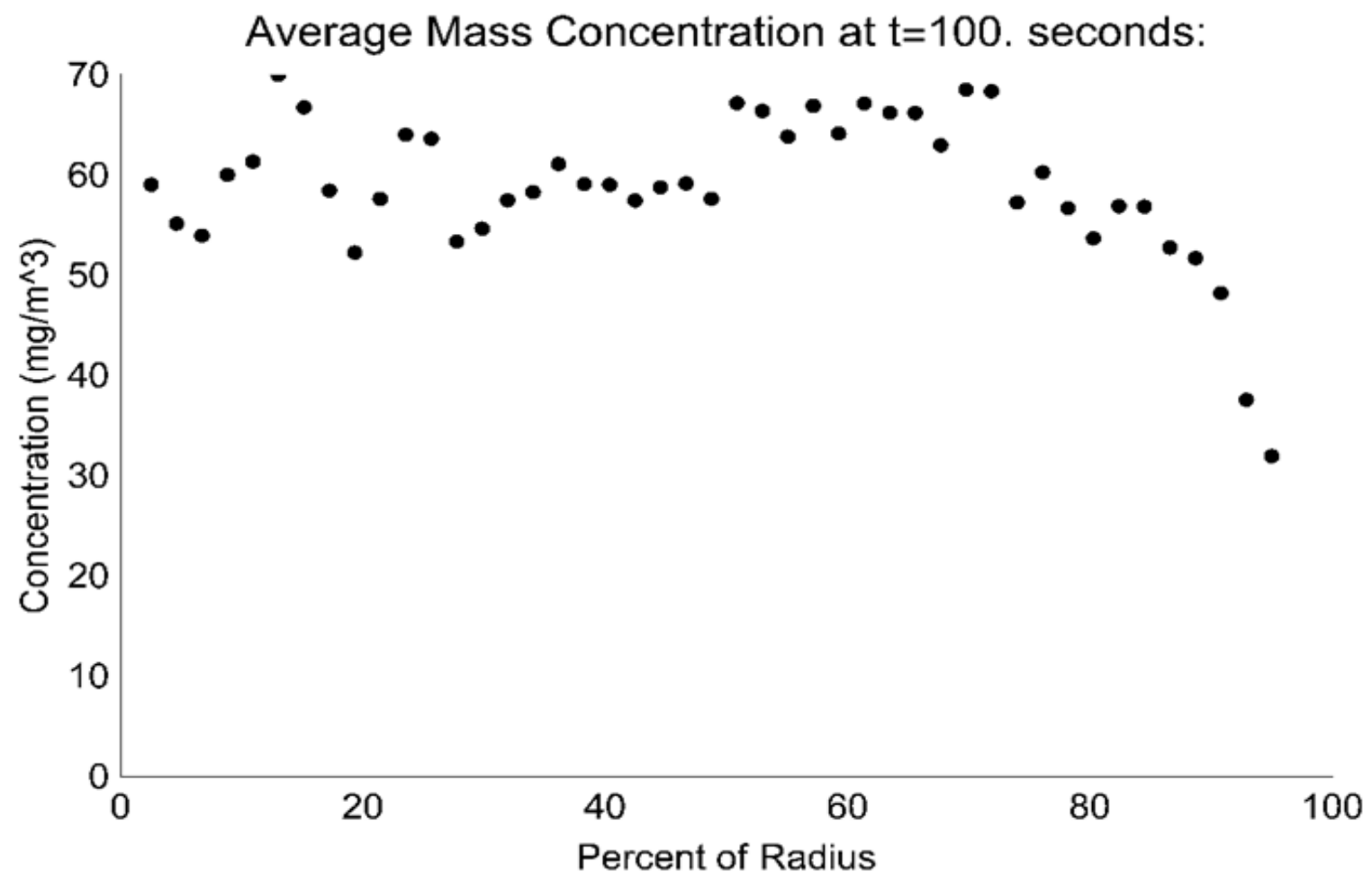

Figure 21: Mass Concentration at 100 Seconds, Using Sample Spheres 
By analyzing the four figures above, it is readily apparent that for $t=50$ seconds and $t=100$ seconds, both methods yield a concentration of approximately $60 \mathrm{mg} / \mathrm{m}^{3}$. However, as the interior boundary is approached, the concentration declines. This is due to the deposition of particles on the interior boundary of the vessel. It is important to note that the slight variations in the concentration values given in Figure 19 and Figure 21 are due to randomness of the DSMC approach in regards to particle location.

Cumulative particle deposition was also determined for this simulation. Figure 22 shows this deposition for the 100 second "Deposition Only" case inside a spherical vessel. For this graph, the line denotes the DSMC results while the discrete points correspond to the Finite Element Method (FEM). As can be seen, the cumulative deposition climbs quickly for the first five seconds before slowly starting to taper off. At the end of the simulation, approximately $5 \mathrm{ng}$ of aerosol particles have deposited. Comparing this result to the one given in the study by Campbell et al. ${ }^{33}$ verifies the accuracy of the spatially inhomogeneous aerosol model.

To further verify the accuracy of the spherical vessel, the DSMC results were also compared to FEM results. The close correspondence between the DSMC and FEM values can be seen in Figure 22, and indicate that DSMC can be used as a close approximation of the FEM technique. This is advantageous due to the weaker relationship the mesh-free DSMC approach has with the vessel geometry, resulting in a less computationally demanding simulation. The heavy computational demands of FEM are apparent in the small number of discrete points provided in Figure 22. These were limited by the computational capabilities of the computer as well as the computational time required to conduct the calculations. 


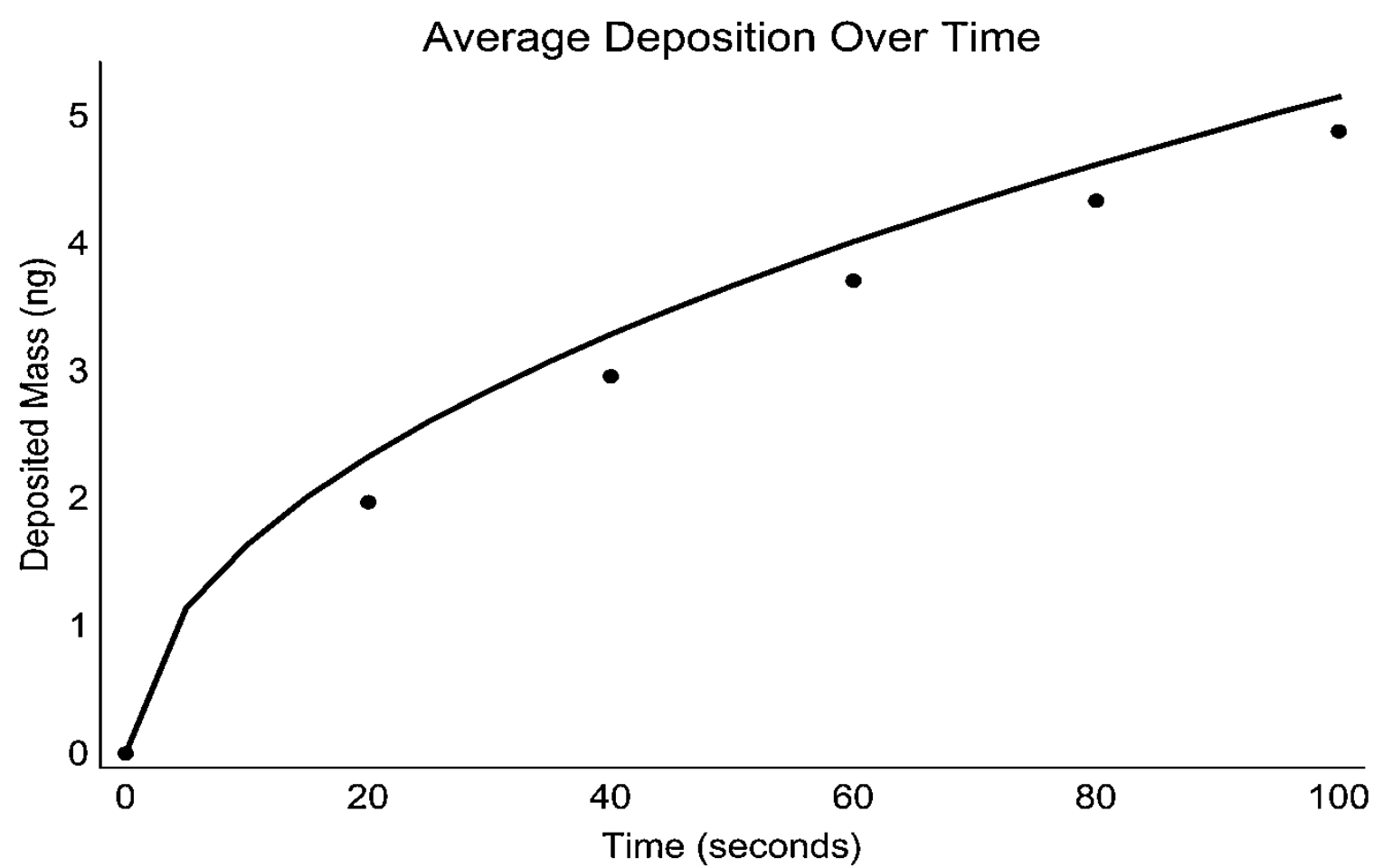

Figure 22: Cumulative Particle Deposition on Inner Surface Of Sphere, for DSMC (Line) and FEM (Points)

\section{Ellipsoid}

Due to the equational similarity between a sphere and an ellipsoid, the ellipsoid was selected as the first new geometry to be evaluated in the program. The results for the "Deposition Only" case for $t=50$ seconds and $t=100$ seconds are given in Figure 23 and Figure 24. Due to the vessel shape, only the "Sample Sphere" technique was used. This technique was employed along the positive $x$ axis. Just like with the spherical vessel, the mass concentration maintains a value of approximately $60 \mathrm{mg} / \mathrm{m}^{3}$, and declines as the interior boundary is approached. This suggests that $60 \mathrm{mg} / \mathrm{m}^{3}$ is a saturation value directly related to the particle number density $N_{P}=10^{14}$ particles $/ \mathrm{m}^{3}$ given in Table 2 .

The concentration decline when the interior boundary is approached is again attributed to particle deposition effects. Slight fluctuations in the concentration values presented in the two figures are due to randomness of particle location in the DSMC approach. 


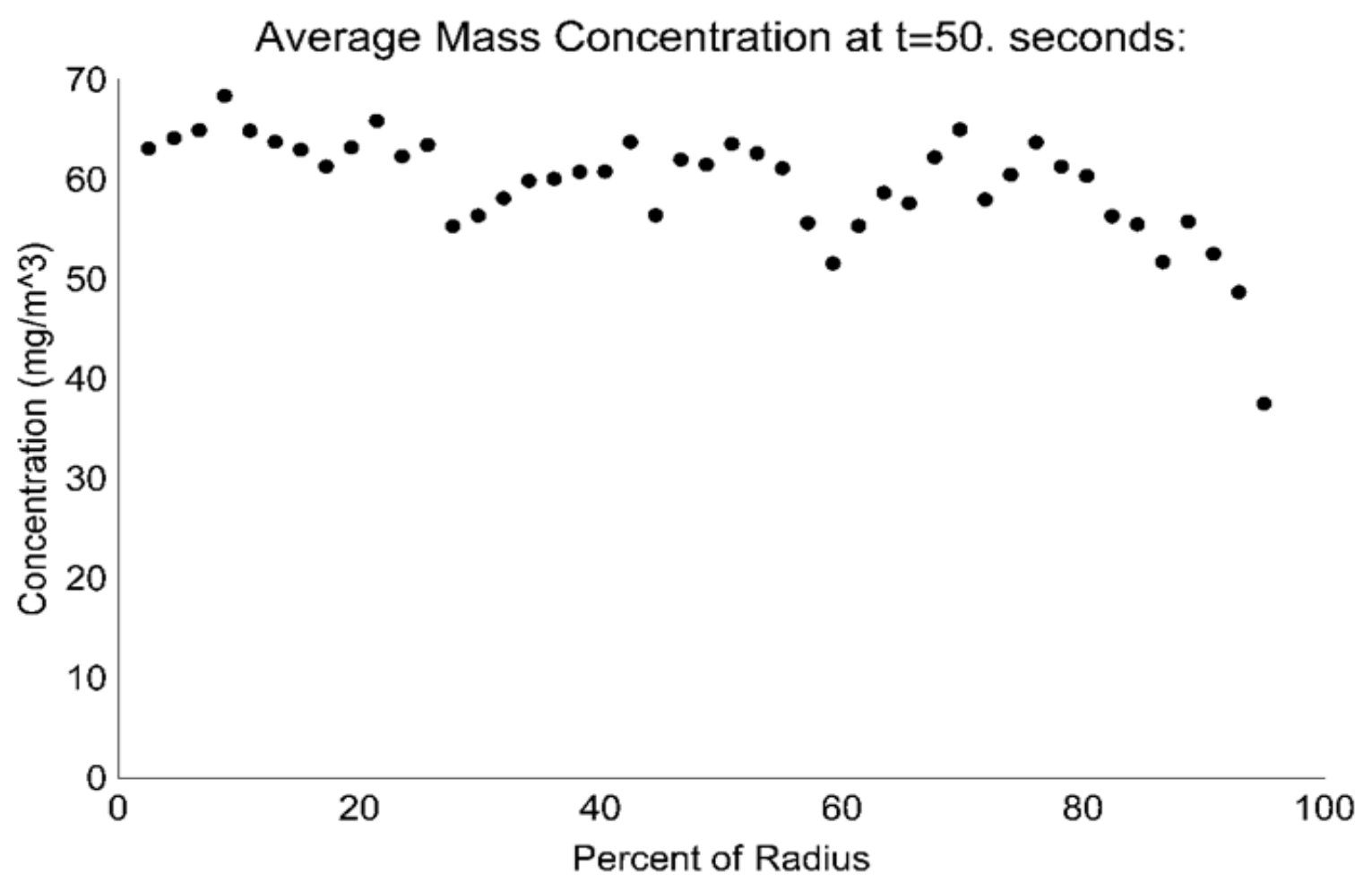

Figure 23: Mass Concentration of Ellipsoid at 50 Seconds

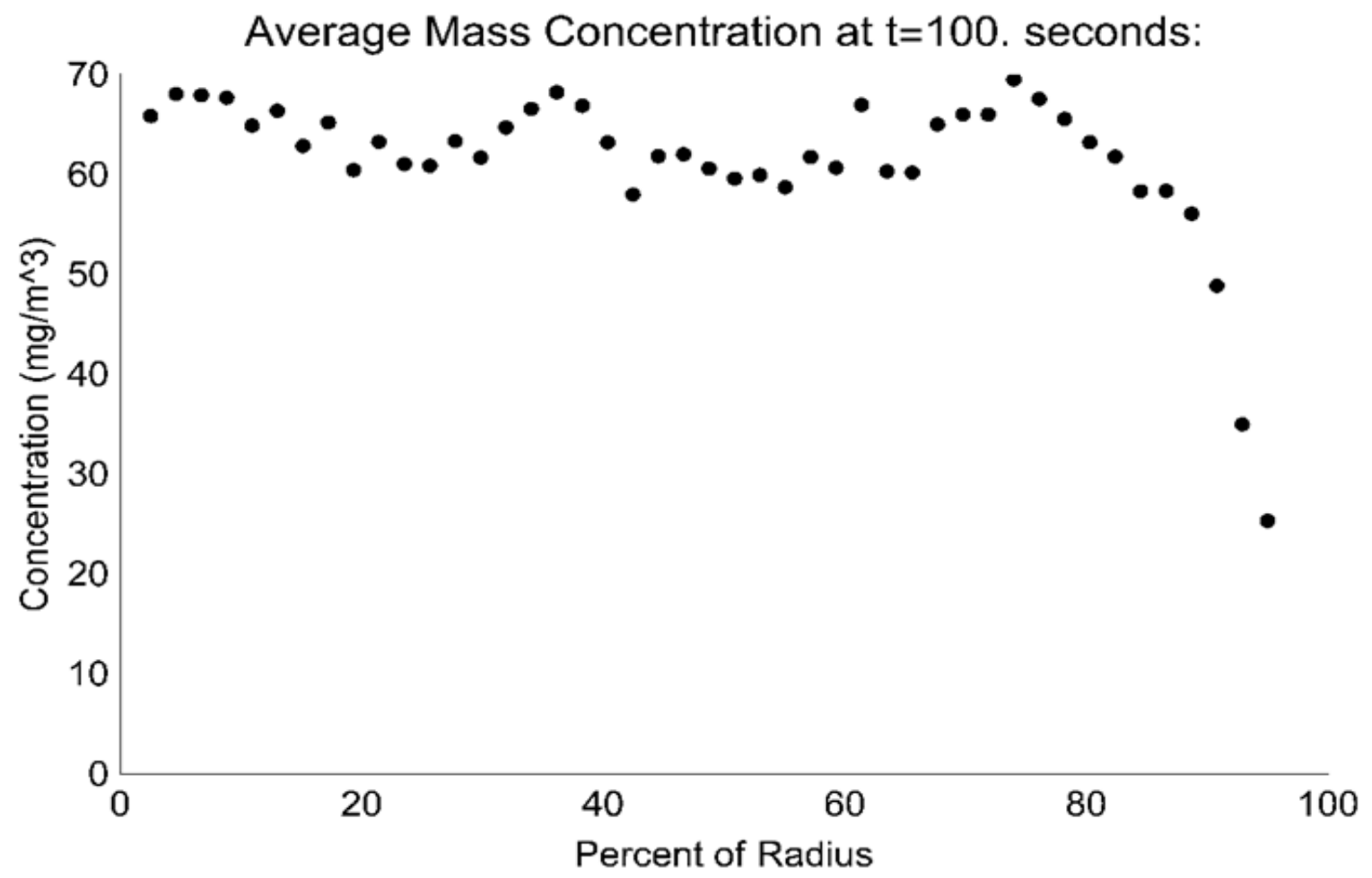

Figure 24: Mass Concentration of Ellipsoid at 100 Seconds 


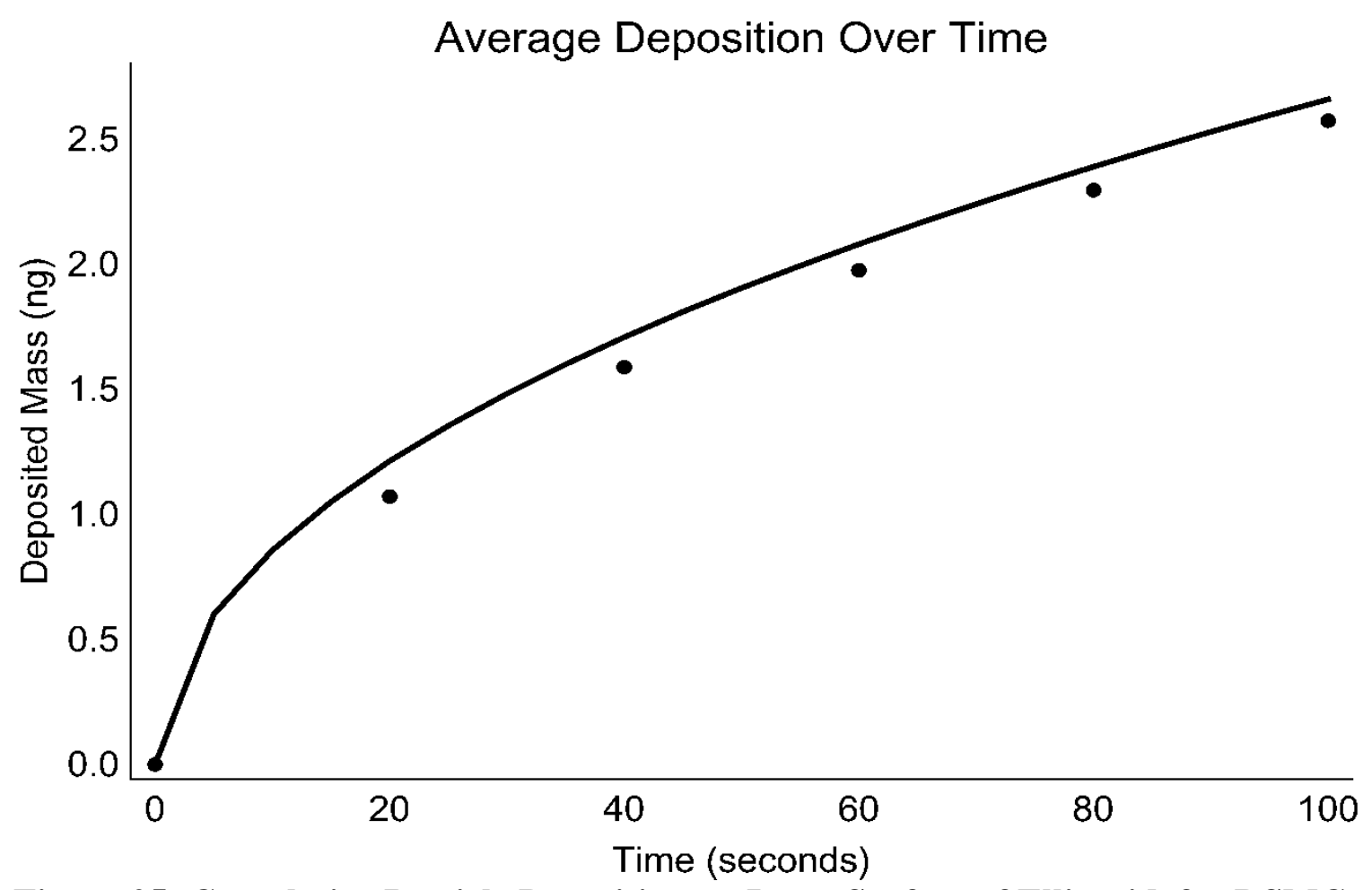

Figure 25: Cumulative Particle Deposition on Inner Surface of Ellipsoid, for DSMC (Line) and FEM (Points)

In addition to the local mass concentration, the cumulative particle deposition was also calculated. Deposition for the 100 second "Deposition Only" case inside the elliptical vessel can be seen in Figure 25. For this graph, the line denotes the DSMC results while the discrete points correspond to FEM. The cumulative deposition climbs quickly for the first five seconds before slowly starting to taper off, in exactly the same form as for the spherical geometry. At the end of the simulation, approximately $2.5 \mathrm{ng}$ of aerosol particles have deposited. This decrease is credited to the smaller dimensions of the vessel.

In order to verify the accuracy of the DSMC results for the elliptical vessel, the FEM results were also calculated. The close correspondence between the DSMC and FEM values can be seen in Figure 25. This correspondence indicates that DSMC can be used to generate results that are a close approximation to the FEM technique. For this 
geometry, the heavy computational demands of FEM once again restricted the number of points given in the figure. As mentioned previously, these restrictions were due to the computational capacity of the computer as well as the computational time required to run the FEM simulations.

\section{Torus}

After completing the "Deposition Only" aerosol model for the ellipsoid, the calculations were repeated for a torus. Figure 26 and Figure 27 give the results for this case at $t=50$ and $t=100$ seconds, respectively. Since the torus was symmetric about the $z$ axis, the "Sample Sphere" method was used for an arbitrary direction in the $x y$ plane. These spheres extended from the axis origin to the outer boundary of the toroid vessel, corresponding to a total distance of $c+a=4 R$ (see Chapter VI). Note that, for a torus with outer boundary $4 R$ and tube radius $R$, the inner boundary of the tube is at a distance $2 R$ from the $z$ axis. Thus, particles in the graphs should start appearing at $2 R / 4 R=50 \%$ of the total distance. However, both figures indicate that the local mass concentration starts increasing slightly before the $50 \%$ mark.

Fortunately, the reason for this is simply due to the approach used in the "Sample Sphere" technique. With this method, it is possible for a sphere centered just outside a given vessel boundary to have a radius large enough to incorporate some aerosol particles inside the vessel into its calculations. The slight increase in local mass concentration for the torus just before the $50 \%$ mark is due to this effect. After this point, the concentration rises rapidly before leveling off at a value of approximately $60 \mathrm{mg} / \mathrm{m}^{3}$. Slight fluctuations in the concentration values presented in the two figures are due to randomness of particle location in the DSMC approach. 


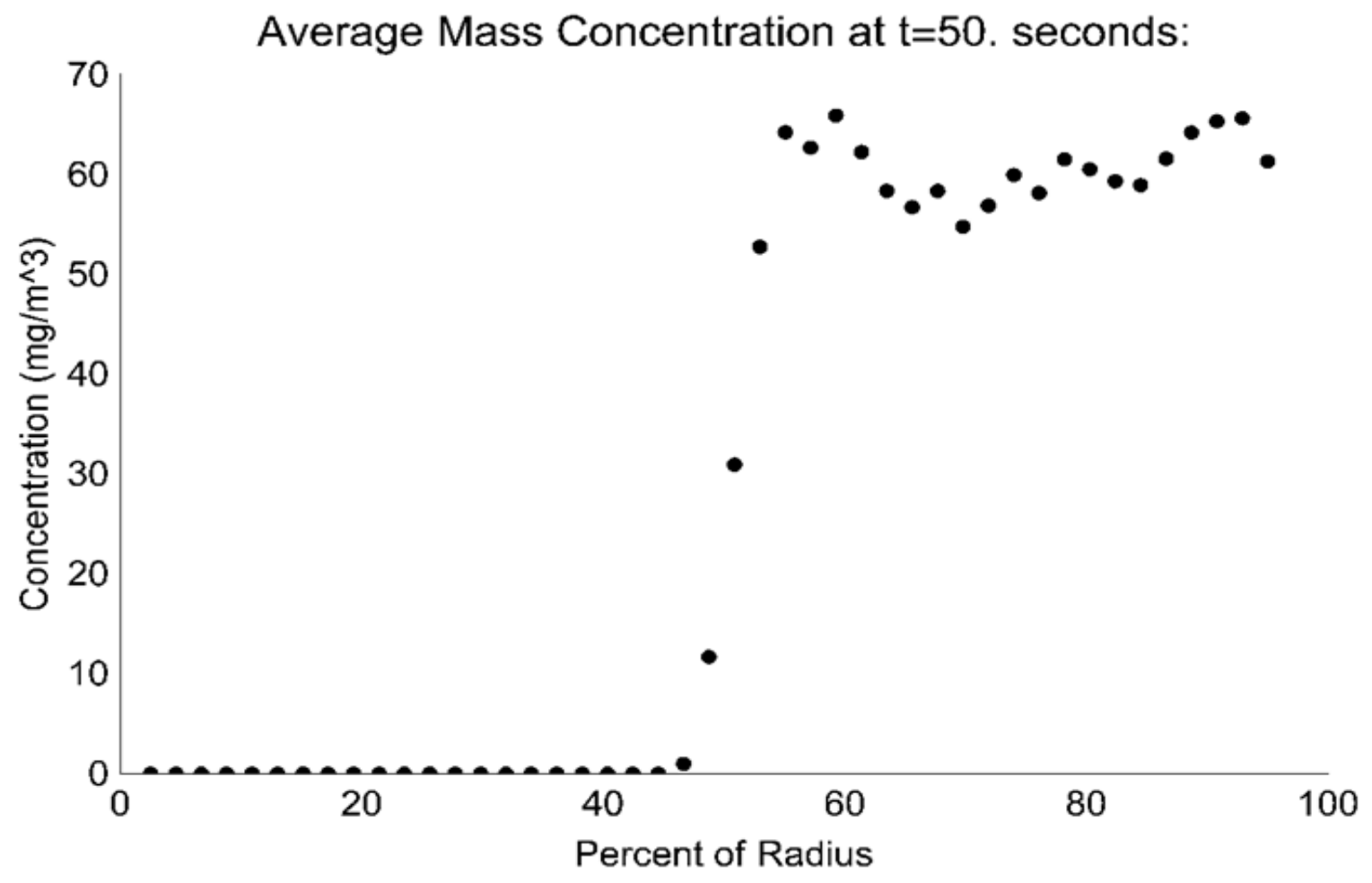

Figure 26: Mass Concentration of Torus at 50 Seconds

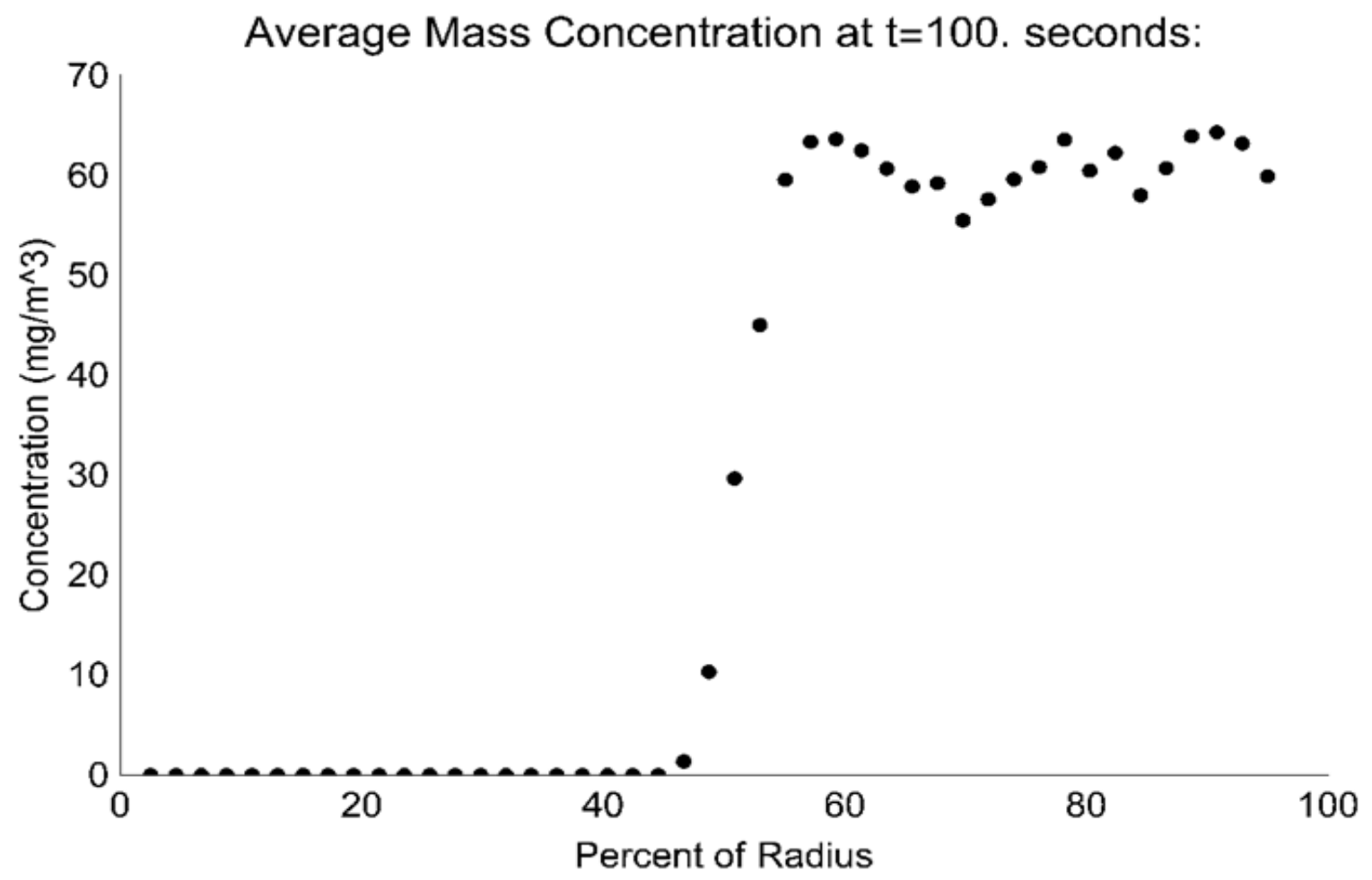

Figure 27: Mass Concentration of Torus at 100 Seconds 
Cumulative particle deposition was also calculated for the torus, and can be seen in Figure 28. Once again, the cumulative deposition climbs sharply for the first few seconds. The deposition rate then slows down in a manner similar to the previous geometries. At the end of the simulation, approximately $50 \mathrm{ng}$ of aerosol particles have deposited. This value is an order of magnitude larger than the previous two vessels and is attributed to the much larger dimensions of the torus.

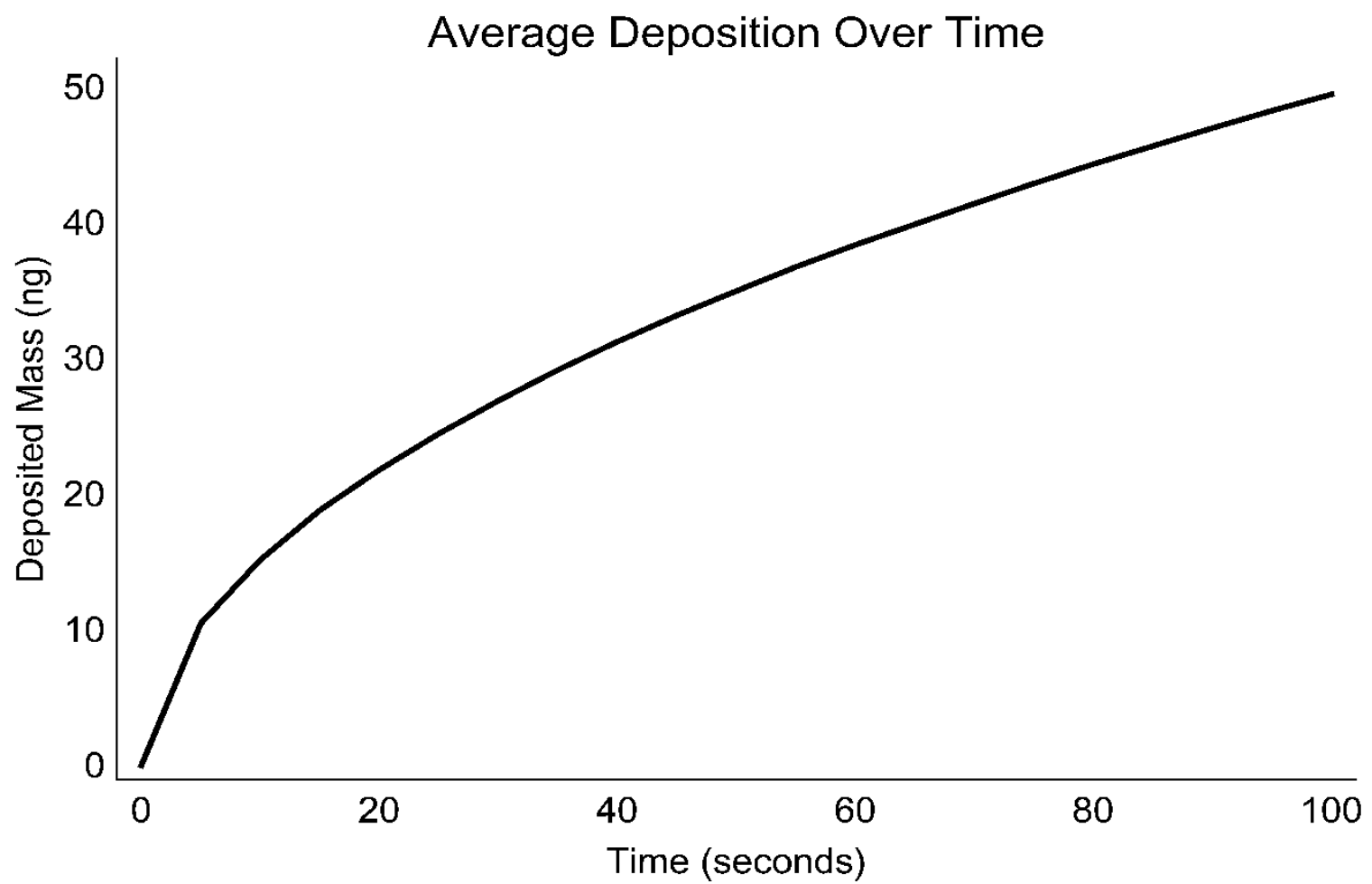

Figure 28: Cumulative Particle Deposition on Inner Surface of Torus

\section{Elliptical Cylinder}

Once simulations for the torus had been completed, evaluations were conducted on the elliptical cylinder. The two physical discontinuities present in this shape provided the opportunity to analyze aerosol particle phenomena in a vessel that was unlike the previous geometries. Results from this analysis are given in Figure 29 and Figure 30 for $t=50$ and $t=100$ seconds. For these figures, the "Sample Sphere" technique was used 
along the positive $z$ axis, corresponding to cylinder height (see Equation 31). Just like with all the previous vessels, the mass concentration maintains a value of approximately $60 \mathrm{mg} / \mathrm{m}^{3}$, and declines as the interior boundary is approached. The concentration decline when the interior boundary is approached is credited to particle deposition effects. Slight fluctuations in the concentration values presented in the two figures are due to randomness of particle location in the DSMC approach.

In addition to the local mass concentration, the cumulative particle deposition was also calculated. Total deposition over time for the cylindrical vessel can be seen in Figure 31. The cumulative deposition behaves exactly the same as for previous vessels, starting with an initial quick climb before tapering off. At the end of the simulation, approximately $7 \mathrm{ng}$ of aerosol particles have deposited. This amount is considered reasonable for the geometry dimensions provided.

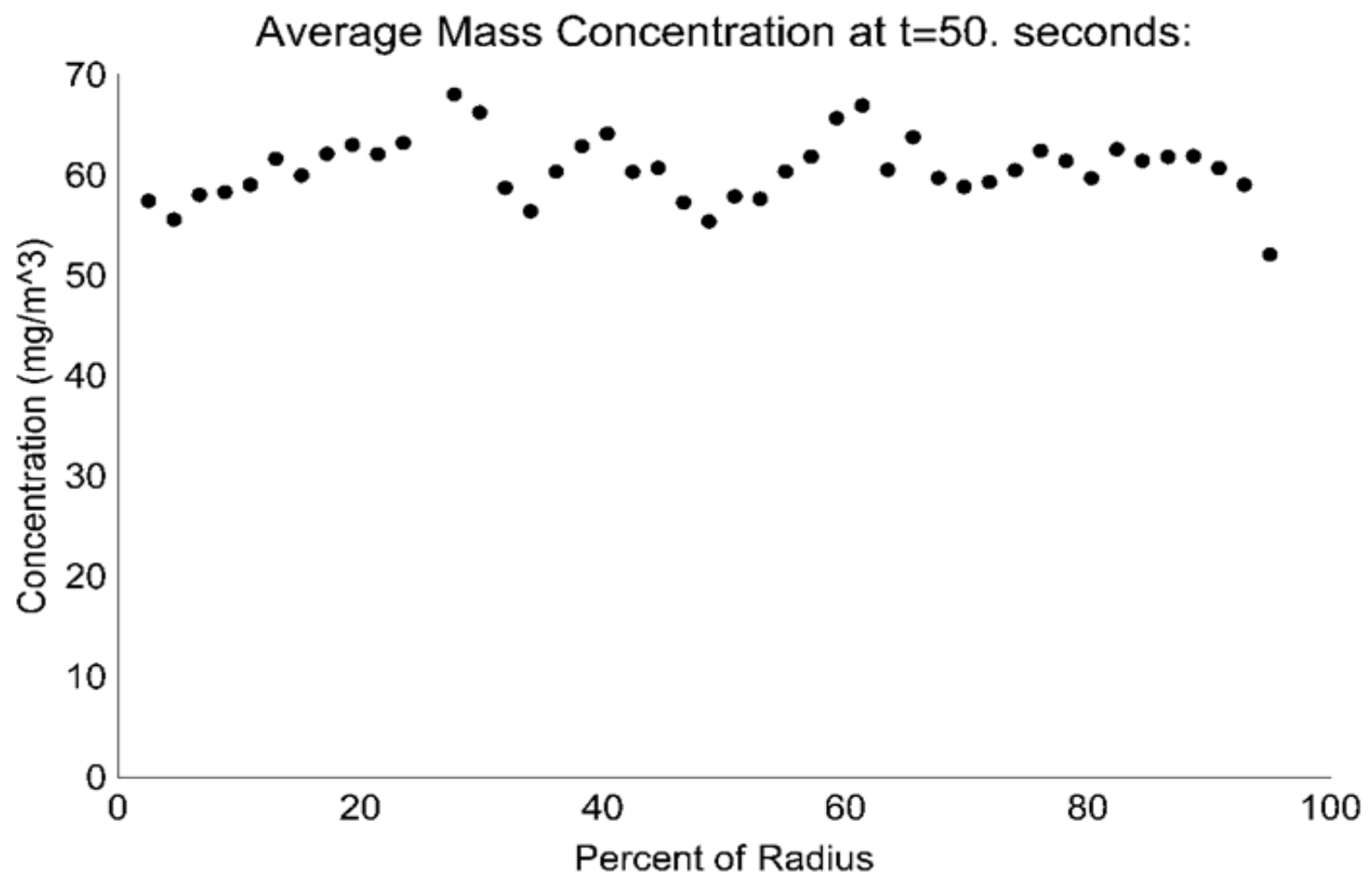

Figure 29: Mass Concentration of Cylinder at 50 Seconds 


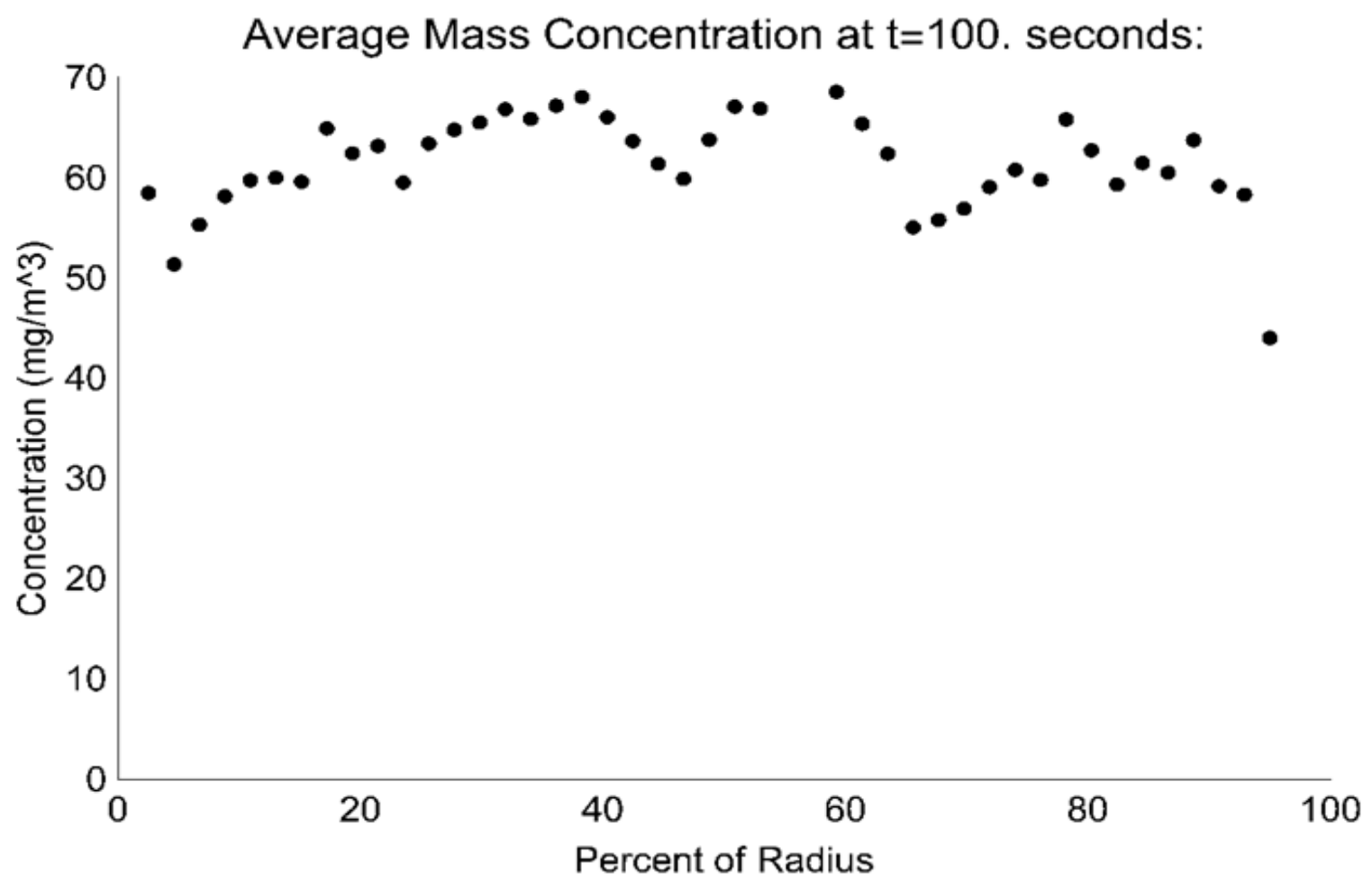

Figure 30: Mass Concentration of Cylinder at 100 Seconds

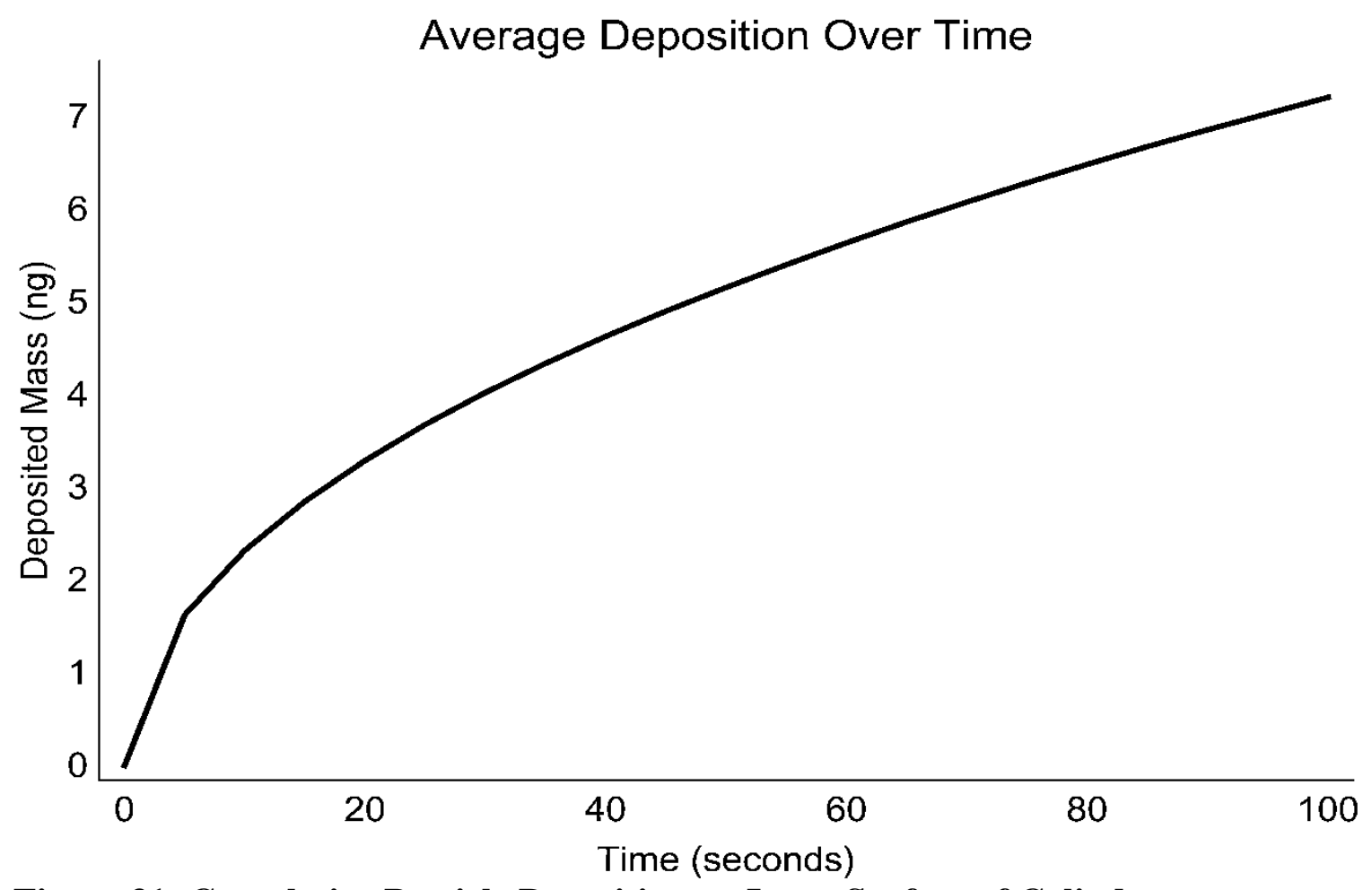

Figure 31: Cumulative Particle Deposition on Inner Surface of Cylinder 


\section{Cuboid}

Since two physical discontinuities were present within the elliptical cylinder, increasing the number of discontinuities was the next logical step in complexity. This resulted in a cuboid geometry being used as the next aerosol vessel. The results for the "Deposition Only" case for $t=50$ seconds and $t=100$ seconds are given in Figure 32 and Figure 33. Due to the vessel shape, only the "Sample Sphere" technique was used. Again, the mass concentration fluctuates around a value of approximately $60 \mathrm{mg} / \mathrm{m}^{3}$, and declines as the interior boundary is approached. Concentration decline when the interior boundary is approached is attributed to the effects of particle deposition. Slight fluctuations in the concentration values for the cuboid are due to randomness of particle location in the DSMC approach.

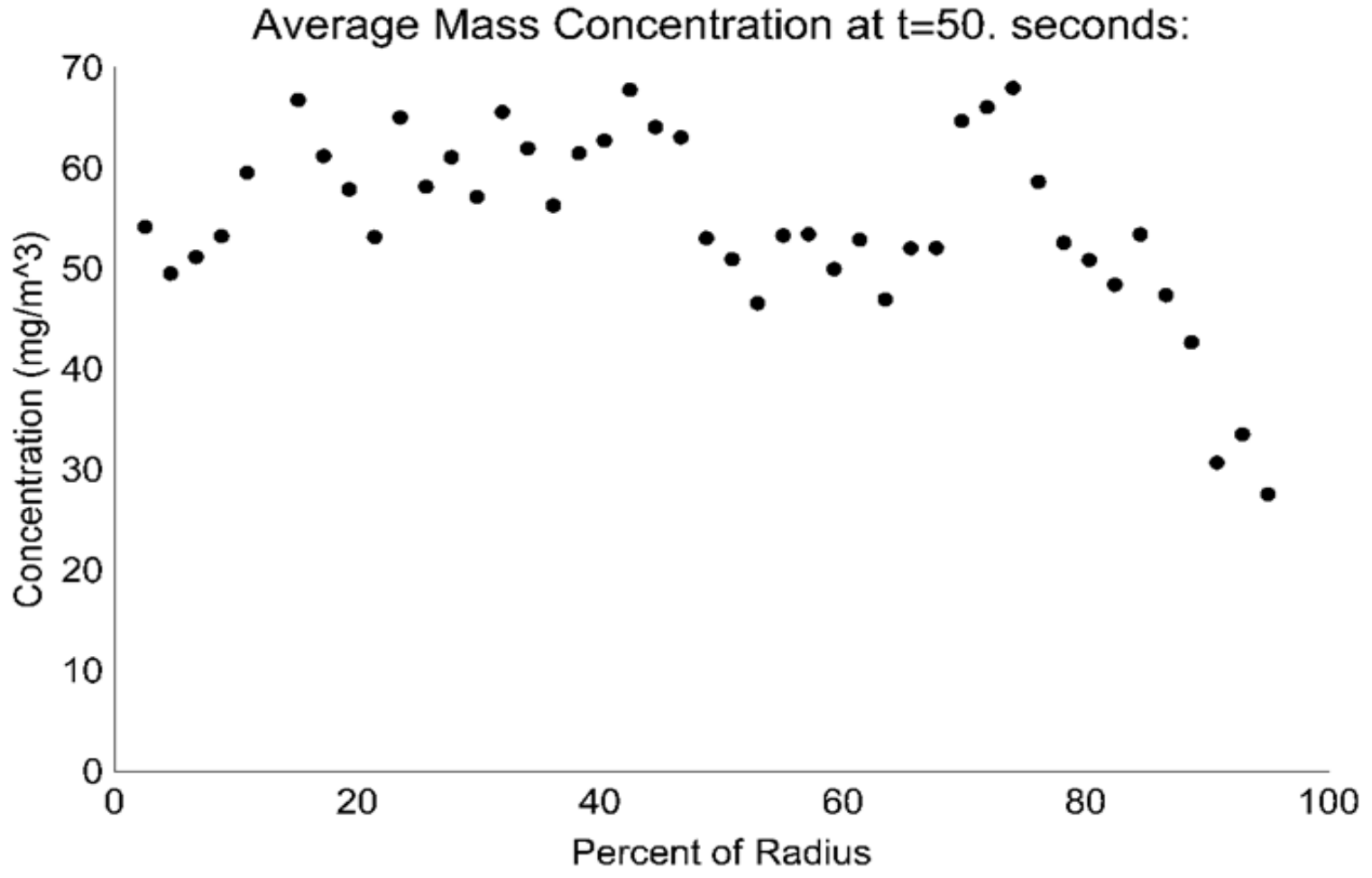

Figure 32: Mass Concentration of Cuboid at 50 Seconds 


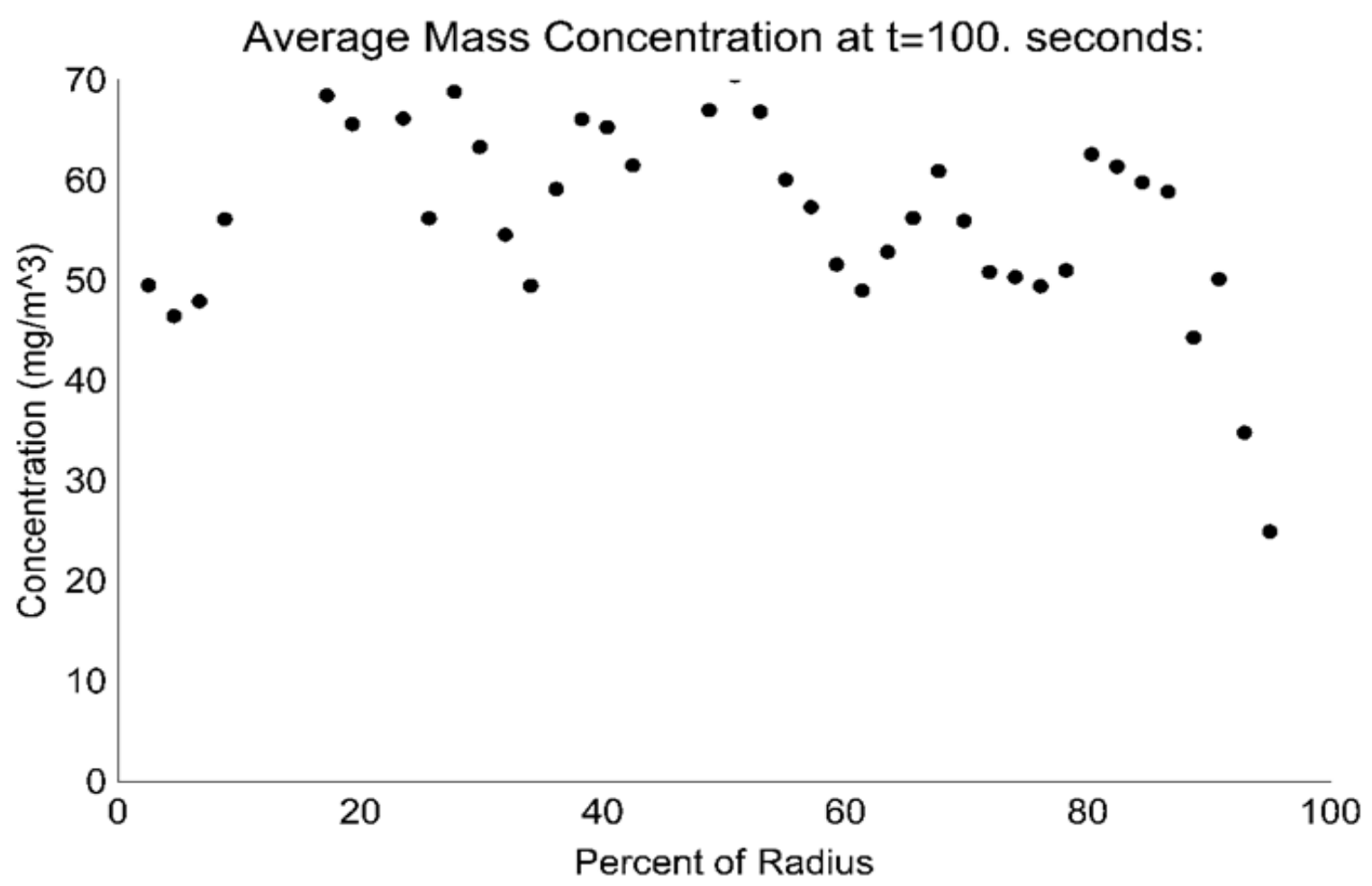

Figure 33: Mass Concentration of Cuboid at 100 Seconds

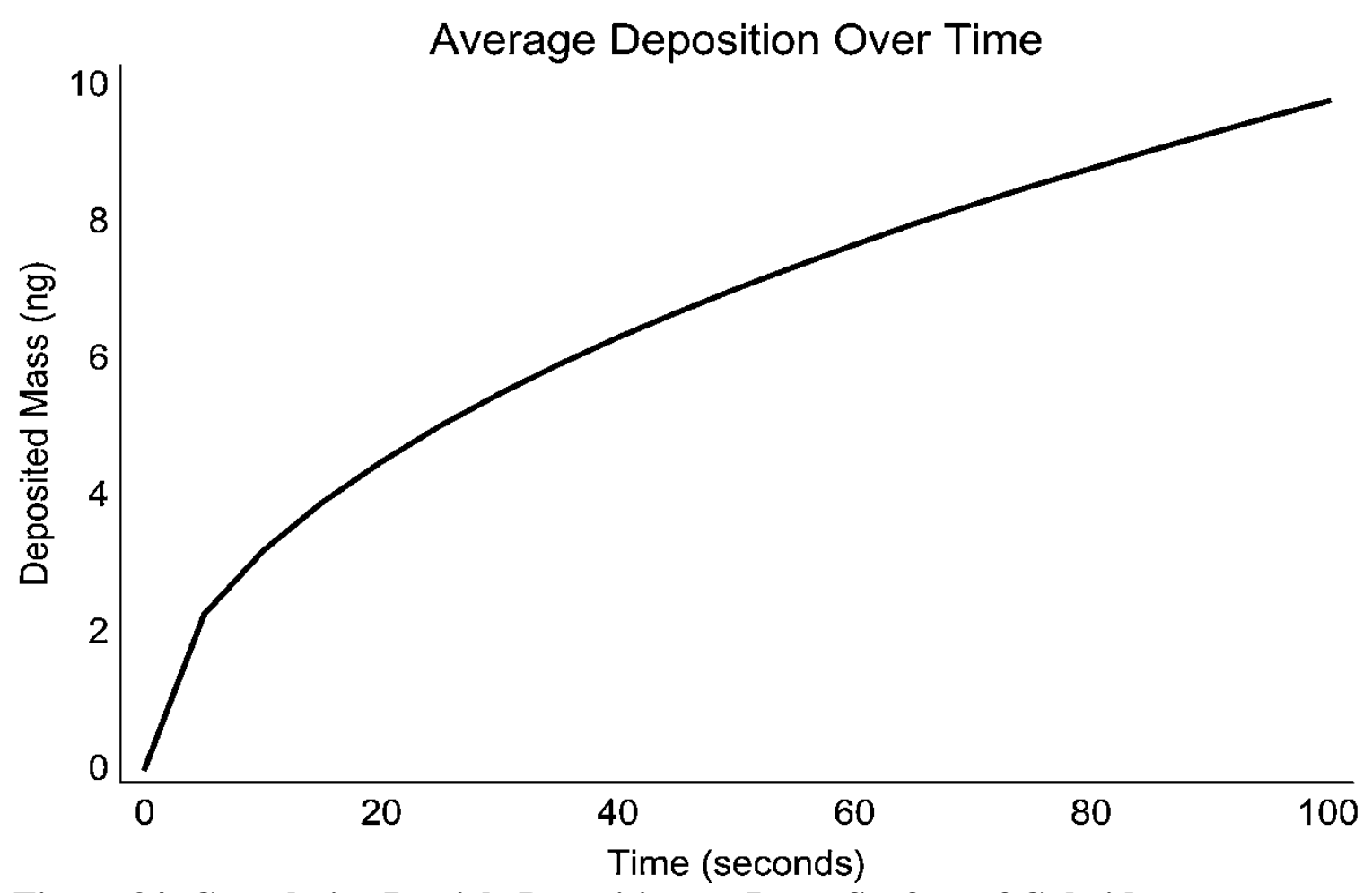

Figure 34: Cumulative Particle Deposition on Inner Surface of Cuboid 
For the cuboid, the cumulative particle deposition over time was also determined. This deposition can be seen in Figure 34, where the cumulative deposition behaves exactly the same as in the preceding geometries. At the beginning of the simulation, the particle deposition climbs quickly at a constant rate. However, after approximately five seconds, the deposition rate starts to slow. By the end of the simulation, approximately 10 ng of aerosol particles have deposited.

\section{Sphere with Internal Obstruction}

The last geometry that was tested in the program was a sphere containing an interior obstruction made from two overlapping miniature spheres. This vessel was used to demonstrate the robustness of the model. As mentioned previously, aerosol particles were allowed to reside inside the sphere but not within the internal obstruction. Figure 35 and Figure 36 correspond to the average mass concentration at $t=50$ seconds and $t=100$ seconds, respectively. To determine average mass concentration, the "Sample Sphere" technique was used along the positive $x$ axis.

In this direction, the spherical obstruction extends a distance of $1.5 R_{\text {Obs }}$ from the origin. Since $R_{\text {Obs }}=R / 4$, the distance can be rewritten in terms of sphere radius $R$ as $3 R / 8$ (or $37.5 \%$ of the total radial distance). Thus, particles in both Figure 35 and Figure 36 should start appearing at $37.5 \%$ of the total distance. However, both figures indicate that the local mass concentration starts increasing slightly before the $37.5 \%$ mark. Fortunately, the reason this occurs is the same reason why it happened to the torus. With the "Sample Sphere" technique, it is possible for a sphere centered just outside a given vessel boundary to have a radius large enough to incorporate some aerosol particles inside the vessel into its calculations. 


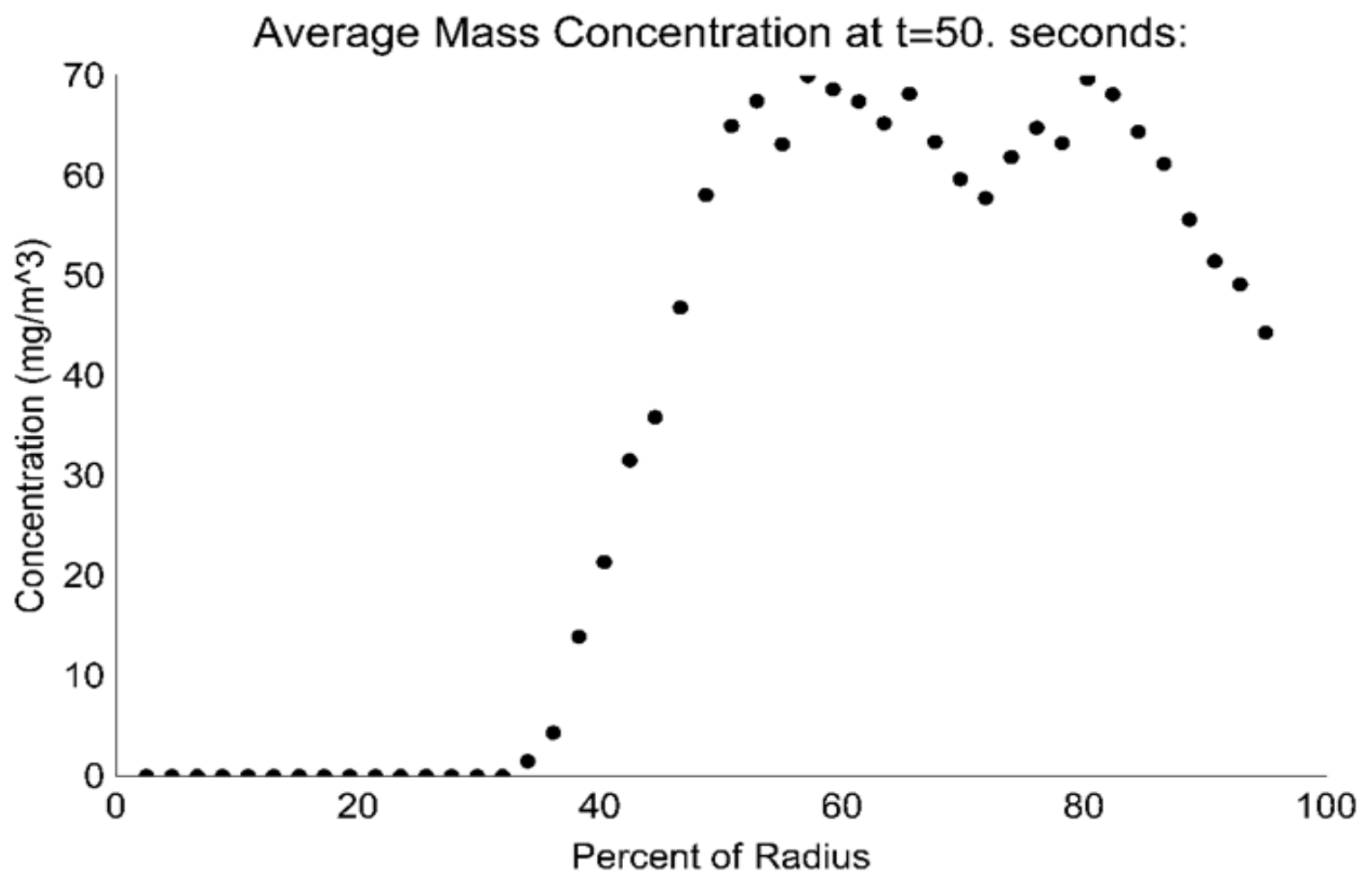

Figure 35: Mass Concentration of Sphere with Internal Obstruction at 50 Seconds

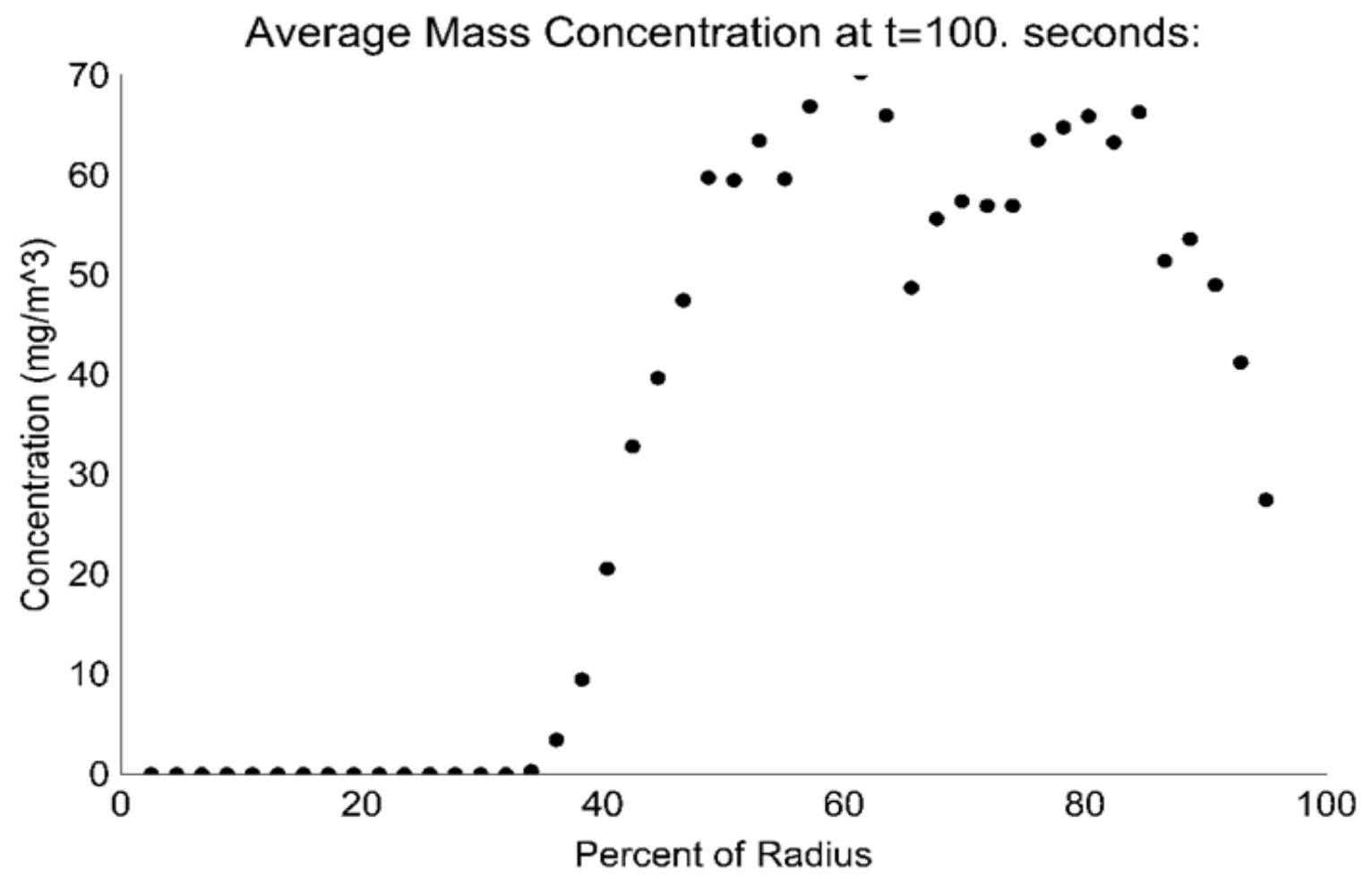

Figure 36: Mass Concentration of Sphere with Internal Obstruction at 100 Seconds 


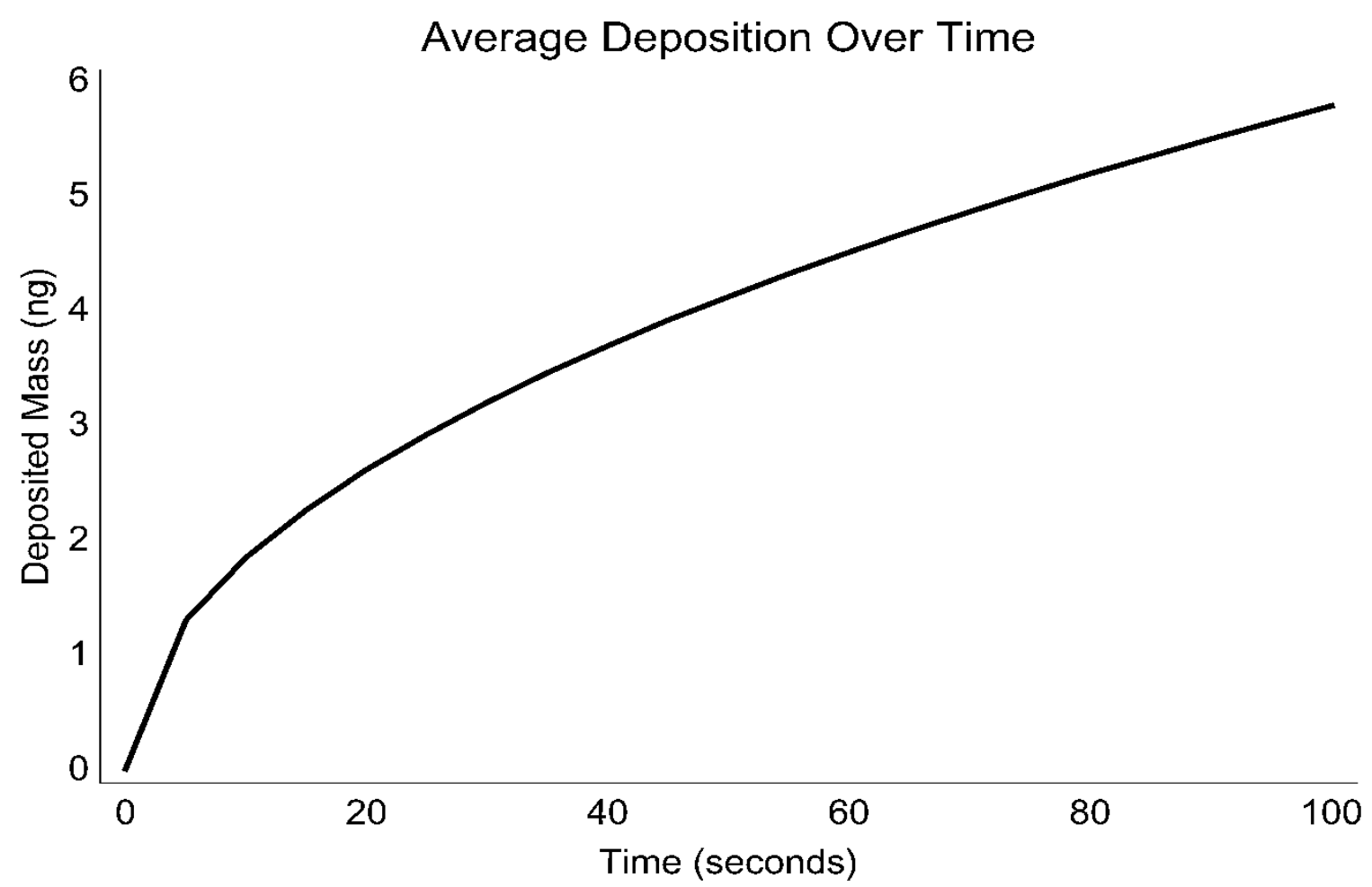

Figure 37: Cumulative Particle Deposition on Inner Surfaces of Sphere with Internal Obstruction

Cumulative particle deposition was also calculated for this vessel, and can be seen in Figure 37. Once again, the cumulative deposition climbs sharply for the first few seconds. The deposition then tapers off in a manner similar to the previous geometries. At the end of the simulation, a little under $6 \mathrm{ng}$ of aerosol particles have deposited. By comparing these results with Figure 22, it is apparent that the addition of the interior obstruction added approximately $0.75 \mathrm{ng}$ of deposited aerosol particles over the life of the simulation. 


\section{RESULTS: DEPOSITON AND COAGULATION}

After modeling the spatially inhomogeneous aerosol model for deposition only, the coagulation phenomenon was incorporated. This was accomplished by changing the value of the coagulation binary switch. Unlike the "Deposition Only" case, which focused on the location of the particles within the sphere, the "Deposition and Coagulation" simulation focused on particle sizes suspended at the given evaluation times. This required creating size "bins," into which the particles could be sorted based on diameter. For the research conducted in this dissertation, 20 size bins were used. The bin boundaries $d_{i}$ were calculated using the equation

$$
d_{i}=d_{\text {Min }} \times\left(\frac{d_{\text {Max }}}{d_{\text {Min }}}\right)^{(i-1) / B} \quad \text { Eq. } 39
$$

where $d_{\text {Min }}$ is the minimum particle diameter, $d_{\text {Max }}$ is the maximum particle diameter, $B$ is the number of size bins, and $i$ runs from one to $i_{M a x}$. Values for these parameters are provided in Table 4.

Table 4: Size Bin Parameters

\begin{tabular}{|l|l|}
\hline Minimum diameter: & $d_{\text {Min }}=0.04 \mu \mathrm{m}$ \\
\hline Maximum diameter: & $d_{M a x}=0.45 \mu \mathrm{m}$ \\
\hline Number of bins: & $B=20$ \\
\hline Number of boundaries: & $i_{M a x}=21$ \\
\hline
\end{tabular}

Parameters for the "Deposition and Coagulation" case are given in the middle column of Table 3. Due to the addition of the second aerosol phenomenon, the total simulated time was reduced from 100 seconds to 20 seconds. The graphical results were generated for each vessel at the halfway mark as well as the simulation end time, 
corresponding to 10 seconds and 20 seconds respectively. Data files were written to the directory every second. Results for each individual geometry are given below.

\section{Sphere}

After adjusting all of the necessary parameters in the aerosol program, the simulation was conducted on an aerosol inside a spherical vessel. This geometry was modeled first in order to establish a benchmark with prior literature published by Campbell et al. ${ }^{33}$ Figure 38 and Figure 39 correspond to the particle size distribution suspended within the sphere at $t=10$ seconds and $t=20$ seconds. In the first figure, the amount of suspended mass peaks at approximately $6.50 \mathrm{ng}$ in the $11^{\text {th }}$ size bin. This corresponds to aerosol particles of $0.134 \mu \mathrm{m}$ to $0.151 \mu \mathrm{m}$ in diameter. Figure 39 , on the other hand, has a suspended mass peak in the $12^{\text {th }}$ size bin, corresponding to aerosol particles $0.151 \mu \mathrm{m}$ to $0.171 \mu \mathrm{m}$ in diameter. Since there are no condensation effects present in this simulation, the peak shift in the two figures must be dependent on the coagulation process in the aerosol model. Another characteristic of the peak in Figure 39 is that it has a value of approximately $6.25 \mathrm{ng}$, which is noticeably less than in the previous figure. This slight decrease in peak height is attributed to particle deposition that occurred between the two evaluation times.

In order to verify the aerosol model, the results for the "Deposition and Coagulation" case had to match results from previously published literature. Fortunately, when Figure 38 and Figure 39 were compared with data provided in the paper by Campbell et al. ${ }^{33}$, the graphs matched perfectly. This indicates that the current aerosol model accurately simulates coagulation phenomenon, and has successfully been combined with the deposition process. 


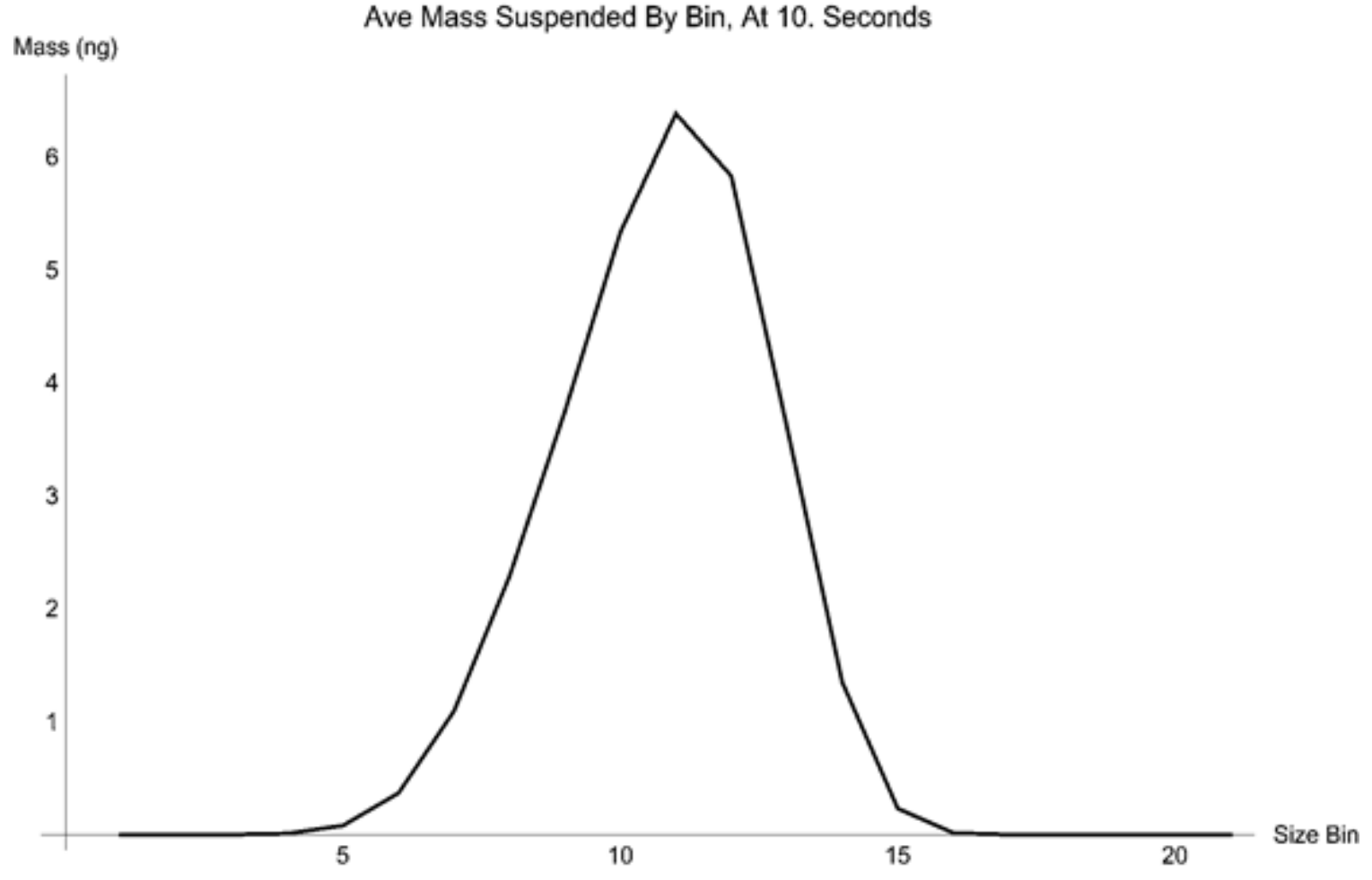

Figure 38: Suspended Mass in Sphere at 10 Seconds

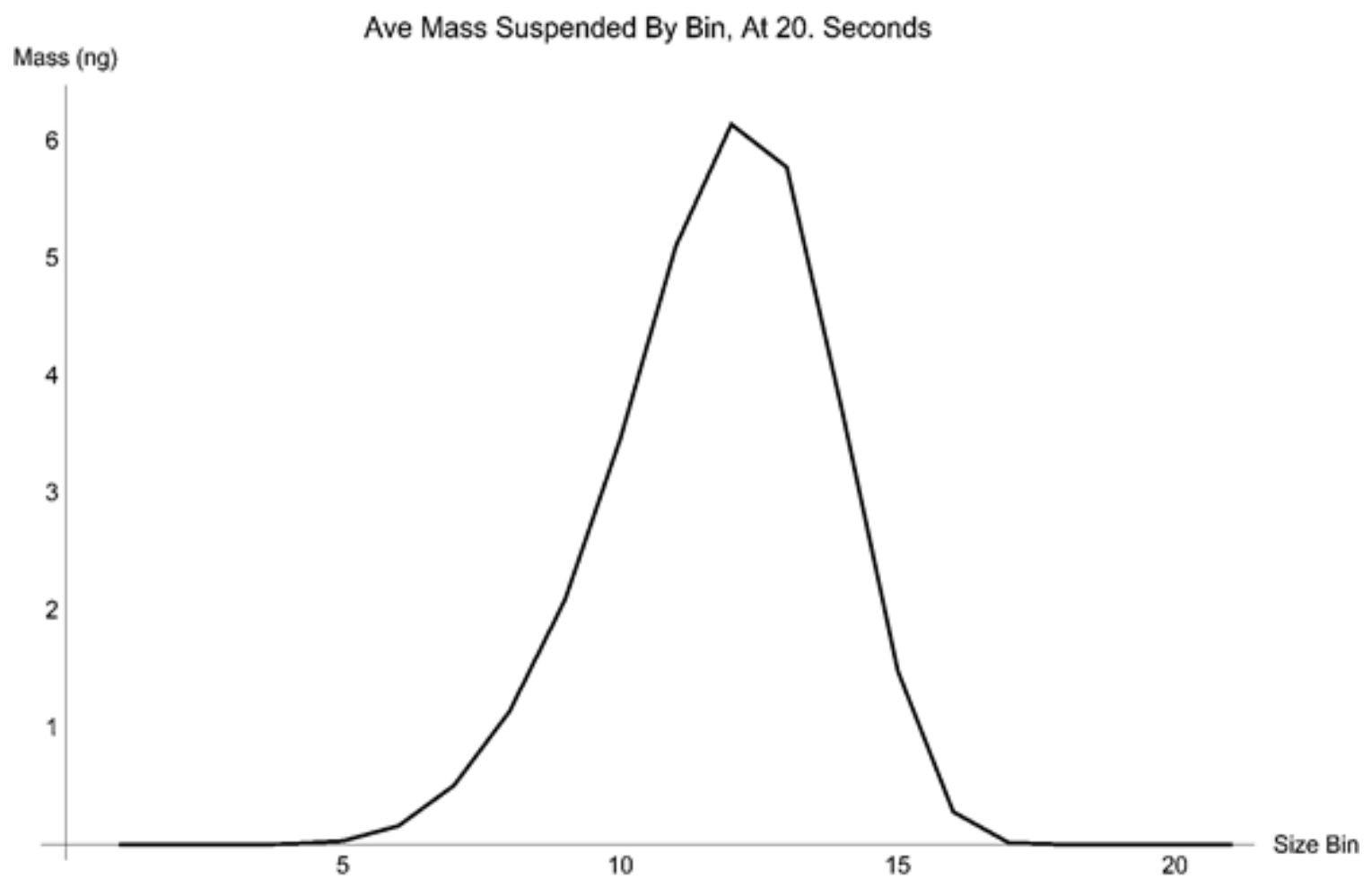

Figure 39: Suspended Mass in Sphere at 20 Seconds 


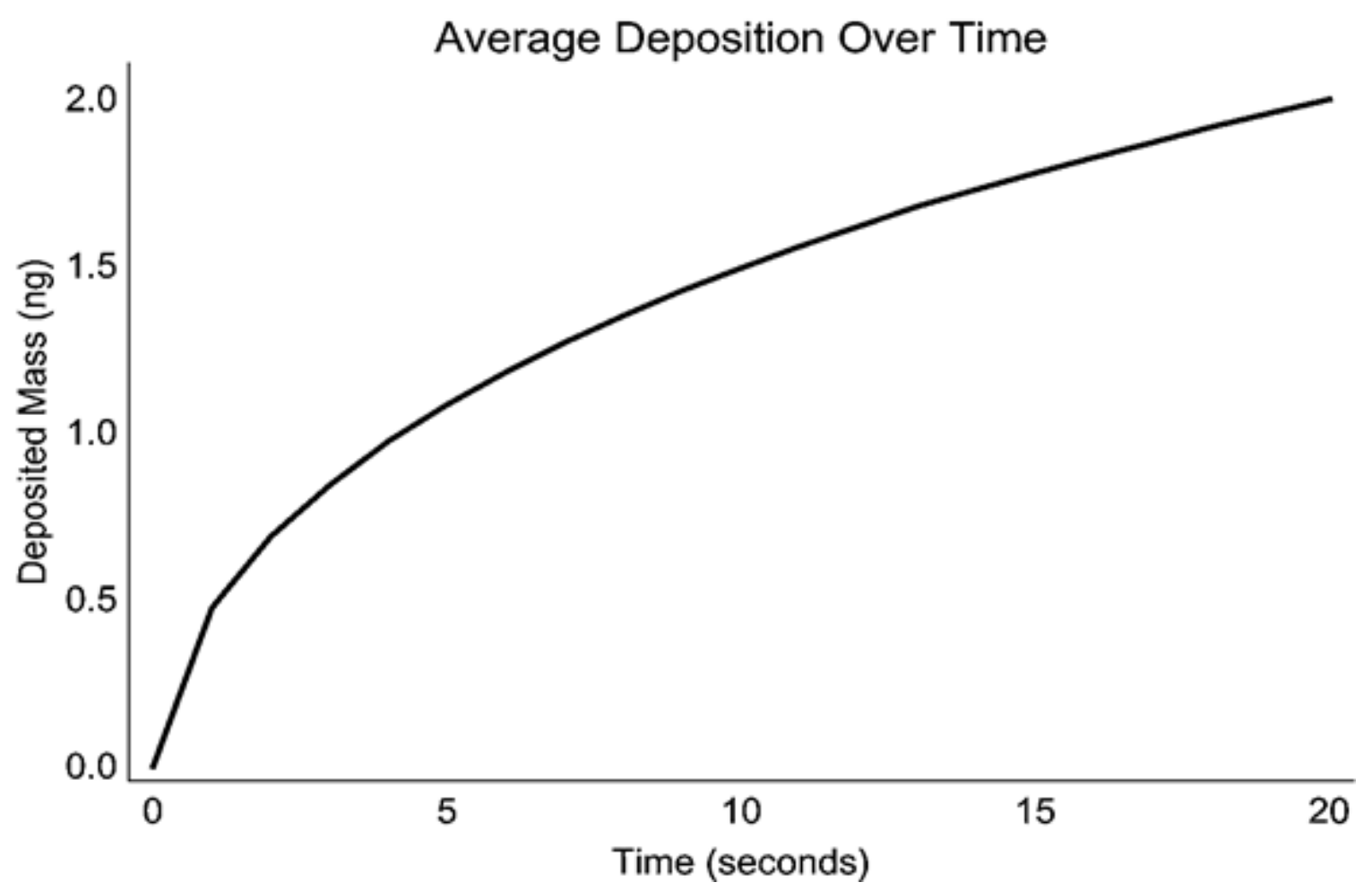

Figure 40: Cumulative Particle Deposition on Inner Surface of Sphere

To determine whether or not the addition of a coagulation component to the model adversely affected the motion of aerosol particles, the cumulative particle deposition on the inner surface of the sphere was plotted again (see Figure 40). As can be seen, as time progresses, the amount of mass deposited follows the same behavior as in the case for deposition only. Further, in the report by Campbell et al. ${ }^{33}$, the deposited mass approached $2 \mathrm{ng}$. This is identical to the value approached in Figure 40. The amount of correlation between the two studies strongly indicates that the spatially inhomogeneous aerosol model used in this work accurately simulates both deposition and coagulation phenomena.

\section{Ellipsoid}

Once the simulation for the spherical vessel was finished, the elliptical geometry was incorporated into the program. As discussed previously, this was due to the 
equational similarity between a sphere and an ellipsoid. Results for the "Deposition and Coagulation" case at $t=10$ seconds and $t=20$ seconds are given in Figure 41 and Figure 42 , respectively. For the first figure, the amount of suspended mass peaks at approximately $2.25 \mathrm{ng}$ in the $11^{\text {th }}$ size bin. This size bin is the same as the one for the spherical vessel at $t=10$ seconds (see Figure 38), and corresponds to aerosol particles of $0.134 \mu \mathrm{m}$ to $0.151 \mu \mathrm{m}$ in diameter. Just like with the previous vessel, the peak at $t=20$ seconds has shifted one to the right, putting it in the $12^{\text {th }}$ size bin. The shifted peak is shown in Figure 42, and corresponds to aerosol particles $0.151 \mu \mathrm{m}$ to $0.171 \mu \mathrm{m}$ in diameter. Due to the lack of condensation, the peak shift can only be from aerosol particle coagulation. Similar to the previous geometry, Figure 42 has a peak value of about $2.10 \mathrm{ng}$, which is less than in the prior figure. This slight decrease in peak height is credited to particle deposition that occurred between the two evaluation times.

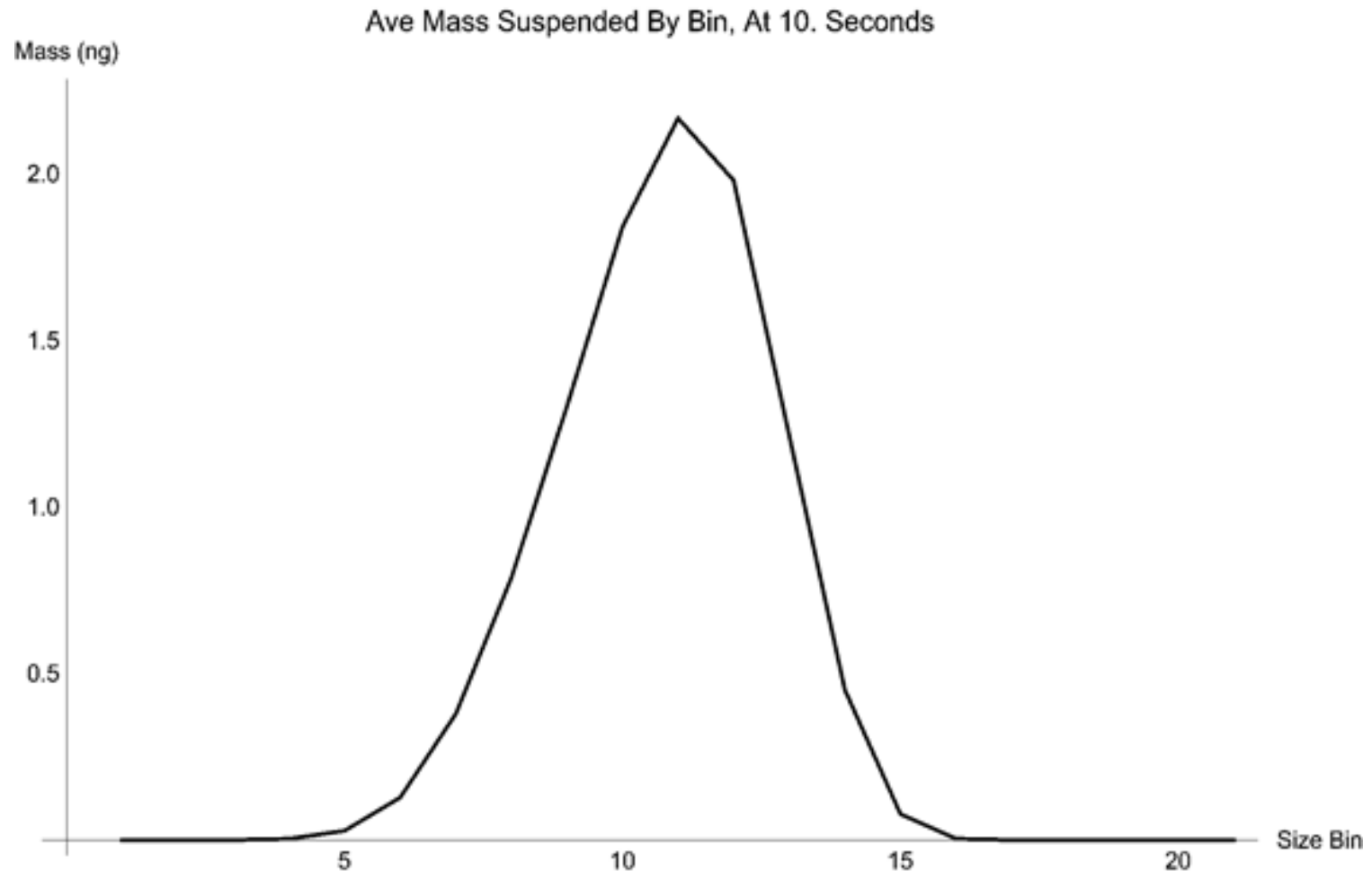

Figure 41: Suspended Mass in Ellipsoid at 10 Seconds 


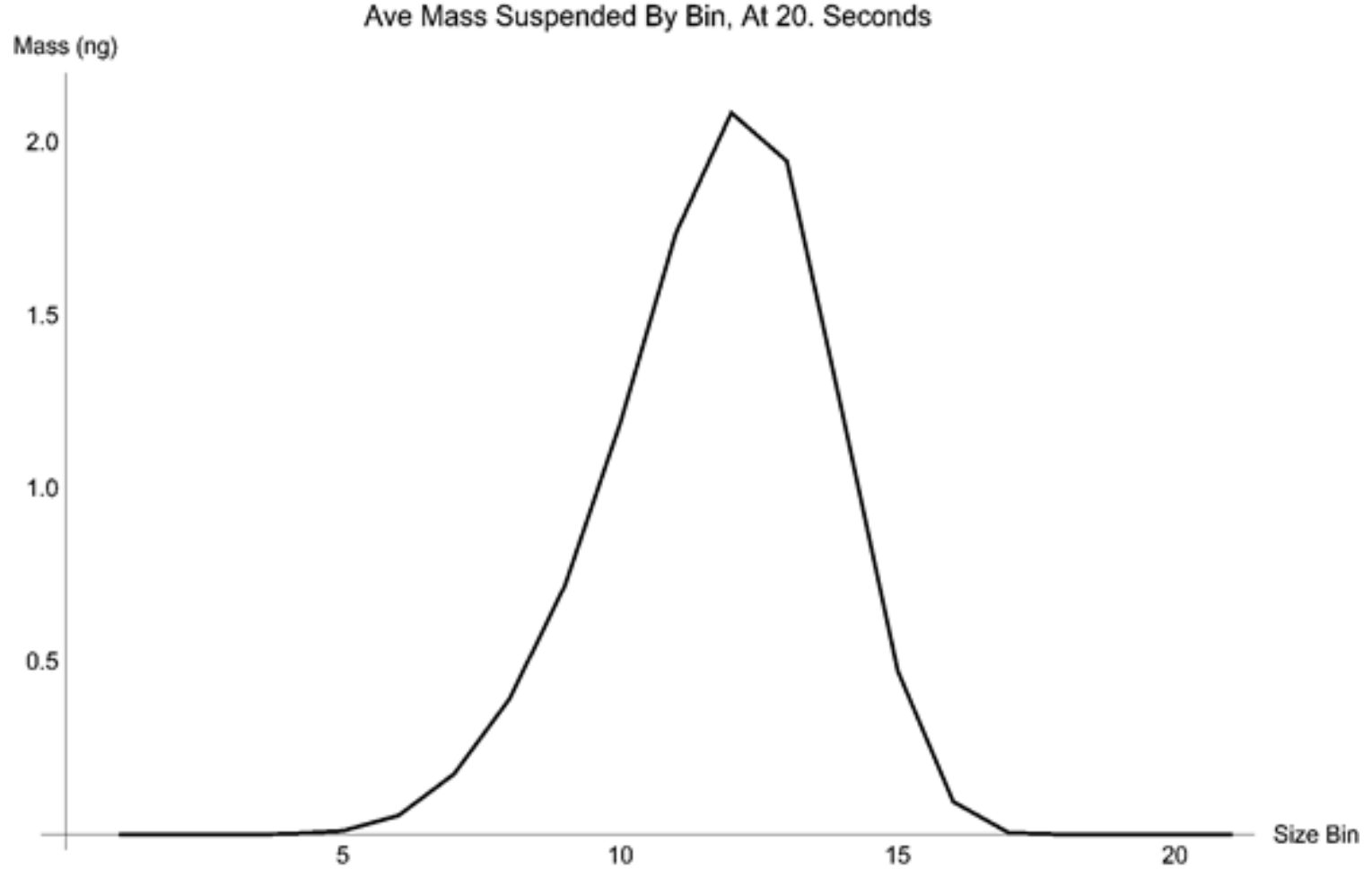

Figure 42: Suspended Mass in Ellipsoid at 20 Seconds

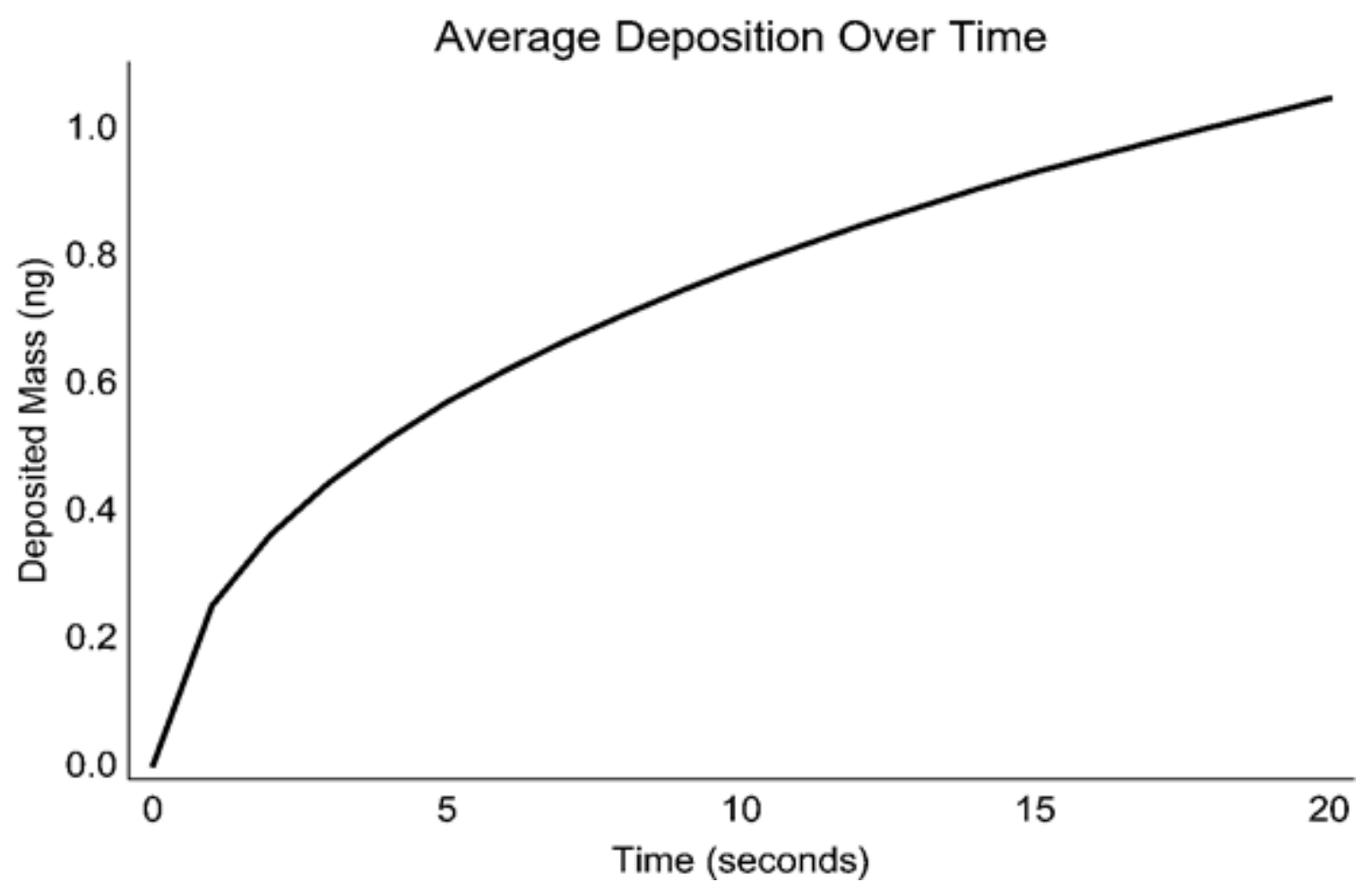

Figure 43: Cumulative Particle Deposition on Inner Surface of Ellipsoid 
Having analyzed the amounts of suspended mass for this vessel, the next step was to determine the cumulative particle deposition over time. This plot is given in Figure 43. From this figure it is apparent that the cumulative deposition climbs sharply for the first second. The deposition rate then tapers off, approaching a value at the end of the simulation of approximately $1 \mathrm{ng}$. This value is approximately half of the amount determined for a spherical vessel in the previous "Deposition and Coagulation" model. However, this difference is attributed to the smaller dimensions provided for the ellipsoid geometry.

\section{Torus}

When the aerosol program finished modeling with the ellipsoid vessel, the torus was incorporated into the simulation. Figure 44 and Figure 45 provide the suspended mass (categorized by size bin) at $t=10$ seconds and $t=20$ seconds, respectively. In keeping with Table 3, both the deposition and coagulation processes were enabled. For the first figure the amount of suspended mass peaks in the $11^{\text {th }}$ size bin $(0.134 \mu \mathrm{m}$ to $0.151 \mu \mathrm{m}$ particle diameter), at a value of approximately $90 \mathrm{ng}$. The relatively large value of the peak is credited to the large spatial dimensions of the vessel. Fortunately, these dimensions don't affect aerosol behavior. Just like with the previous two vessels, the peak at $t=20$ seconds has shifted to the $12^{\text {th }}$ size bin. The shifted peak is shown in Figure 45, corresponding to aerosol particles $0.151 \mu \mathrm{m}$ to $0.171 \mu \mathrm{m}$ in diameter. Again, since no condensation takes place, the peak shift can only from aerosol particle coagulation. While the slight decrease in peak height does occur here, the approximate magnitude of that change is difficult to determine. This difficulty is due to the fixed resolution of the $y$ axis. 


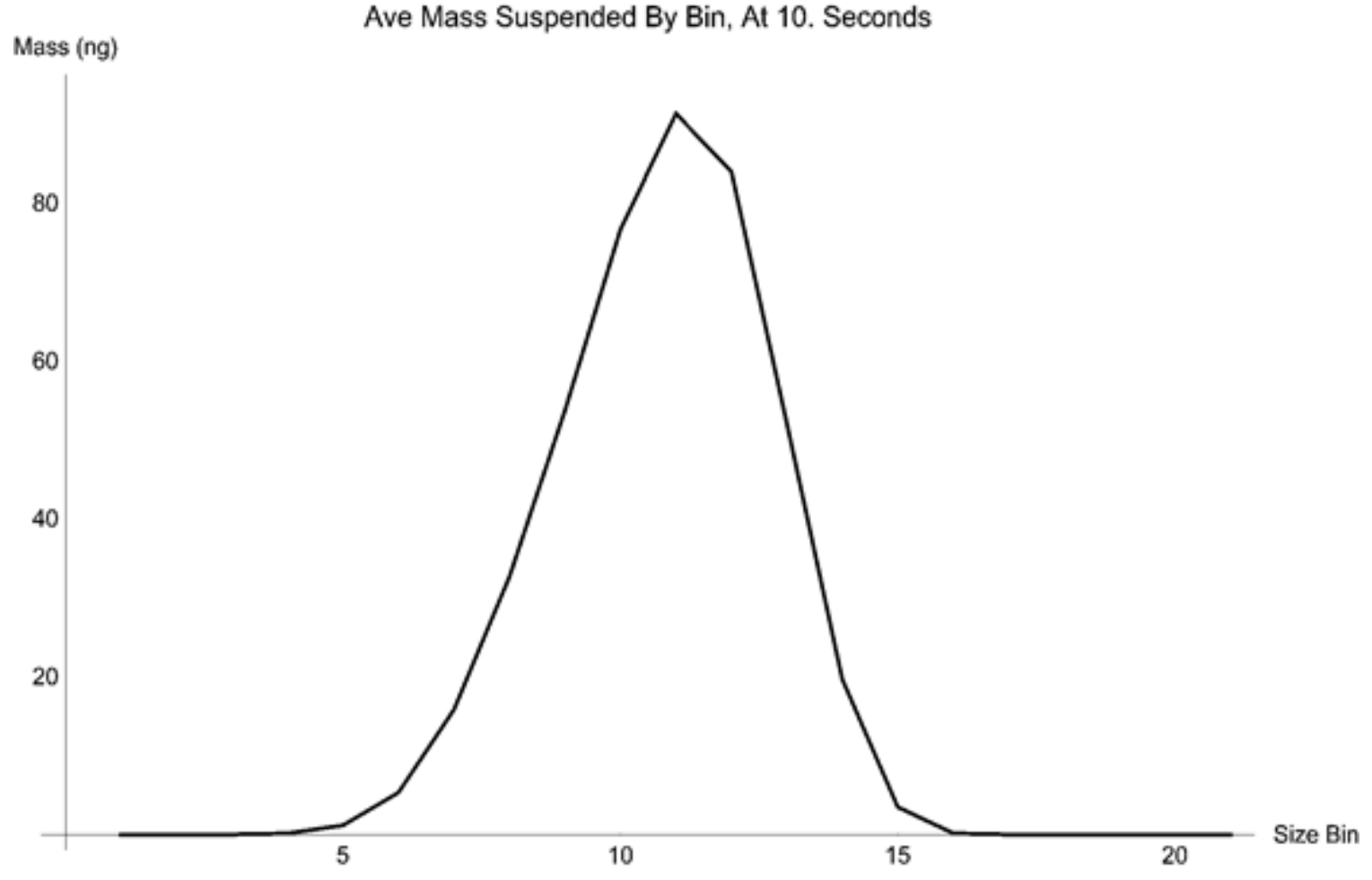

Figure 44: Suspended Mass in Torus at 10 Seconds

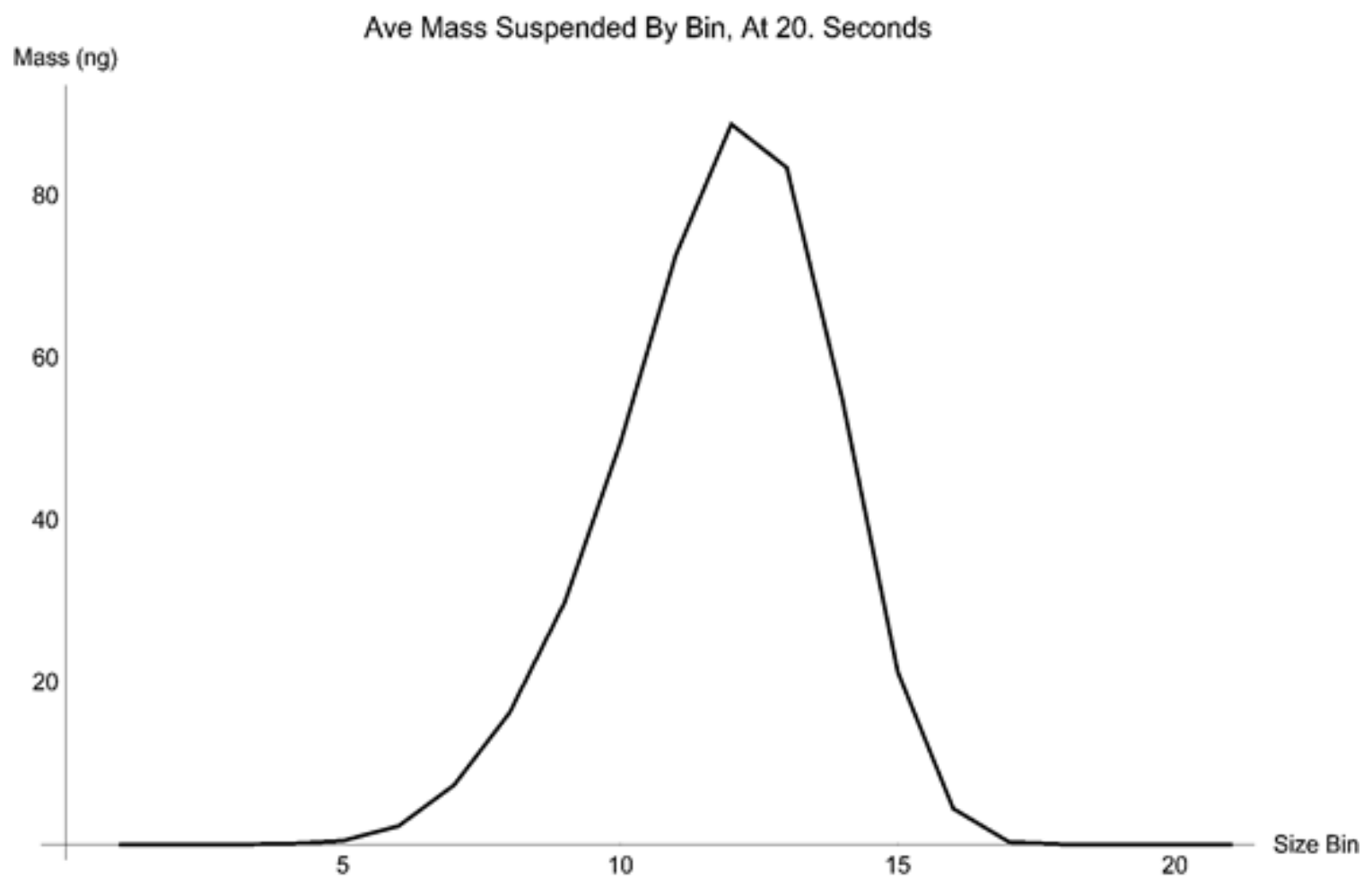

Figure 45: Suspended Mass in Torus at 20 Seconds 


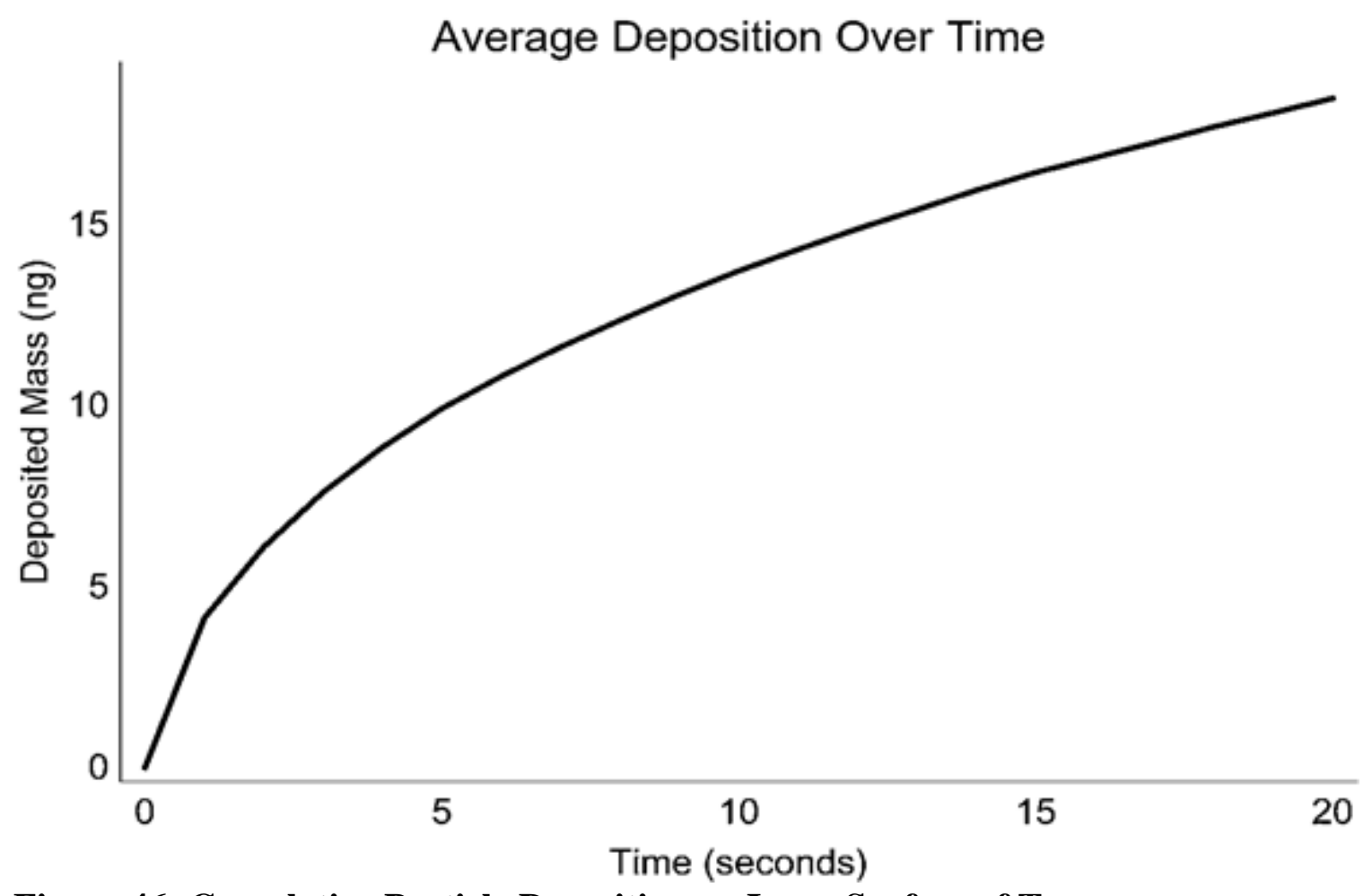

Figure 46: Cumulative Particle Deposition on Inner Surface of Torus

For the torus, the cumulative particle deposition over time was also determined. This deposition can be seen in Figure 46, where the cumulative deposition behaves exactly the same as in the preceding geometries. At the beginning of the simulation, the particle deposition climbs quickly at a constant rate. However, after approximately one second, the deposition rate starts to slow. By the end of the simulation, approximately $17.5 \mathrm{ng}$ of aerosol particles have deposited.

\section{Elliptical Cylinder}

After working with the toroidal vessel, the elliptical cylinder was selected as the next geometry to be used in the program. The average suspended mass was categorized by size bin at $t=10$ seconds and $t=20$ seconds, and the results are given in Figure 47 and Figure 48, respectively. In the figure corresponding to $t=10$ seconds, the amount of suspended mass peaks in the $11^{\text {th }}$ size bin at a value of approximately $7.00 \mathrm{ng}$. For the 
second figure (corresponding to $t=20$ seconds), the peak has a value of around $6.75 \mathrm{ng}$ and has shifted to the right by one size bin. Due to the lack of condensation processes active in the model, the peak shift is attributed to aerosol particle coagulation. The slight decrease in peak height is credited to particle deposition that occurred between the two evaluation times.

The cumulative particle deposition over time was also calculated for this vessel, and can be seen in Figure 49. Just like with previous geometries, the cumulative deposition climbs quickly for the first $5 \%$ of the simulation. Then the deposition rate slows down, causing the overall deposition to taper off in a manner similar to the prior vessels. At the end of the simulation, nearly $2.75 \mathrm{ng}$ of aerosol particles have deposited on the inside of the elliptical cylinder.

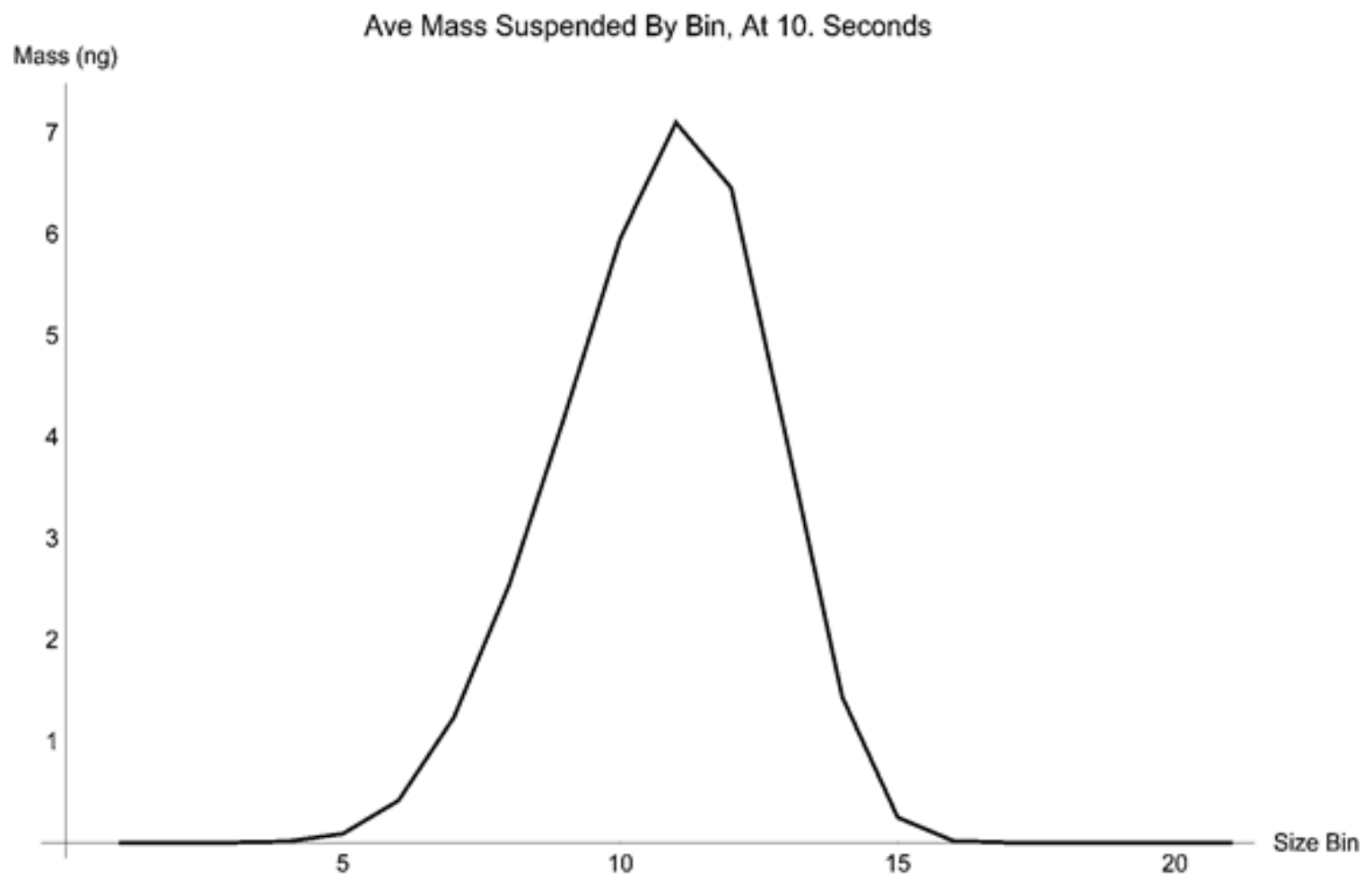

Figure 47: Suspended Mass in Cylinder at 10 Seconds 


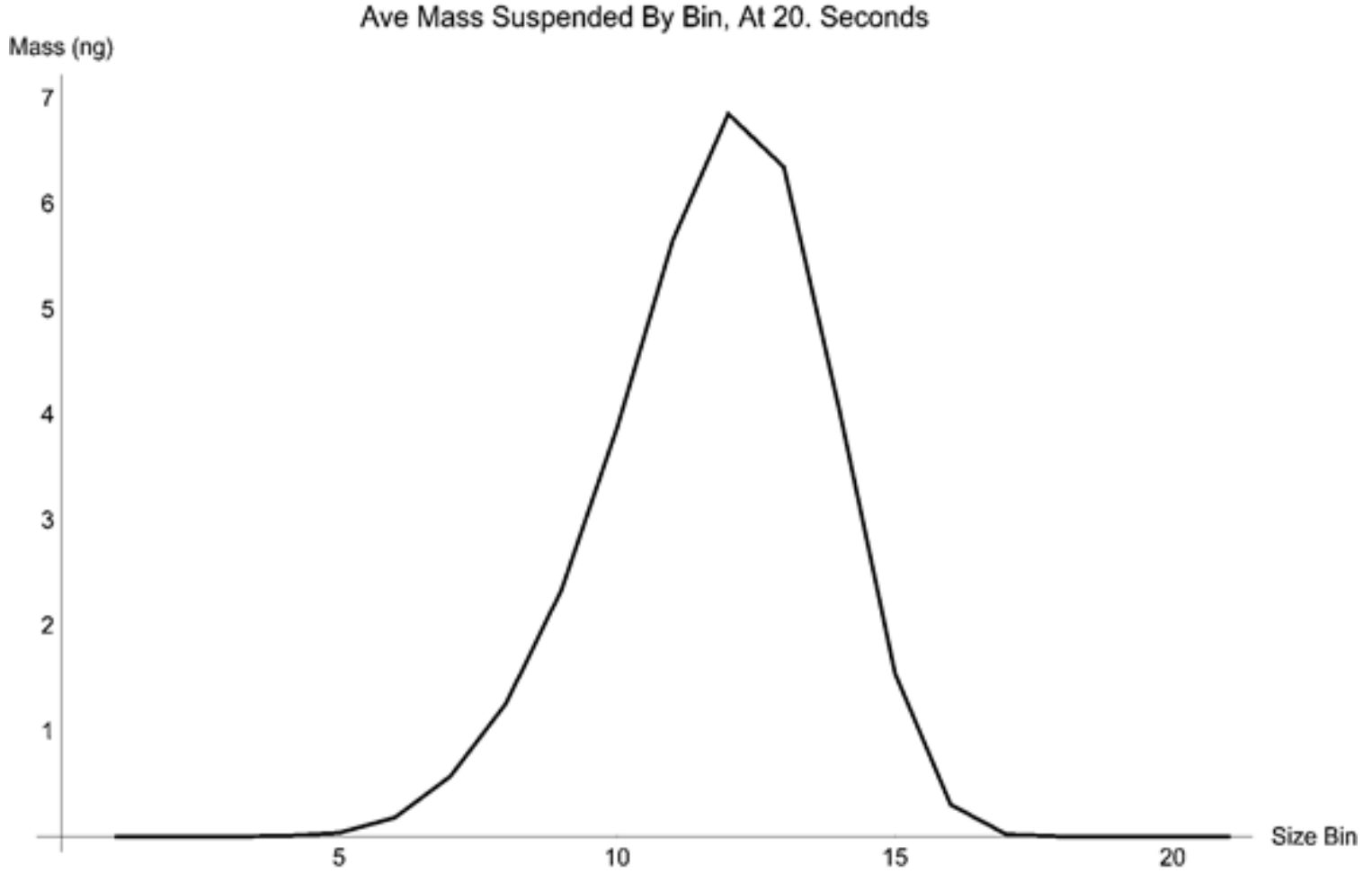

Figure 48: Suspended Mass in Cylinder at 20 Seconds

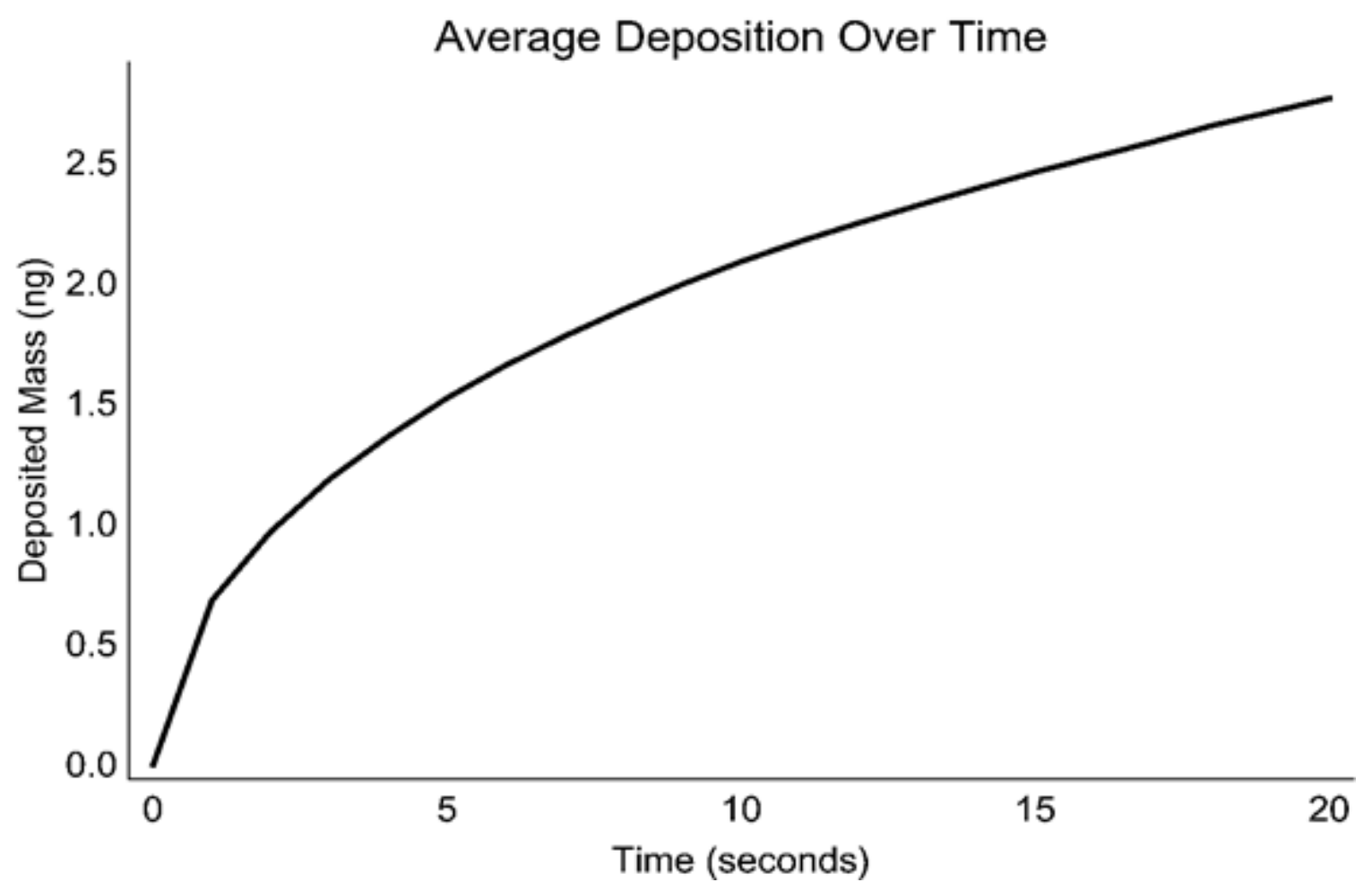

Figure 49: Cumulative Particle Deposition on Inner Surface of Cylinder 


\section{Cuboid}

Once the simulations had been completed for the elliptical cylinder, vessel modeling was extended to the cuboid geometry. This was because it provided the opportunity to increase the number of physical discontinuities that needed to be modeled in the program. Figure 50 and Figure 51 give the average suspended mass for the cuboid at 10 and 20 seconds into the simulation. Just like with previous geometries, the first figure peaks at the $11^{\text {th }}$ size bin, while the latter figure's peak corresponds to the $12^{\text {th }}$ size bin. For Figure 50, the peak suspended mass is approximately $12.00 \mathrm{ng}$. In the next graph (Figure 51), this peak appears to have decreased in magnitude to a value near $11.75 \mathrm{ng}$. As mentioned in the analysis of previous vessels for the "Deposition and Coagulation" case, no condensation process is being used. Thus, the peak shift in these two figures is due to the coagulation of aerosol particles.

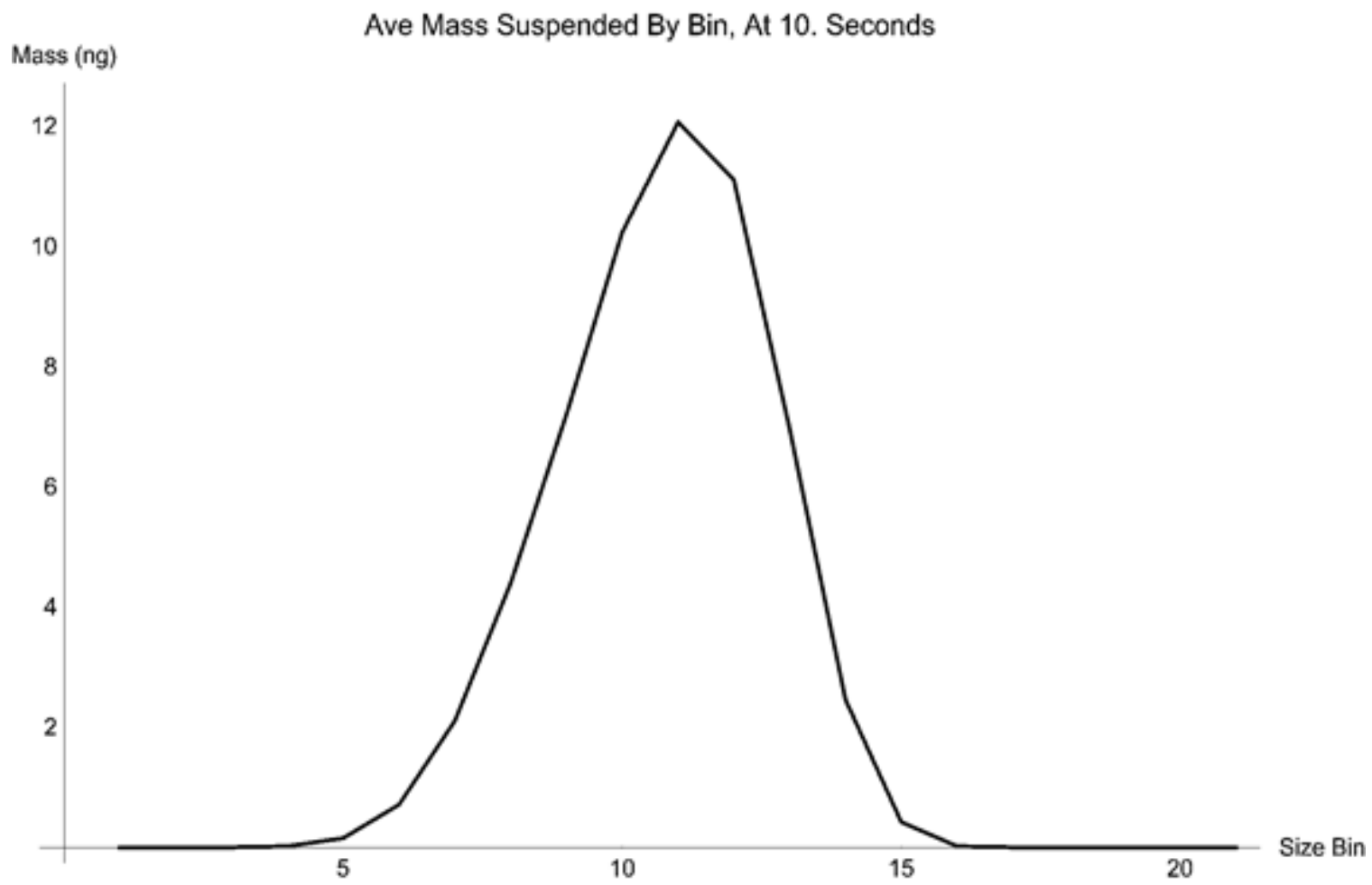

Figure 50: Suspended Mass in Cuboid at 10 Seconds 


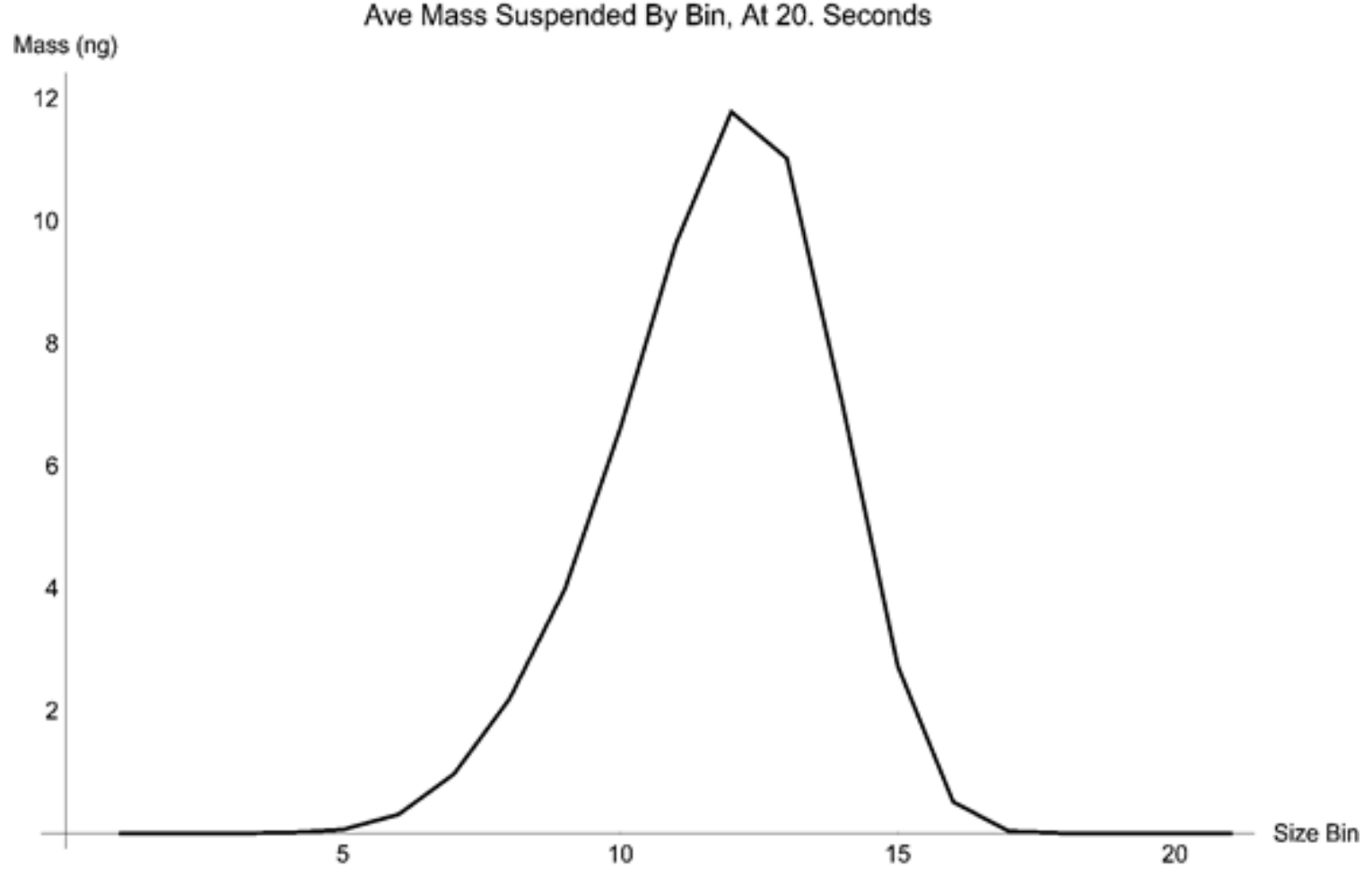

Figure 51: Suspended Mass in Cuboid at 20 Seconds

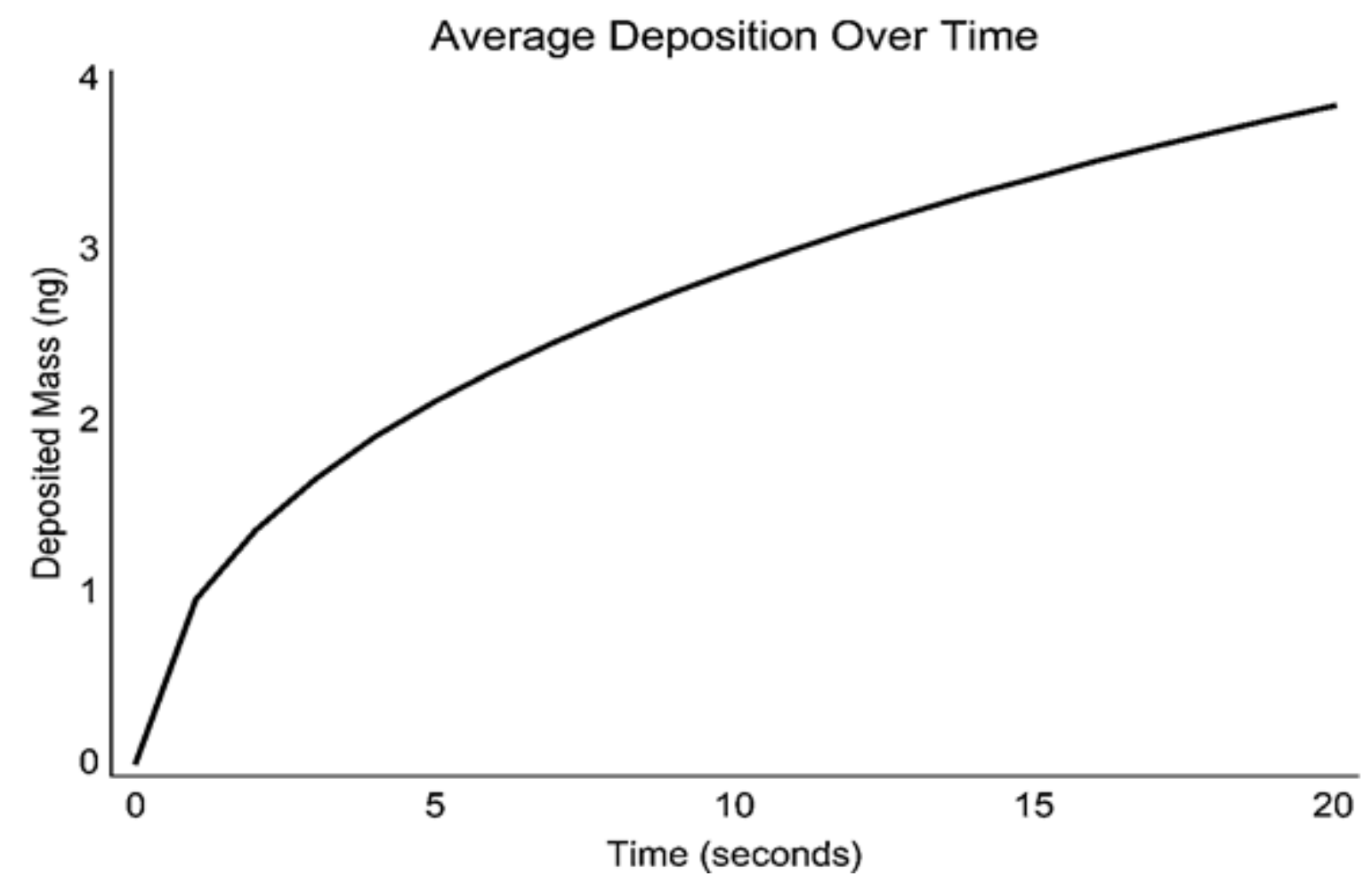

Figure 52: Cumulative Particle Deposition on Inner Surface of Cuboid 
Cumulative particle deposition was also calculated for the cuboid, and can be seen in Figure 52. Once again, the cumulative deposition climbs sharply for the first second. This deposition then levels off as time approaches $t=20$ seconds. As discussed previously, the general deposition behavior mentioned here is mirrored repeatedly in the prior vessels. At the end of the simulation, approximately $3.75 \mathrm{ng}$ of aerosol particles have deposited on the interior of the cuboid. This value is the same order of magnitude as both the sphere and ellipsoid (which user similar dimensions), and so the total deposition amount is considered reasonable for the geometry.

\section{Sphere with Internal Obstruction}

After all the fundamental vessel geometries had been modeled, the spherical vessel containing an interior obstruction was incorporated into the program. This obstruction was made from two overlapping miniature spheres. Aerosol particles were only allowed to reside in the space between the outer sphere and the internal obstruction. The average suspended masses for this shape at $t=10$ seconds and $t=20$ seconds are given in Figure 53 and Figure 54, respectively. In the first figure the amount of suspended mass peaks in the $11^{\text {th }}$ size bin, corresponding to particles $0.134 \mu \mathrm{m}$ to 0.151 $\mu \mathrm{m}$ in diameter. The magnitude of the peak is a little over $6.00 \mathrm{ng}$. For the second figure the peak has shifted to the right by one size bin, corresponding to aerosol particles 0.151 $\mu \mathrm{m}$ to $0.171 \mu \mathrm{m}$ in diameter. In this graph, the peak's value is approximately $5.80 \mathrm{ng}$. Due to the lack of condensation processes active in the model, the peak's shift to the right is attributed to aerosol particle coagulation. The slight decrease in peak height is credited to particle deposition that occurred between the two evaluation times. 


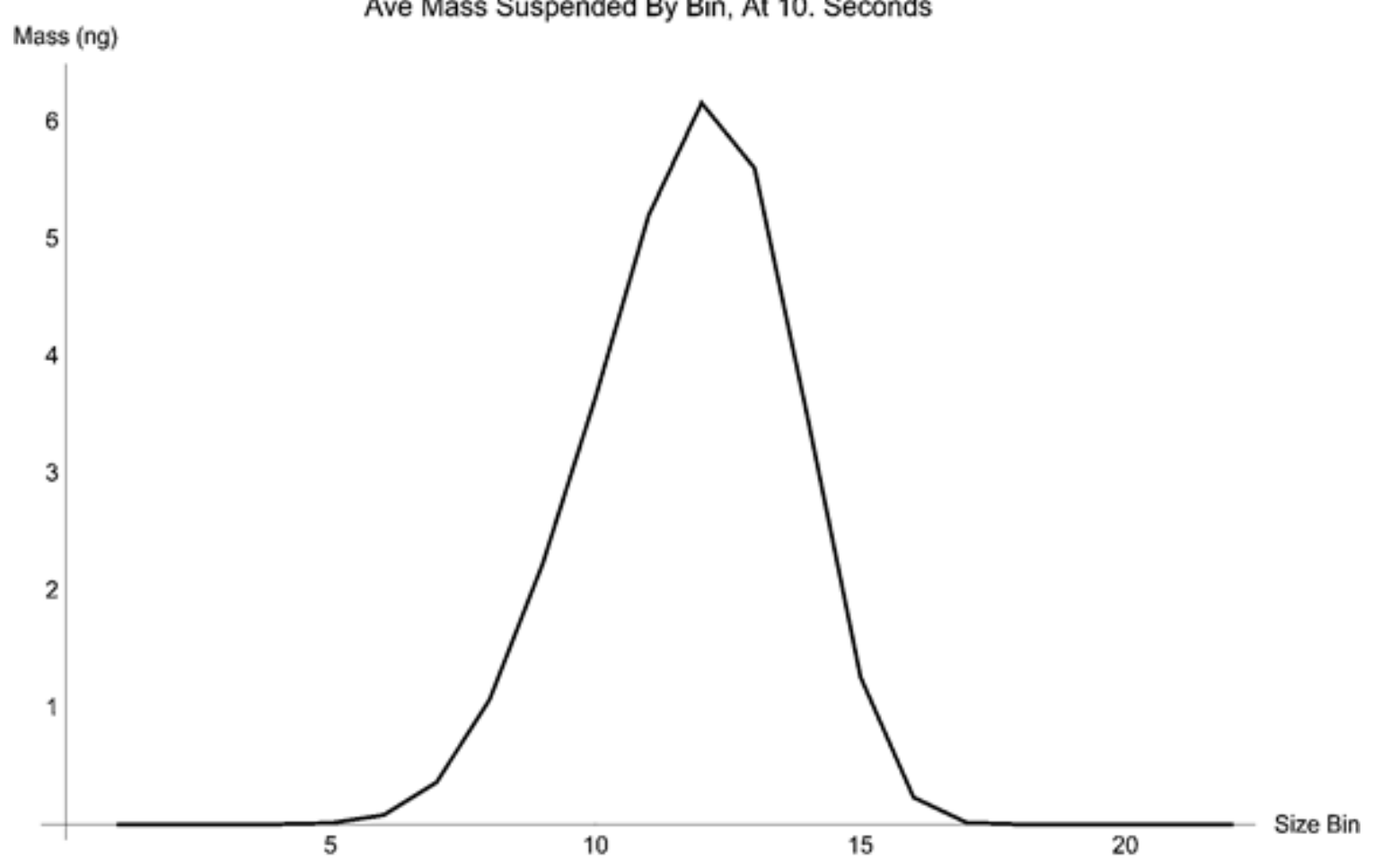

Figure 53: Suspended Mass in Sphere with Internal Obstruction at 10 Seconds

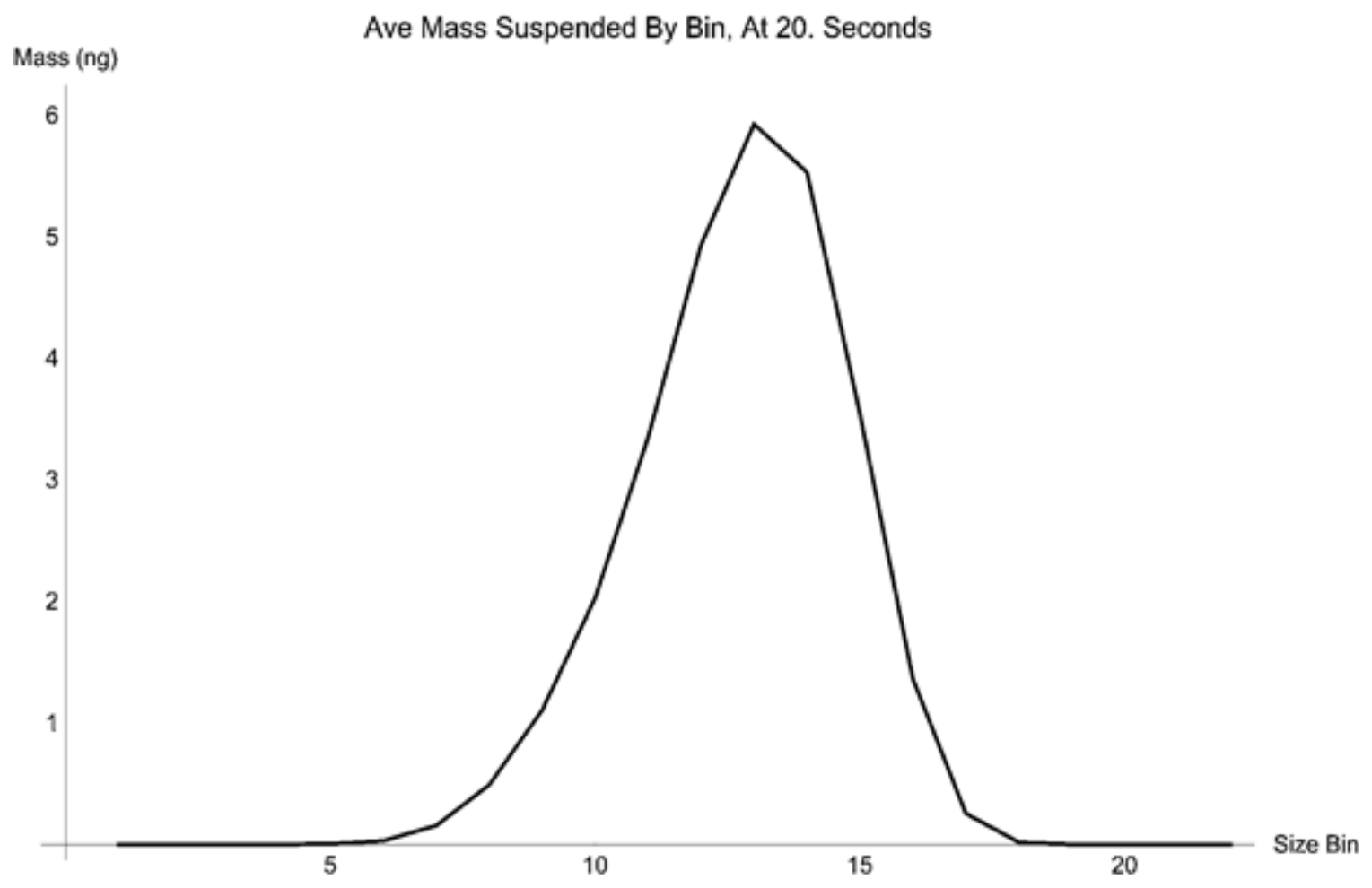

Figure 54: Suspended Mass in Sphere with Internal Obstruction at 20 Seconds 


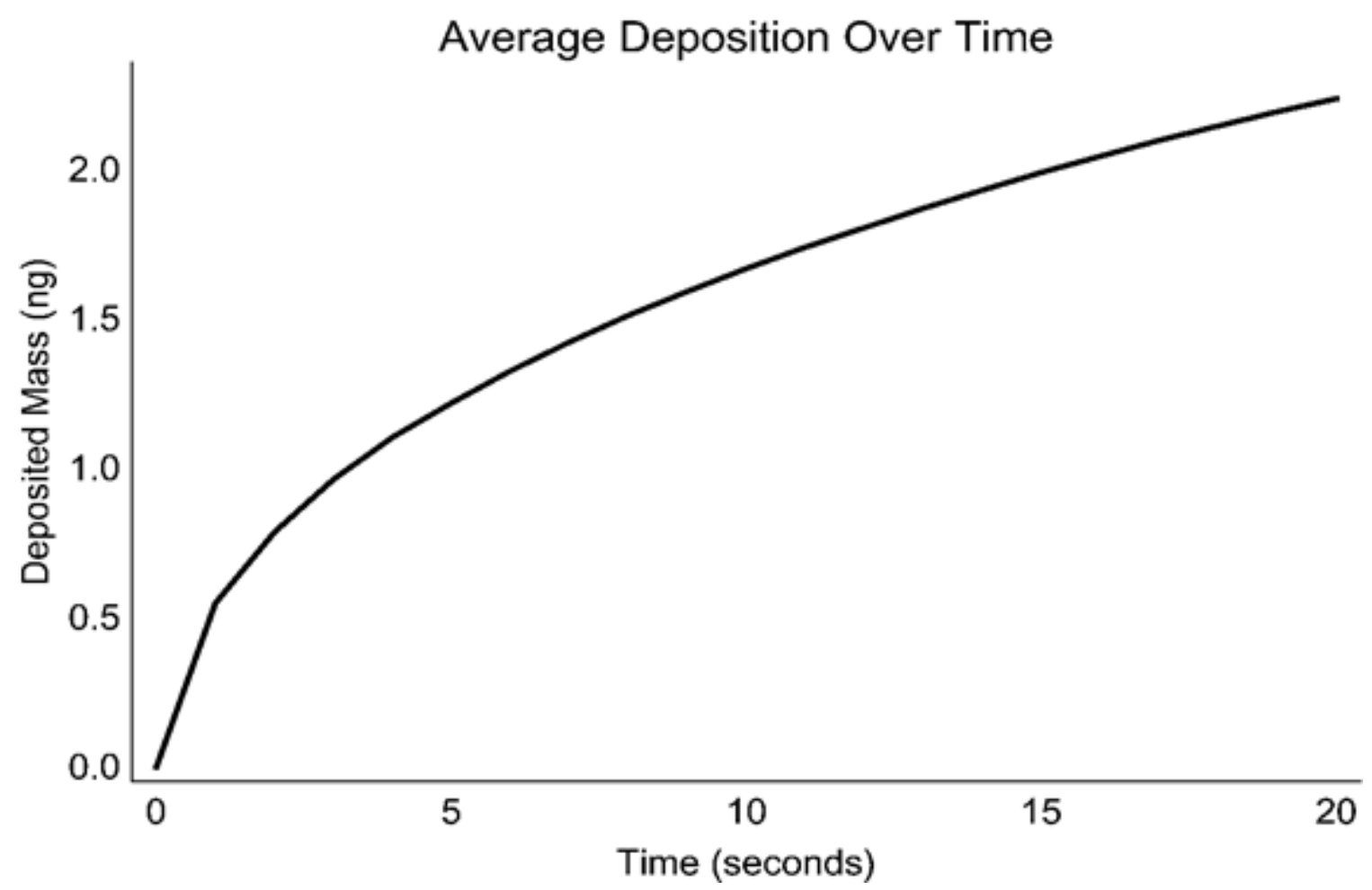

Figure 55: Cumulative Particle Deposition on Inner Surfaces of Sphere with Internal Obstruction

In addition to the local mass concentration, the cumulative particle deposition was also calculated. Total deposition over time for the vessel is shown in Figure 55. As can be seen, the cumulative deposition behaves exactly the same as for previous vessels. The identical deposition behavior across all vessel geometries strongly suggests that the general behavior does not rely on the vessel shape. However, the deposition magnitude is dependent on the shape's dimensions (and the total surface inside the vessel). Indeed, for the spherical vessel with an internal obstruction, well over $2 \mathrm{ng}$ of aerosol particles deposited during the life of the simulation. Comparing these results with Figure 40, it is apparent that the addition of the interior obstruction increased the total amount of aerosol particles that were deposited. 


\section{RESULTS: DEPOSITON, COAGULATION, AND CONDENSATION}

Once all the simulations for the "Deposition and Coagulation" case had been completed, the condensation process was incorporated into the spatially inhomogeneous aerosol model. This was accomplished by simply changing the value of the text-based binary switch corresponding to the condensation process, as described in Chapter V. Parameters for this final case are given in the right column of Table 3. The addition of the condensation phenomenon in the program increased computational complexity, so the total simulated time was kept at a total of 20 seconds. Just like with the preceding simulation case, the graphical results were generated for each vessel at the halfway mark as well as the simulation end time, corresponding to 10 seconds and 20 seconds respectively. Data files were written to the directory every second.

Due to the increased computational complexity of the deposition, coagulation, and condensation aerosol model, only a few geometries were evaluated. These shapes are the sphere, cuboid, and sphere with internal obstruction. In order to see the how the addition of the condensation process affects the model, both the local mass concentration and the suspended particle size distribution for each vessel were plotted again. However, unlike the previous two chapters, the cumulative deposition over time is not considered. Instead, the cumulative amount of condensation over time was determined. Results for each of the vessels are given below.

\section{Sphere}

The first vessel geometry modeled with all three aerosol processes was a sphere. At $t=10$ seconds and $t=20$ seconds, the average mass concentration was calculated. 
The results are given in Figure 58 and Figure 59. In keeping with other concentration calculations, the "Sample Sphere" technique was used along an arbitrary axis. Unlike all the previous spatially inhomogeneous aerosol simulations, the concentration actually grows with time. After $t=10$ seconds, the mass concentration oscillates around a value of $500 \mathrm{mg} / \mathrm{m}^{3}$. By $t=20$ seconds, this average value has grown to approximately 1300 $\mathrm{mg} / \mathrm{m}^{3}$. This large growth is due to the condensation in the aerosol model.

In order to further recognize the significance of the condensation effect for the simulation, the two mass concentration graphs can be compared with the data provided in the "Deposition Only" case for the same geometry (see Figure 19 and Figure 21). Although run for five times longer, the mass concentration in these figures maintains a value of approximately $60 \mathrm{mg} / \mathrm{m}^{3}$. From the difference in concentrations it is apparent that the condensation process speeds up particle growth by orders of magnitude.

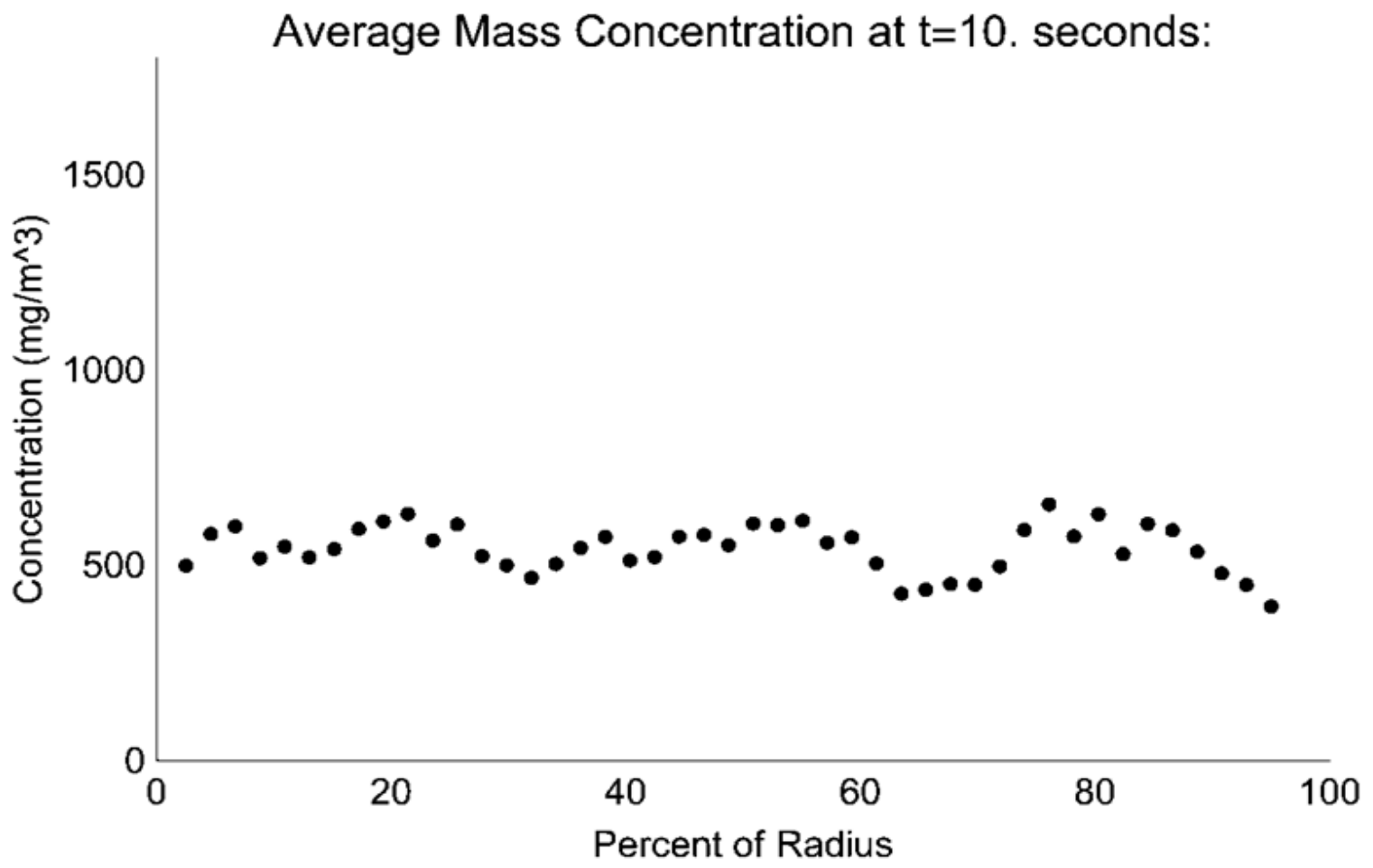

Figure 56: Mass Concentration of Sphere at 10 Seconds 


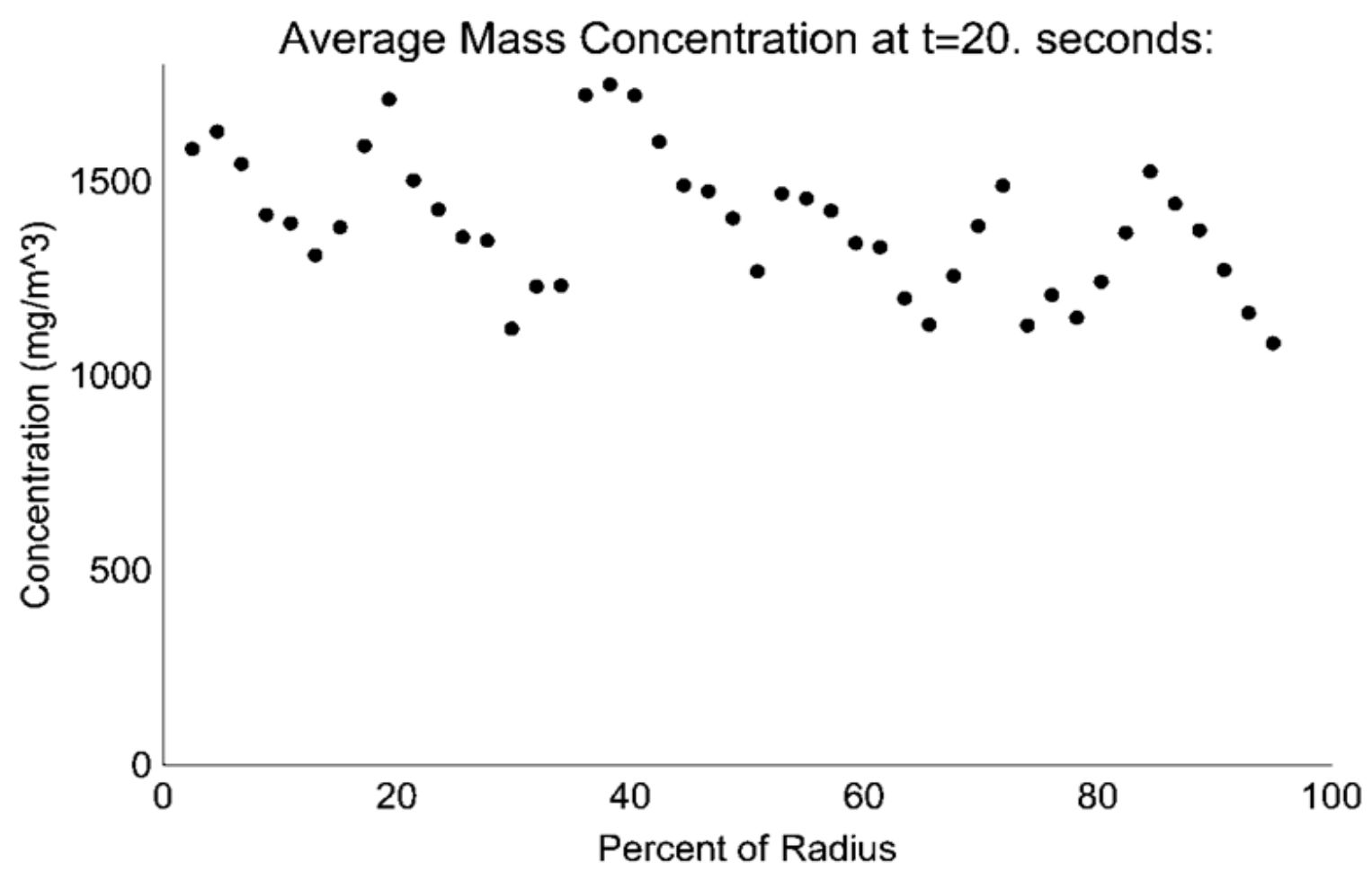

Figure 57: Mass Concentration of Sphere at 20 Seconds

Figure 58 and Figure 59 correspond to the particle size distribution suspended within the sphere at $t=10$ seconds and $t=20$ seconds. In the first figure, the amount of suspended mass peaks at approximately $90 \mathrm{ng}$ in the $16^{\text {th }}$ size bin. This corresponds to aerosol particles of $0.246 \mu \mathrm{m}$ to $0.277 \mu \mathrm{m}$ in diameter. Figure 59 , on the other hand, has a suspended mass peak in the $20^{\text {th }}$ size bin, corresponding to aerosol particles $0.399 \mu \mathrm{m}$ to $0.450 \mu \mathrm{m}$ in diameter. The total overall shift is four size bins, three more than in the "Deposition and Coagulation" case for the same time duration. Since no other parameters have been changed, the peak shift in the two figures must be dependent on the condensation effects present in this simulation. Interestingly, this peak shift is so strong that a portion of the particles in Figure 59 have actually exceeded the maximum size bin allowed for the program. This is why a portion of the line in the graph is missing. To resolve this, the maximum size bin allowed in the program would need to be adjusted. 


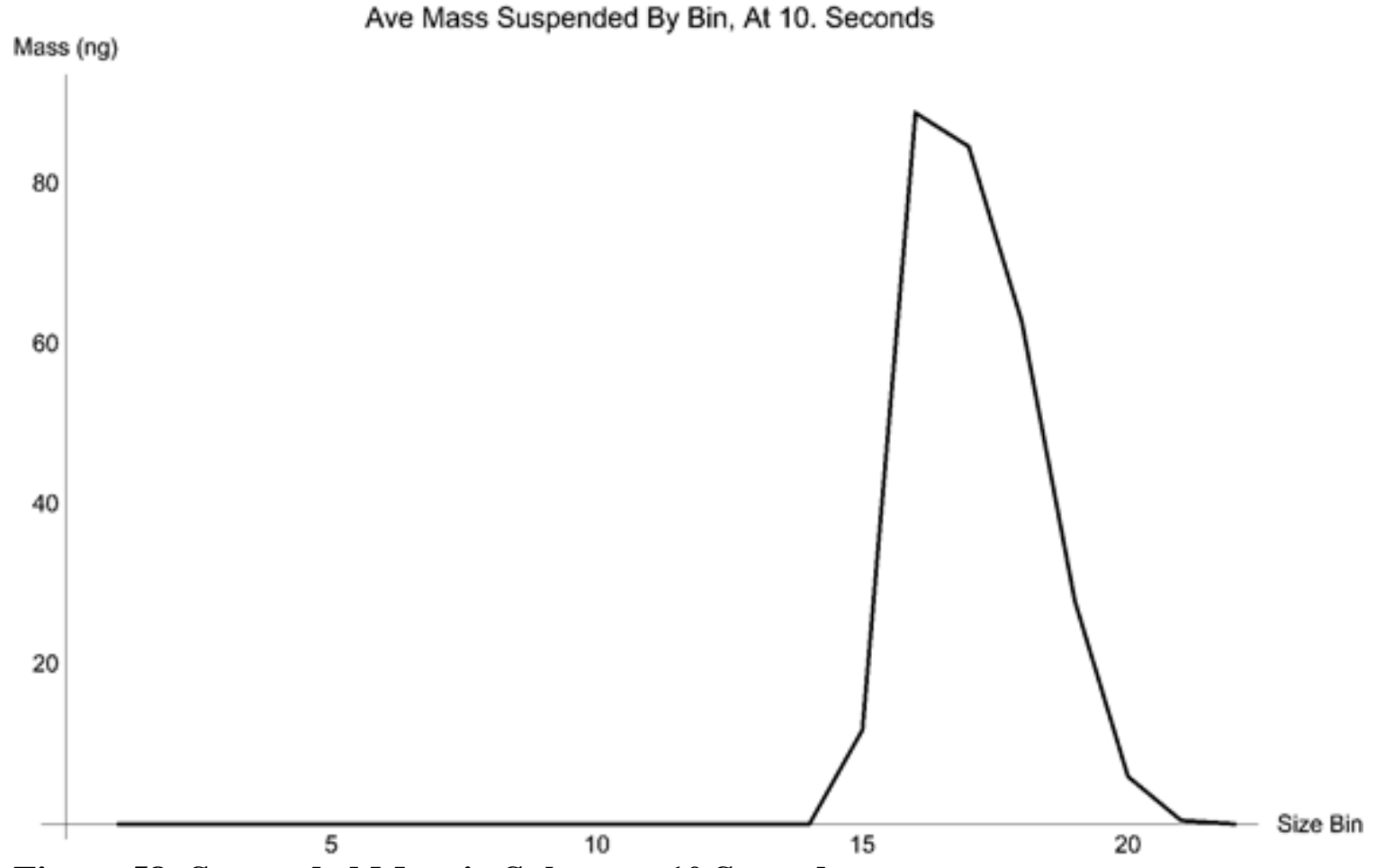

Figure 58: Suspended Mass in Sphere at 10 Seconds

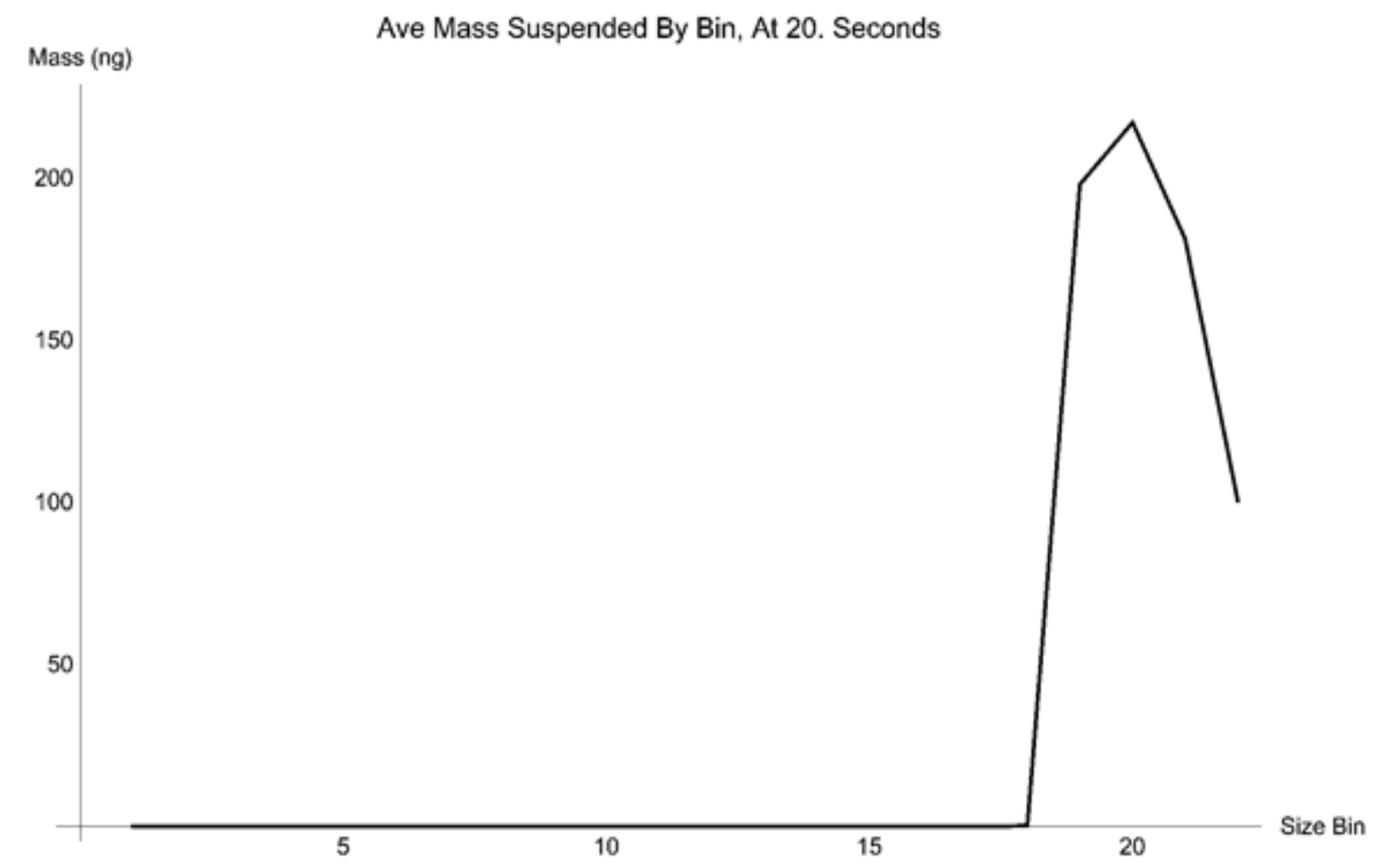

Figure 59: Suspended Mass in Sphere at 20 Seconds 


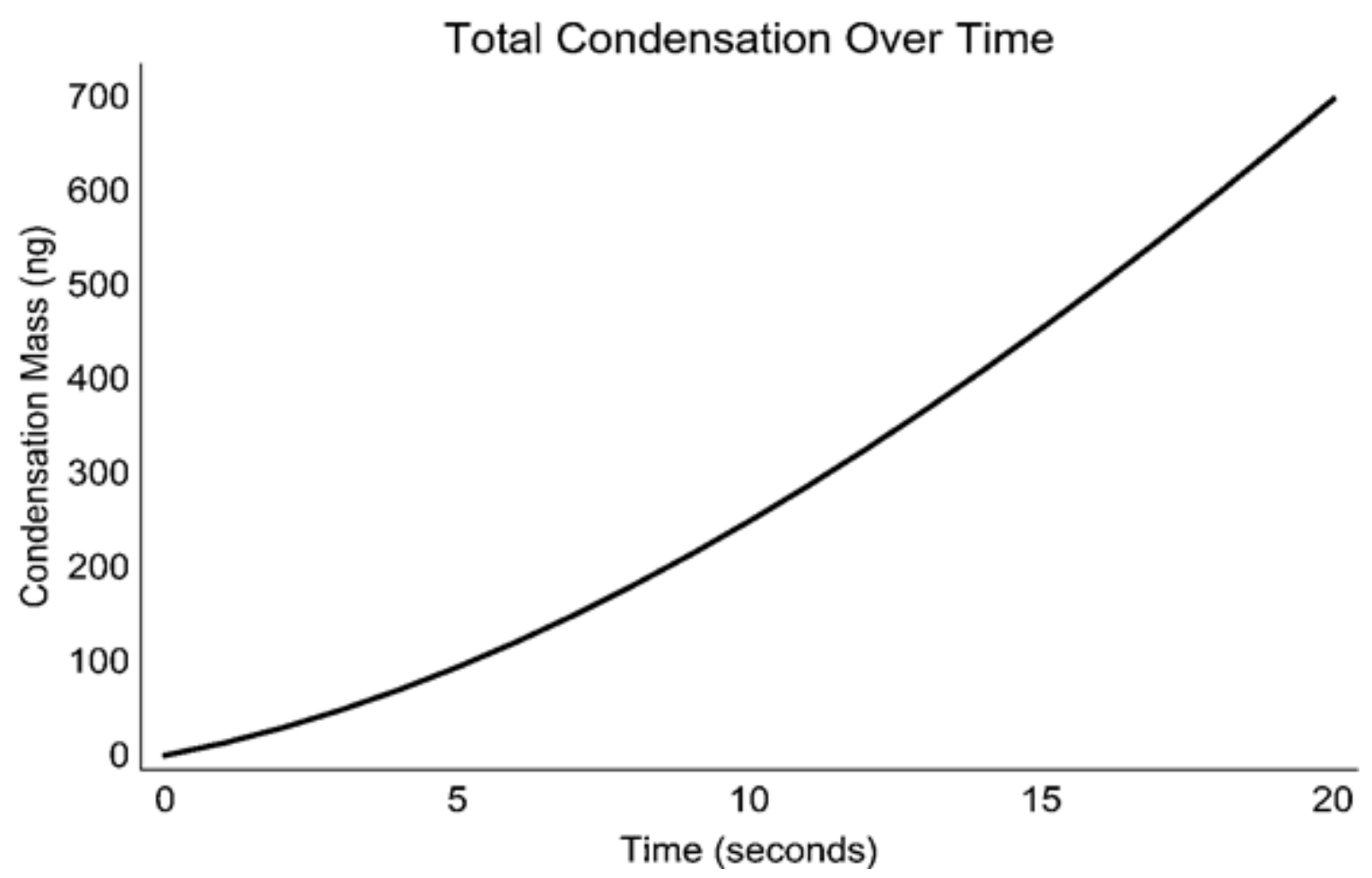

Figure 60: Total Condensation Inside of the Sphere

Unfortunately, due to the nature of the condensation process in the aerosol model, the cumulative particle deposition on the inner surface of the vessel could not be calculated. Instead, the total amount of condensation inside the vessel was tracked as a function of time. The results can be seen in Figure 60. In this figure, the total condensation initially climbs slowly, but bends upward as time progresses. This was due to the condensation process itself: Since the condensation rate is dependent on particle radius, the increasing particle size also increased the condensation rate.

\section{Cuboid}

After completing the modeling for the spherical vessel, the cuboid was integrated into the program. This was because of the increased complexity of the cuboid due to its physical discontinuities. The average mass concentration was calculated at $t=10$ seconds and $t=20$ seconds, with the results given in Figure 61 and Figure 62, respectively. Due 
to the vessel shape, the "Sample Sphere" technique was employed along the negative $y$ axis. In the first figure, the mass concentration is approximately $500 \mathrm{mg} / \mathrm{m}^{3}$. However, by the time the simulation has reached $t=20$ seconds, the average mass concentration has grown by about three times. Additionally, the range of concentration values has broadened. This is attributed to the condensation and coagulation effects of the DSMC method.

Once again, the significance of the condensation effect for the simulation can be further recognized by comparing the two mass concentration graphs to the data provided in Figure 32 and Figure 33 (the "Deposition Only" case for the same geometry). Just as with the spherical vessel, the mass concentration in these figures maintains a value of approximately $60 \mathrm{mg} / \mathrm{m}^{3}$. Since that case runs five times longer, the dramatic difference in mass concentration is attributed to the presence of condensation in the model.

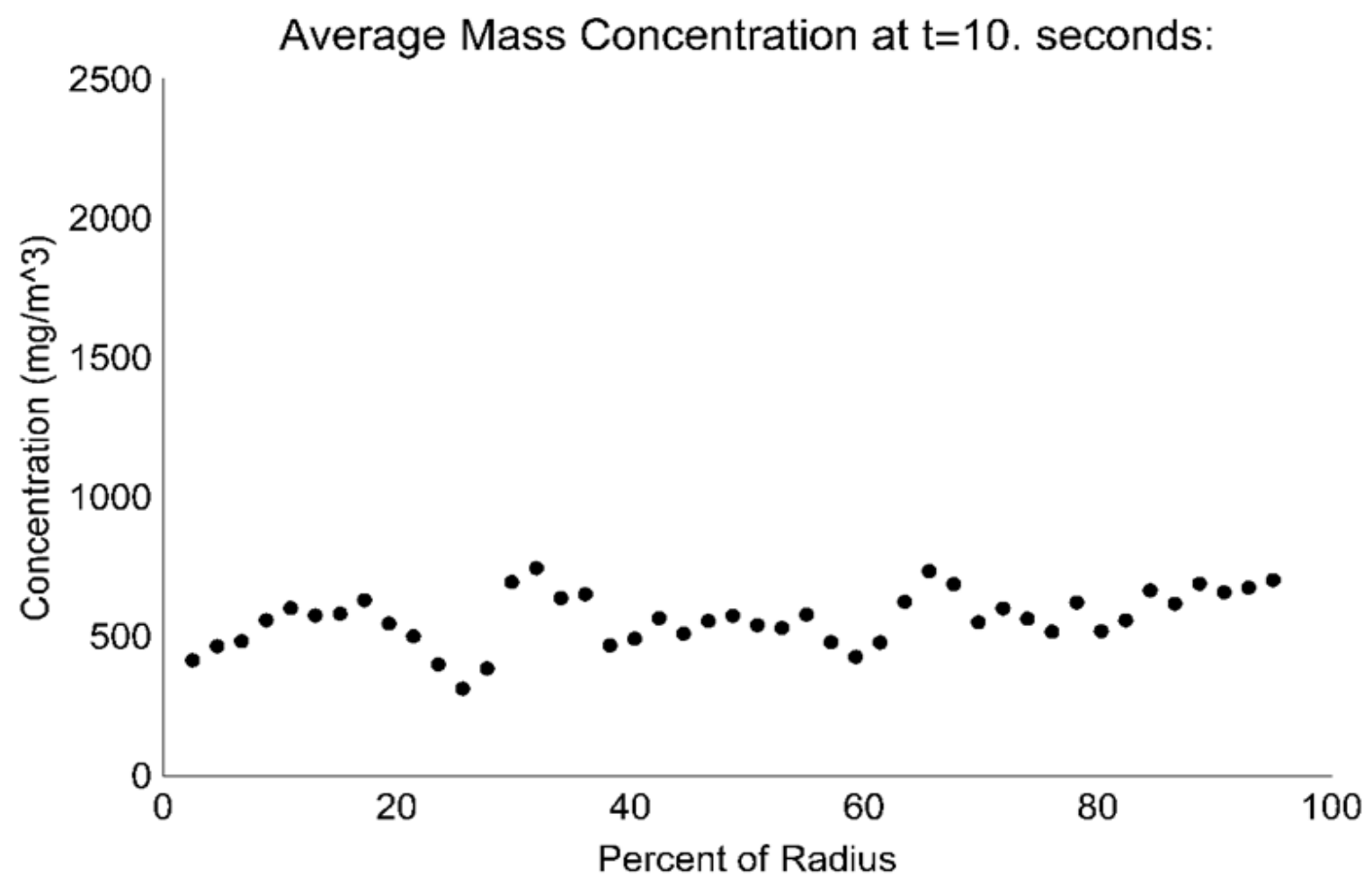

Figure 61: Mass Concentration of Cuboid at 10 Seconds 


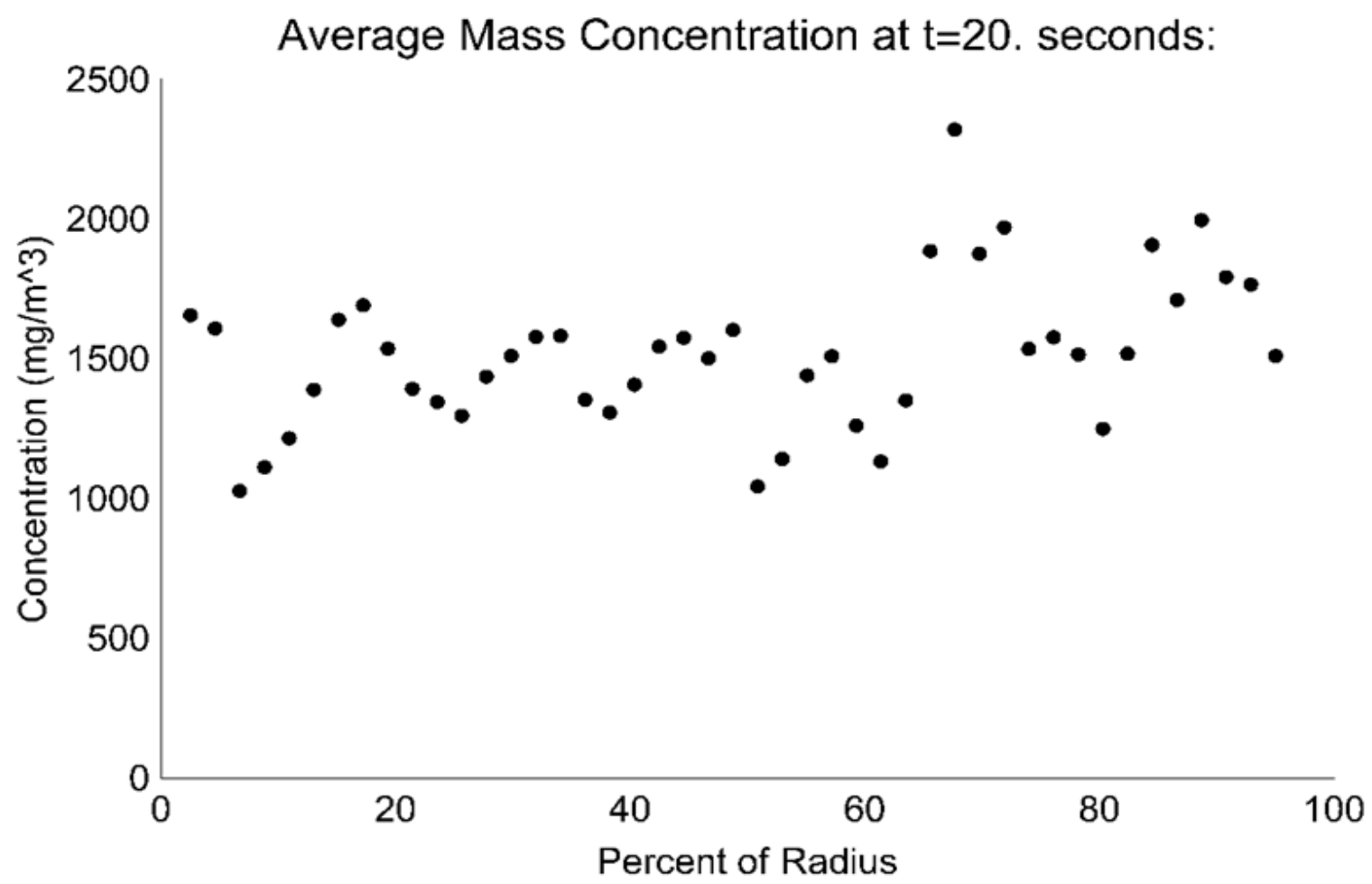

Figure 62: Mass Concentration of Cuboid at 20 Seconds

The average suspended mass for this vessel is given in Figure 63 and Figure 64 for $t=10$ seconds and $t=20$ seconds, respectively. For Figure 63, the amount of suspended mass peaks at approximately $170 \mathrm{ng}$ in the $16^{\text {th }}$ size bin. This corresponds to aerosol particles $0.246 \mu \mathrm{m}$ to $0.277 \mu \mathrm{m}$ in diameter. In the next graph, the suspended mass peak is in the $20^{\text {th }}$ size bin with a value a little over $400 \mathrm{ng}$. Just like with the spherical vessel, the total overall shift is four size bins, three more than in the "Deposition and Coagulation" case for the same time duration. Since all of the variable values from the deposition and coagulation processes were carried over from previous simulations with the cuboid, they could not have caused the increased peak shift. Note that, once again, a portion of the line in the graph is missing. To resolve this, the maximum size bin allowed in the program would need to be adjusted. This remains an area of potential future work. 


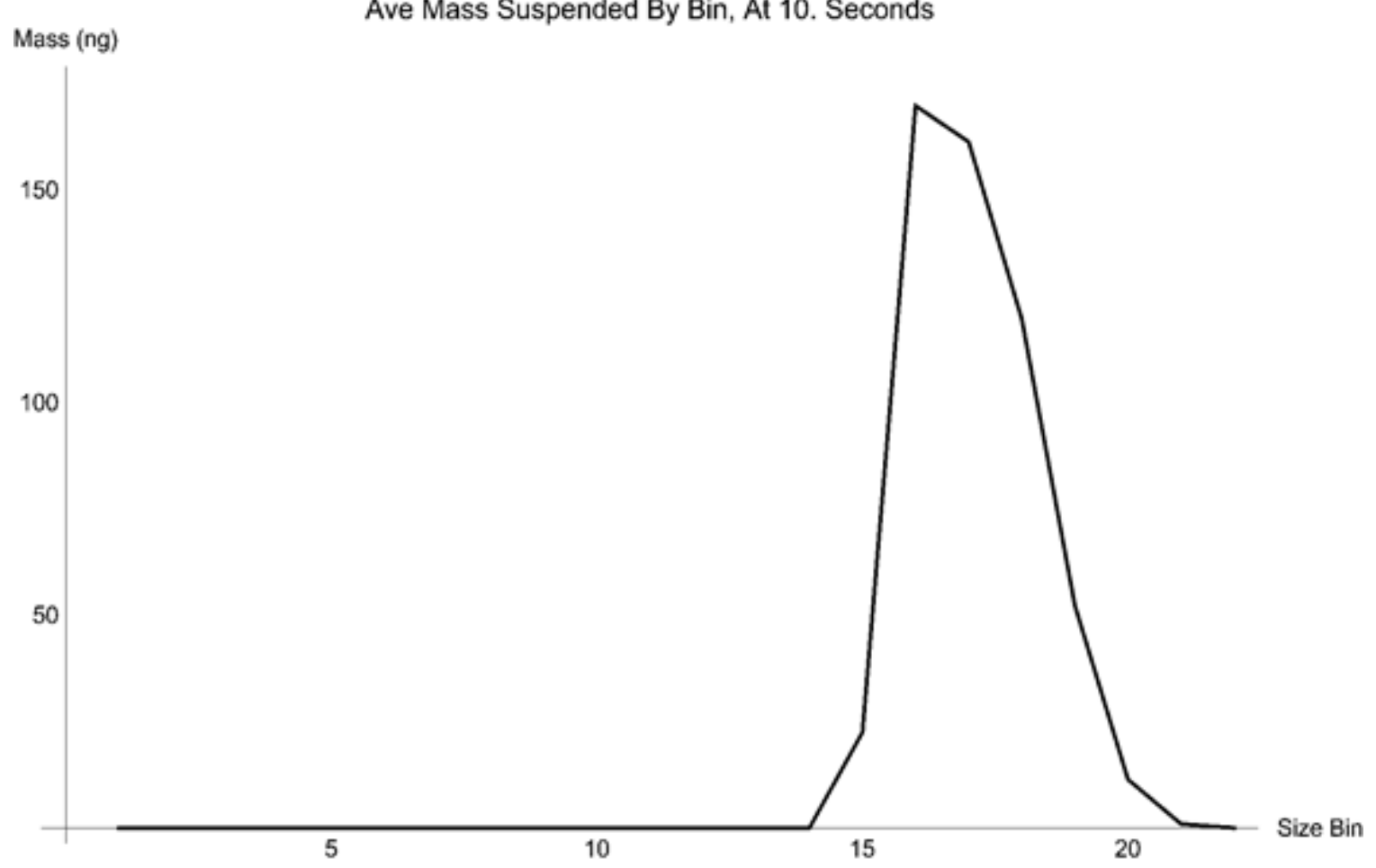

Figure 63: Suspended Mass in Cuboid at 10 Seconds

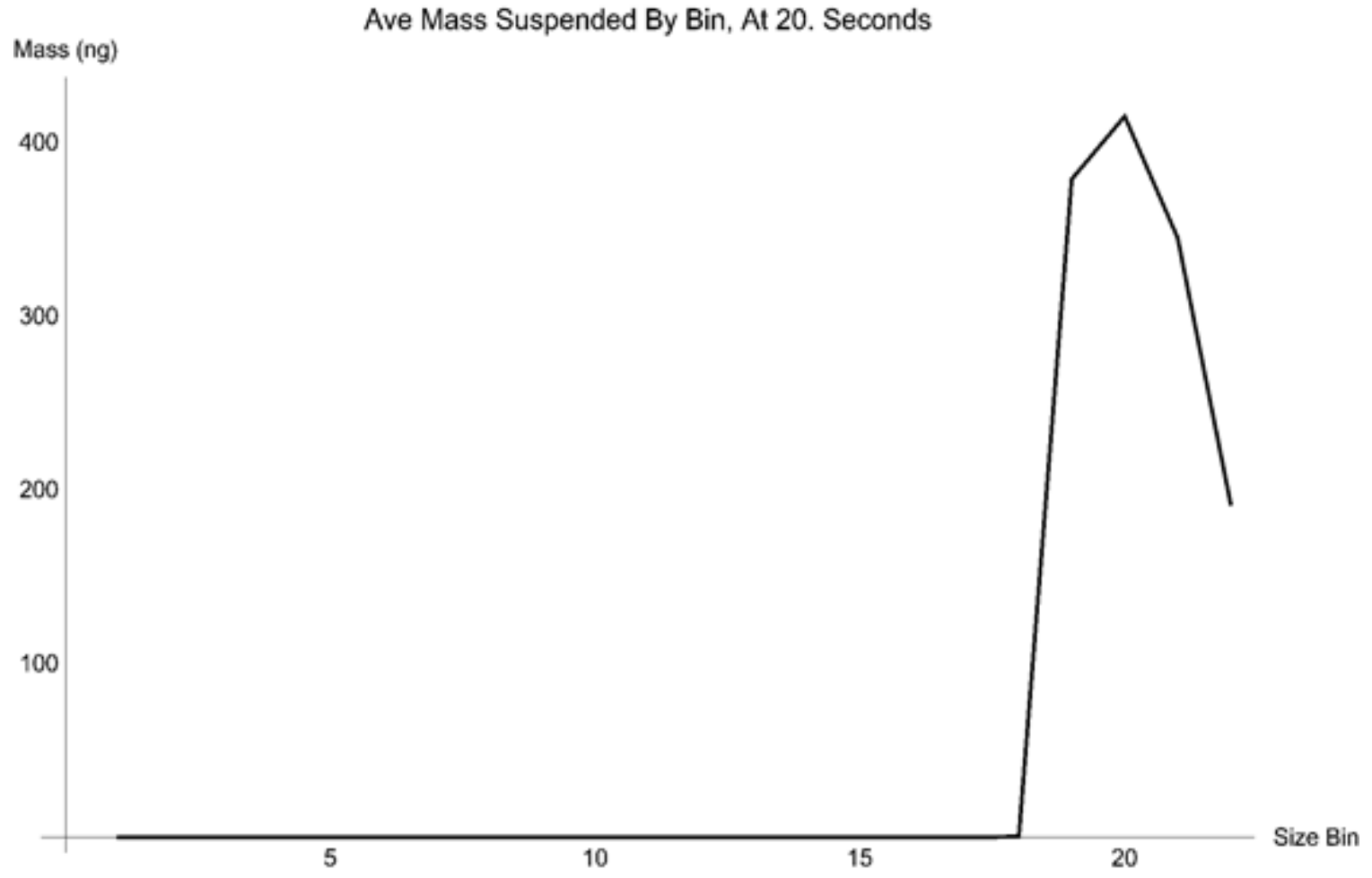

Figure 64: Suspended Mass in Cuboid at 20 Seconds 


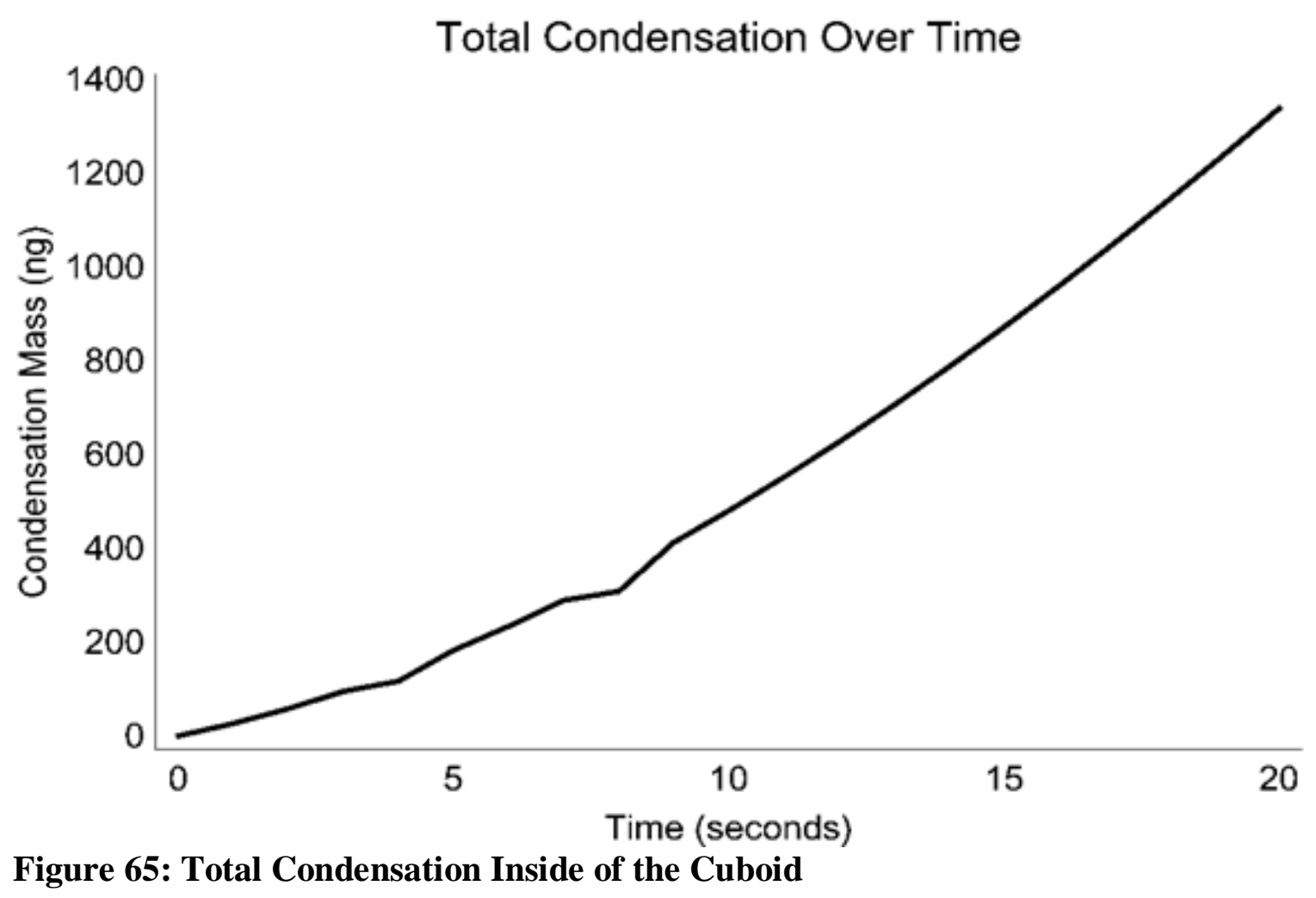

Just like with the previous geometry, the total amount of condensation inside the vessel was tracked as a function of time. Figure 65 shows these results. For this figure, the initial condensation rate is low. However, it increases as time progresses. The overall effect is the upward curve shown in the figure. This is due to the fundamental nature of the condensation process: The condensation rate is dependent on particle radius. As a result, increasing the particle size also increases the condensation rate. By the time the simulation ends at $t=20$ seconds, approximately $1400 \mathrm{ng}$ of water have condensed onto the aerosol particles in the simulation.

\section{Sphere with Internal Obstruction}

The last vessel tested for the deposition, coagulation, and condensation aerosol model was the sphere with an internal obstruction. Figure 66 and Figure 67 correspond to the average mass concentration at $t=10$ seconds and $t=20$ seconds, respectively. To 
determine average mass concentration, the "Sample Sphere" technique was used along the positive $x$ axis.

Recall that, in this direction, the spherical obstruction extends a distance of $1.5 R_{\text {Obs }}$ from the origin. Since $R_{O b s}=R / 4$, the distance can be rewritten as $R_{O b s}=3 R / 8$ (37.5\% of the total radial distance). As discussed previously, this means that no particles in Figure 66 or Figure 67 should appear before $37.5 \%$ of the total distance. However, both figures indicate that the local mass concentration starts increasing slightly before this point. The reason for this is due to the "Sample Sphere" calculation method. With the technique, it is possible for a sphere centered just outside a given vessel boundary to have a radius large enough to incorporate some aerosol particles inside the vessel into its calculations.

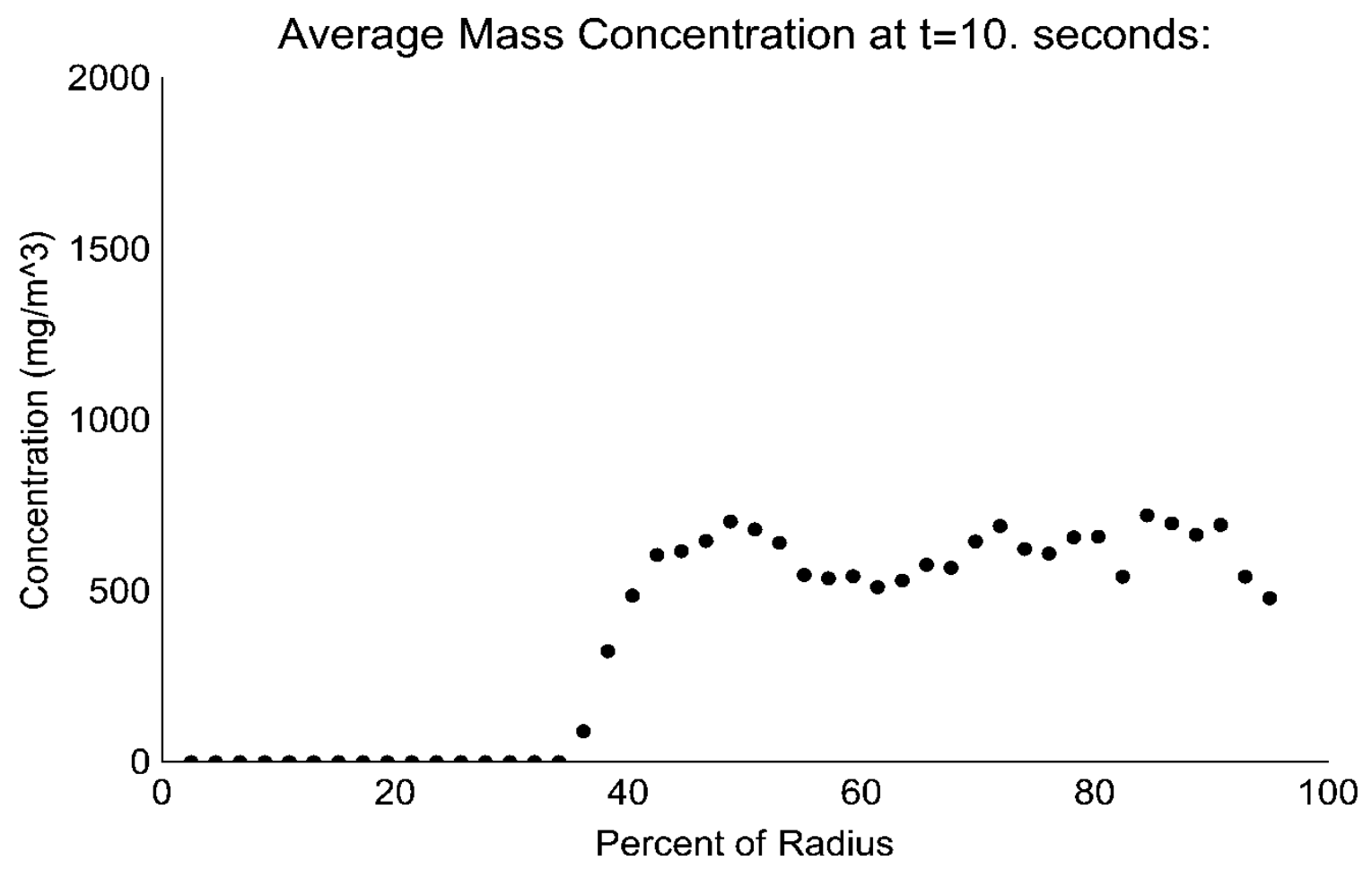

Figure 66: Mass Concentration of Sphere with Internal Obstruction at 10 Seconds 


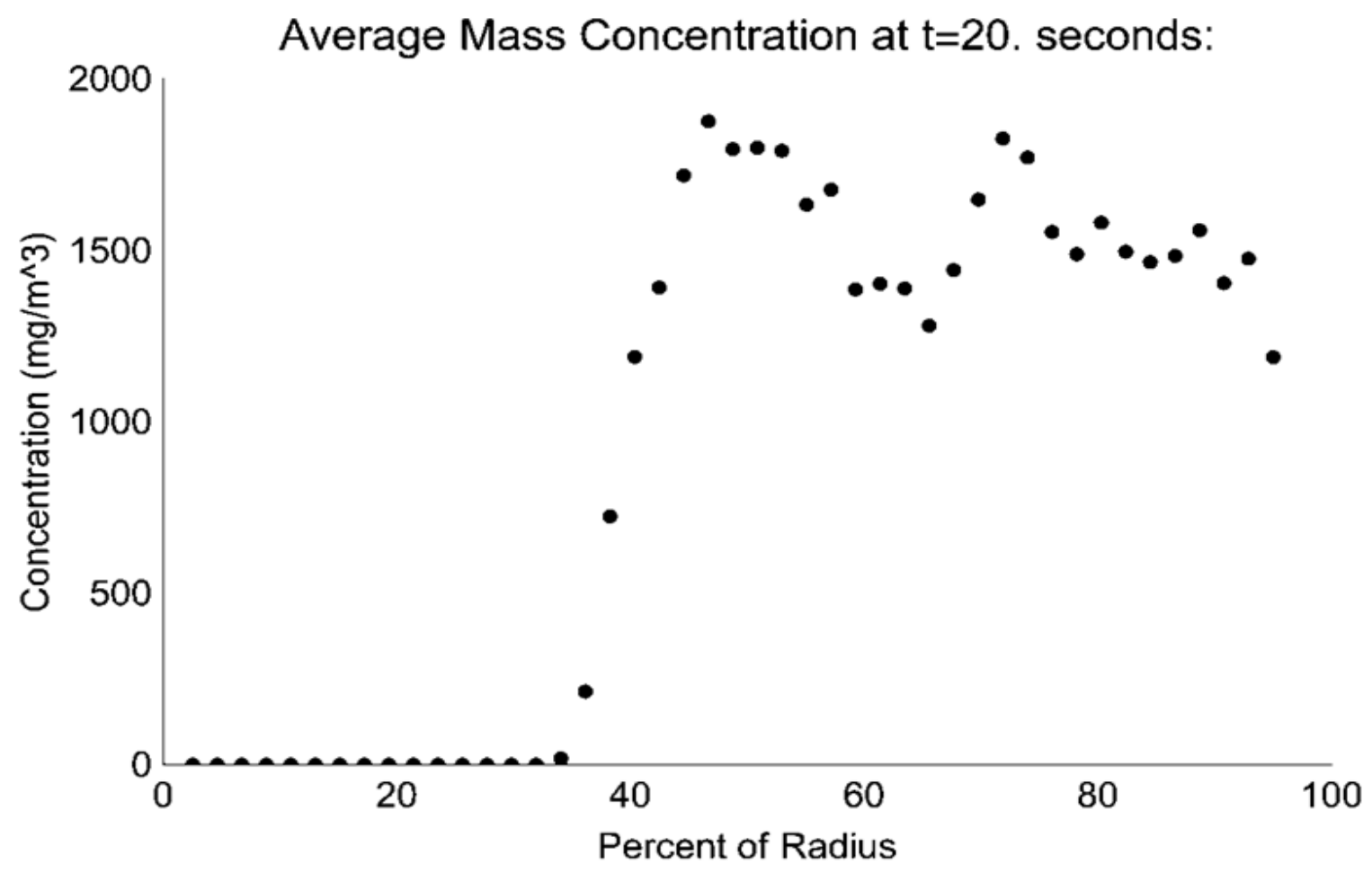

Figure 67: Mass Concentration of Sphere with Internal Obstruction at 20 Seconds

After completing computations for the mass concentration, the average suspended mass at $t=10$ seconds and $t=20$ seconds was calculated. The results of these calculations are shown in Figure 68 and Figure 69. In the first figure, the amount of suspended mass peaks at approximately $85 \mathrm{ng}$ in the $16^{\text {th }}$ size bin. This corresponds to aerosol particles $0.246 \mu \mathrm{m}$ to $0.277 \mu \mathrm{m}$ in diameter. The next figure (Figure 69) has a suspended mass peak at the $20^{\text {th }}$ size bin with a value of about $210 \mathrm{ng}$. This corresponds to aerosol particles $0.399 \mu \mathrm{m}$ to $0.450 \mu \mathrm{m}$ in diameter. None of the parameters affecting deposition or coagulation were changed from the "Deposition and Coagulation" aerosol case for this vessel. As a result, the increased peak shift can be solely attributed to condensation. Note that, just as with the previous geometries, a portion of the line in the graph is missing. To resolve this, the distribution of particles sizes (and their associated size bins) would need to be changed. This remains an area of potential future work. 


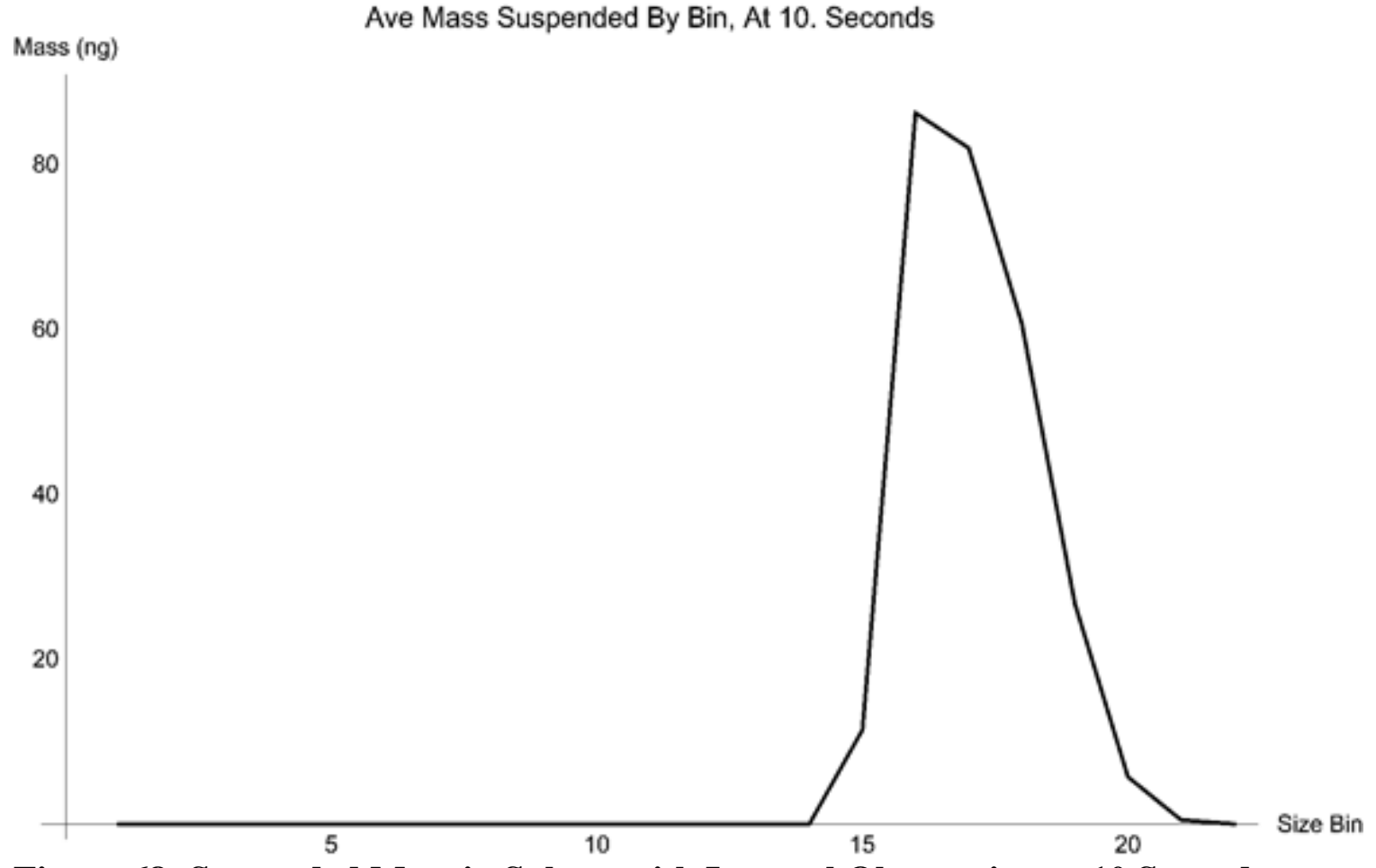

Figure 68: Suspended Mass in Sphere with Internal Obstruction at 10 Seconds

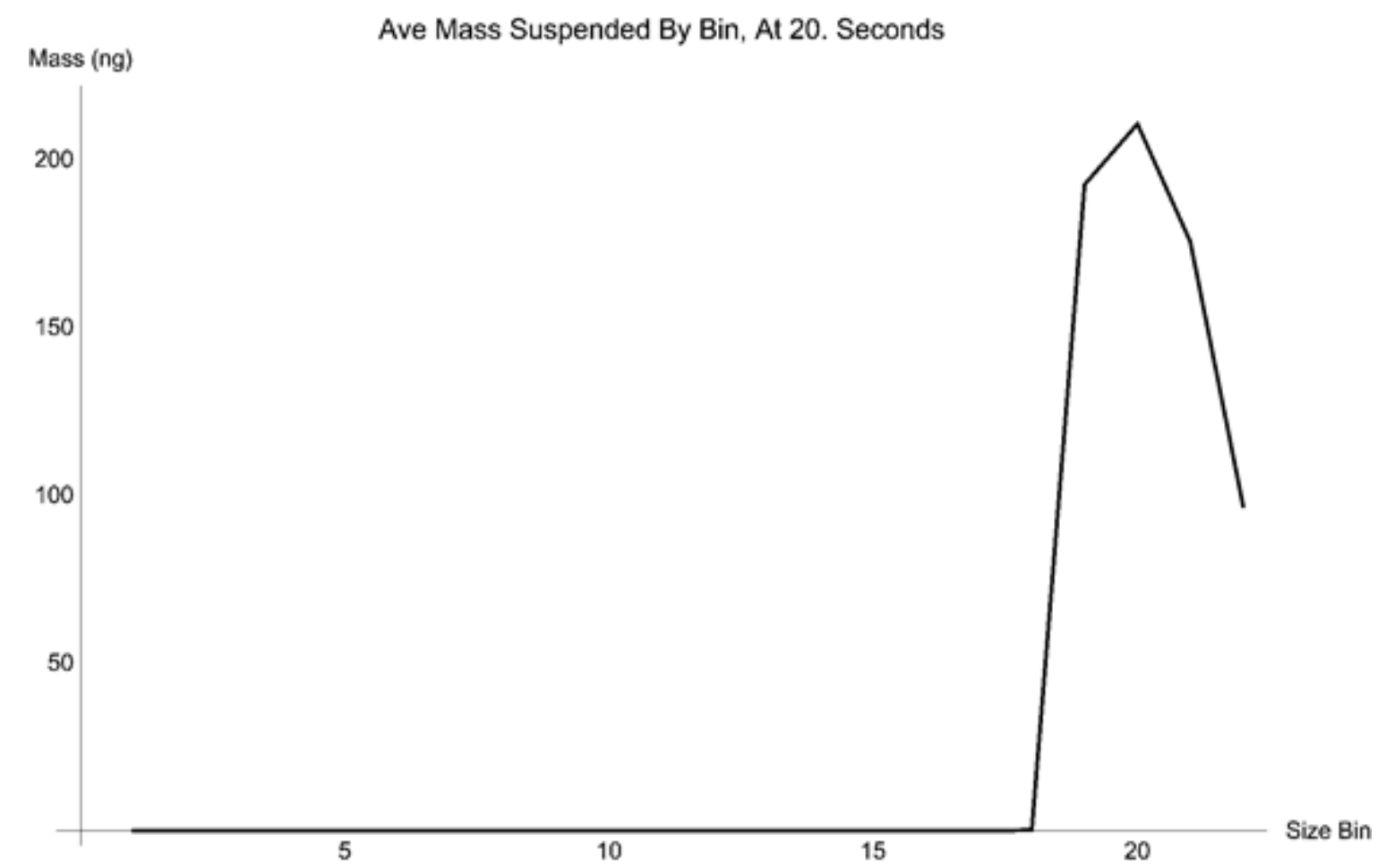

Figure 69: Suspended Mass in Sphere with Internal Obstruction at 20 Seconds 


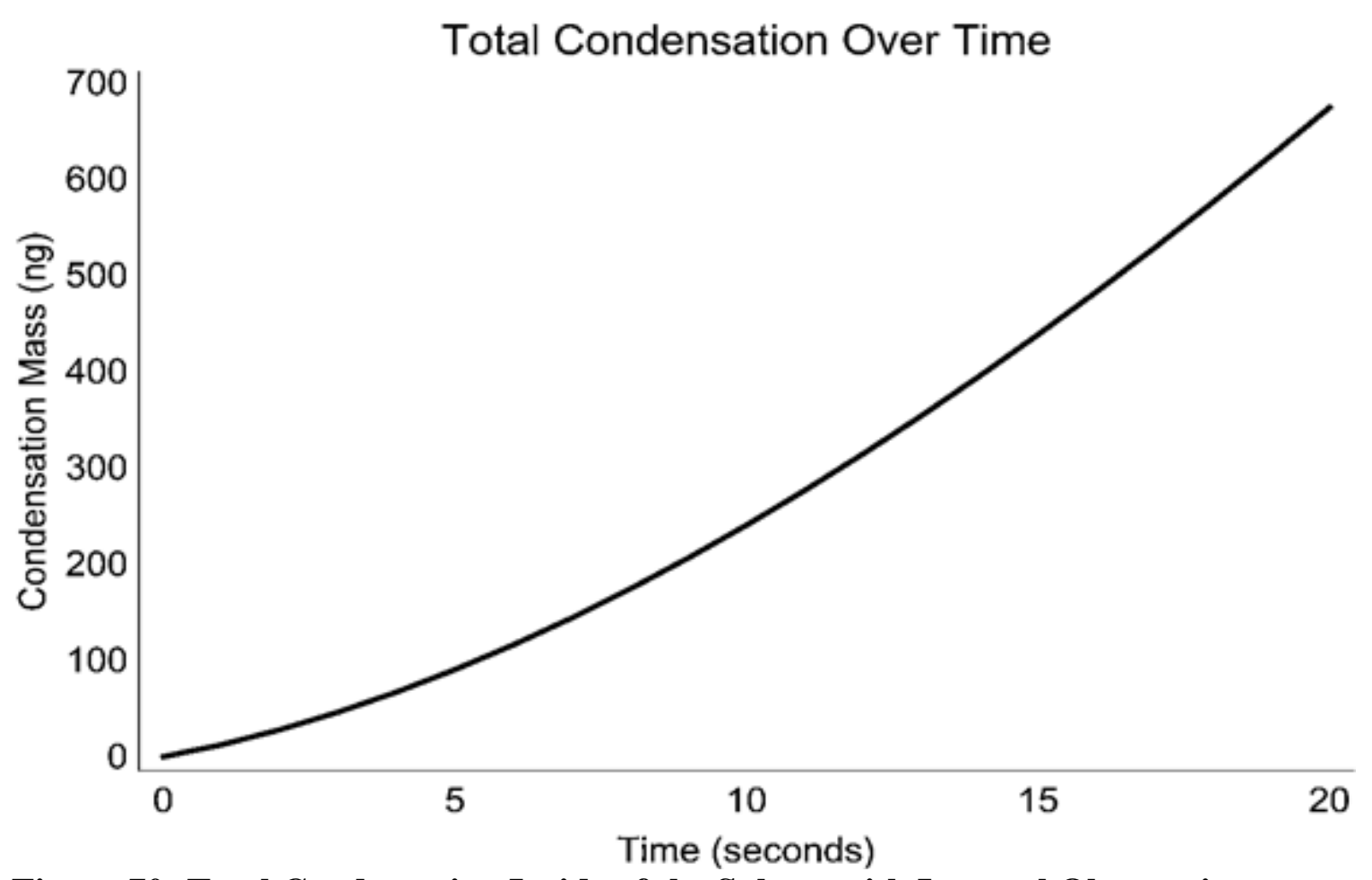

Figure 70: Total Condensation Inside of the Sphere with Internal Obstruction

In addition to the suspended mass distribution, cumulative condensation was also determined. Total condensation over time for the vessel is shown in Figure 70. In this graph, the condensation behavior is identical to that in the previous two vessels. Initially, the condensation grows slowly. As the total elapsed time increases, the growth rate increases. The overall effect is the upward curve shown in the figure. It is critical to recall that the reason for this curve is because the condensation rate is dependent on particle radius. This means that as the particle size increases, the condensation rate will also. By the time the simulation ends at $t=20$ seconds, approximately $675 \mathrm{ng}$ of water have condensed onto the aerosol particles in the simulation. 


\section{SUMMARY}

For this work, a spatially inhomogeneous aerosol model was developed in Wolfram Mathematica. It utilized a mesh-free recursive particle clustering method known as PAM to replace the more traditional mesh-based DSMC technique. The PAM approach takes representative points (medoids) and then clusters the particles based on their distance to these points. Particles within each cluster are then allowed to interact with each other. This technique makes the program less dependent on vessel geometry, and will show its true value in situations where the vessel complexity limits the quality of the traditional mesh-based DSMC method.

To verify the accuracy of the aerosol model, a simulation was created to replicate the deposition and coagulation behaviors determined in previous literature. In order to reproduce the results generated by Campbell et al. ${ }^{33}$, a spherical geometry was used as the first vessel in the program. This shape generated data for both the "Deposition Only" case as well as the "Deposition and Coagulation" case. Both results were compared with the information provided in the work by Campbell et al., and it was determined that the aerosol model generated similar values.

After verifying the model against prior work, additional geometries were tested for deposition behavior. These shapes included an ellipsoid, torus, elliptical cylinder, cuboid, and sphere with an internal obstruction. For each of these vessels, the mass concentration maintained a value of approximately $60 \mathrm{mg} / \mathrm{m}^{3}$. However, as the interior boundary was approached, the concentration declined for each shape. This was due to the deposition effects caused by the interaction of the aerosol particles with the interior boundary of the geometry. Additionally, the cumulative particle deposition was graphed. 
For the sphere and ellipsoid, the results were compared with FEM calculations to verify the program's accuracy. The close correspondence between the DSMC and FEM values demonstrated the accuracy of the spatially inhomogeneous aerosol model. In each figure, the cumulative deposition climbs quickly for the first five seconds (5\% of the total simulation time) and then the deposition rate slows and the cumulative deposition tapers off. All the other geometries exhibited similar behavior.

Once the deposition simulations were done for each of the additional geometries, coagulation was added to the spatially inhomogeneous aerosol model. This combination of aerosol processes was applied to an ellipsoid, torus, elliptical cylinder, cuboid, and sphere with an internal obstruction. For each geometry, the suspended particle size distribution was evaluated at $t=10$ seconds and $t=20$ seconds. The amount of suspended mass for the first evaluation time peaked in the $11^{\text {th }}$ size bin, which corresponded to aerosol particles of $0.134 \mu \mathrm{m}$ to $0.151 \mu \mathrm{m}$ in diameter. Suspended mass for the second evaluation time peaked in the $12^{\text {th }}$ size bin, which corresponded to aerosol particles of $0.151 \mu \mathrm{m}$ to $0.171 \mu \mathrm{m}$ in diameter. Since there was no condensation effects present in this simulation, the peak shift was solely dependent on the coagulation process in the aerosol model.

Finally, when all the previous modeling had been completed, condensation was incorporated into the program. Unlike the previous two cases, modeling all three aerosol processes was limited to the inside of a sphere, cuboid, and sphere with internal obstruction. Due to the complexity, the program duration was kept at a total of 20 seconds, with the output data evaluated at $t=10$ seconds and $t=20 \mathrm{~seconds}$. For each vessel, the local mass concentration at $t=10$ seconds was approximately $500 \mathrm{mg} / \mathrm{m}^{3}$. The 
size range of concentration values at $t=20$ seconds broadened considerably, but appeared to be centered around $1500 \mathrm{mg} / \mathrm{m}^{3}$. This increased size range for these particles was attributed to the condensation and coagulation effects of the DSMC method.

In addition to the local mass concentration graphs, the size distribution for suspended aerosol particles was plotted at both evaluation times. The two resulting figures demonstrated that the size distribution peak shifted significantly to the right as time progressed. For each of the three geometries tested, this shift was four particle size bins in magnitude. The rapid increase in particle size outpaced the effects of coagulation, demonstrating the significance of condensation in the program.

The significance of the condensation phenomenon was further verified by plotting the total amount of condensation over time for each of the three geometries. In these figures, the total condensation curved upward as time progressed, indicating an increasing condensation rate. This was due to the condensation process itself: Since the condensation rate is dependent on particle radius, the increasing particle size also increased the condensation rate. The shape of these graphs followed expectations. 


\section{CONCLUSIONS}

In the work described above, a mesh-free DSMC aerosol model was constructed. This program was generalized to accommodate arbitrary vessel geometries and then tested so as to compare results with previously published literature and other approximation techniques. After comparison with other calculations, it is definitive that the mesh-free DSMC aerosol program constructed in the preceding sections successfully expands existing aerosol modeling capabilities.

The first expansion in modeling capability due to the DSMC code is in the ability to model deposition, coagulation, and condensation processes inside arbitrary vessel boundaries. Incorporation of all three processes for a generalized multicomponent aerosol had previously never been accomplished using the DSMC technique. Additionally, the vessel boundaries were successfully generalized so as to be able to model an aerosol inside any vessel geometry defined by a surface equation or series of surface equations. This eliminated symmetry restrictions that had previously limited research in this area and allowed for the construction of complex geometries, including reactor vessels.

A second major expansion in modeling capability was in regards to limitations imposed on the user. Unlike MAEROS, the current modeling standard, the mesh-free DSMC aerosol model did not limit particle complexity or enforce phenomena restrictions. It also did not force non-physical assumptions limiting the model's accuracy. Instead, the DSMC program provided the user with an unprecedented amount of control over what considerations were made within the model.

The last tremendous advantage that the DSMC aerosol modeling program provides is geometry independence. Unlike most other methods, the mesh-free technique 
employed in this work depended solely on the Euclidian distance from each particle to the nearest medoid point. This separated the required computational time from the vessel shape. Alternative approaches require the computation of aerosol particles inside mesh cells, which are dependent on the shape of the geometry. The dependence can quickly become computationally demanding as well as extremely labor intensive, depending on the enclosing geometric boundaries of the computational domain.

Due to the successful generalization of the DSMC aerosol program, there are many potential areas of future work. One such area should concentrate on modeling the remaining three fundamental geometries (the ellipsoid, torus, and elliptical cylinder) for the deposition, coagulation, and condensation case. A second area worth future improvement would be modeling additional complex shapes. This would be done with the goal of being able to successfully model a complete reactor vessel geometry. Also, attention should be given to the existing deposition model. Currently, the model assumes that once a particle encounters the boundary, it deposits. However, a more accurate model would consider the effects of deposition probability and particle resuspension. Lastly, additional focus could be given to boundary modeling. For the current program, the vessel boundaries are considered to be "absolute" in that aerosol particles cannot pass beyond them. However, the program modeling could be extended to situations involving a "soft" boundary, such as a cloud or plume. 


\section{REFERENCES}

1. Williams M.M.R., Loyalka S.K., (1991), Aerosol Science: Theory and Practice with Special Applications to the Nuclear Industry, 1st ed. Pergamon Press, Oxford; New York.

2. Murata K.K., Williams D.C., Griffith R.O., Gido R.G., Tadios E.L., Davis F.J., Martinez G.M., Washington K.E., Tills J., (1997), Code Manual for CONTAIN 2.0: A Computer Code for Nuclear Reactor Containment Analysis. No. SAND97173. Sandia National Laboratories, Albuquerque, NM.

3. Humrickhouse P.W., (2011), HTGR Dust Safety Issues and Needs for Research and Development. Idaho National Laboratory (INL), Idaho National Laboratory.

4. Morris R.N., (2008), Next Generation Nuclear Plant Phenomena Identification and Ranking Tables (PIRTs) Volume 3: Fission-Product Transport and Dose PIRTs. Oak Ridge National Laboratory (ORNL), Oak Ridge National Laboratory.

5. Gauntt R.O., Cole R.K., Erickson C.M., Gido R.G., Gasser R.D., Rodriguez S.B., Young M.F., (2001), MELCOR Computer Code Manuals Vol. 3: Demonstration Problems. Sandia National Laboratories.

6. Moe W., (2010), HTGR Mechanistic Source Terms White Paper. Idaho National Laboratory (INL), Idaho National Laboratory.

7. Friedlander S.K., (2000), Smoke, Dust, and Haze: Fundamentals of Aerosol Dynamics, Second ed. Oxford University Press, New York.

8. Fuchs N., (1964), The Mechanics of Aerosols. Pergamon, New York.

9. Benson C., Newland M., (1996), Interactions of Fission Product Vapours with Aerosols.

10. Palaniswaamy G., Loyalka S.K., (2007), Direct Simulation Monte Carlo Multicomponent Aerosol Dynamics: Coagulation, Deposition, and Source Reinforcement. Nuclear Technology 160, 187-204.

11. Allelein H.-J., Auvinen A., Ball J., Güntay S., Herranz L.E., Hidaka A., Jones A.V., Kissane M., Powers D., Weber G., (2009), State of the Art Report on Nuclear Aerosols, Organization for Economic Cooperation and Development: Committee on the Safety of Nuclear Installations. Nuclear Energy Agency.

12. Campbell S.A., Loyalka S.K., (2015), Computation of Aerosol Evolution Under Coagulation. Nuclear Science and Engineering 181, 137-159. 
13. Clement C., Harrison R., (2000), Enhanced Localised Charging of Radioactive Aerosols. Journal of Aerosol Science 31, 363-378.

14. Ramabhadran T.E., Peterson T.W., Seinfeld J.H., (1976), Dynamics of Aerosol Coagulation and Condensation. AIChE Journal 22, 840-851.

15. Wilson J.H., Arwood P.C., (1990), Comparison of (Postest) Predictions of Aerosol Codes with Measuraments in LWR Aerosol Containment Experiment (LACE) LA4.

16. Wright A., Arwood P., (1987), Summary of Posttest Aerosol Code Comparisons for LWR Aerosol Containment Experiment (LACE) LA3. Oak Ridge National Lab., TN (USA).

17. Wright A., Wilson J., Arwood P., (1986), Summary of Aerosol Code-Comparison Results for LWR (Light-Water Reactor) Aerosol Containment Tests LA1, LA2, and $L A 3$, Fourteenth Water Reactor Safety Meeting. Oak Ridge National Lab., TN (USA), Gaithersburg, Maryland.

18. Wright A., Wilson J., Arwood P., (1987), Summary of Posttest Aerosol Code Comparisons for LWR Aerosol Containment Experiment (LACE) LA1. Oak Ridge National Lab., TN (USA).

19. Wright A., Wilson J., Arwood P., (1989), Summary of Posttest Aerosol Code Comparisons for LWR Aerosol Containment Experiment (LACE) LA6. Oak Ridge National Lab., TN (USA).

20. Matijević E., Espenscheid W., Kerker M., (1963), Aerosols Consisting of Spherical Particles of Sodium Chloride. Journal of Colloid Science 18, 91-94.

21. Langer G., Pierrard J., (1963), Anomalous Behavior of Aerosol Produced by Atomization of Monodisperse Polystyrene Latex. Journal of Colloid Science 18, 95-97.

22. Wolfenbarger J.K., Seinfeld J.H., (1990), Inversion of Aerosol Size Distribution Data. Journal of Aerosol Science 21, 227-247.

23. Stempniewicz M.M., Winters L., Caspersson S.A., (2012), Analysis of Dust and Fission Products in a Pebble Bed NGNP. Nuclear Engineering and Design 251, 433-442.

24. Chabay I., Bright D.S., (1978), Measurement of the Size Distribution of Liquid and Solid Aerosols by Doppler Shift Spectroscopy. Journal of Colloid and Interface Science 63, 304-309.

25. Brock J.R., (1963), Forces on Aerosols in Gas Mixtures. Journal of Colloid Science 18, 489-501. 
26. Fierz M., Scherrer L., Burtscher H., (2002), Real-Time Measurement of Aerosol Size Distributions with an Electrical Diffusion Battery. Journal of Aerosol Science 33, 1049-1060.

27. Palsmeier J.F., Loyalka S.K., (2013), Evolution of Charged Aerosols: Role of Charge on Coagulation. Nuclear Technology 184, 78-95.

28. Gelbard F., (1983), MAEROS user manual. Sandia National Laboratory.

29. Gillespie T., (1963), The Effect of Size Distribution on the Rate Constants for Collisions in Disperse Systems. Journal of Colloid Science 18, 562-567.

30. Peterson T.W., Gelbard F., Seinfeld J.H., (1978), Dynamics of Source-Reinforced, Coagulating, and Condensing Aerosols. Journal of Colloid and Interface Science 63, 426-445.

31. Gelbard F., Seinfeld J., (1978), Coagulation and Growth of a Multicomponent Aerosol. Journal of Colloid and interface Science 63, 472-479.

32. Baron P.A., Willeke K., (2001), Aerosol Measurement: Principles, Techniques, and Applications. John Wiley \& Sons.

33. Campbell S.A., Palsmeier J., Loyalka S.K., (2016), Mesh-Free Simulation of Aerosol Evolution. Nuclear Science and Engineering 182, 287-296.

34. Park J., Loyalka S., (1989), Role of Spatial Inhomogeneities in Source Term Aerosol Dynamics. Nuclear Science and Engineering 101, 269-279.

35. Woods L.C., (1993), An introduction to the kinetic theory of gases and magnetoplasmas. Oxford University Press.

36. Bird G.A., (1976), Molecular Gas Dynamics. Clarendon Press, Oxford.

37. Bird G.A., (1994), Molecular Gas Dynamics and the Direct Simulation of Gas Flows, 2nd ed. Clarendon Press, Oxford.

38. Rousseeuw P.J., Kaufman L., (1990), Finding Groups in Data. Wiley Online Library.

39. Albalate A., Minker W., (2013), Semi-Supervised and Unervised Machine Learning: Novel Strategies. John Wiley \& Sons.

40. Farris R., (2016), U.S. Watches As Fukushima Continues To Leak Radiation, Accessed on January 31 2018, https://www.cnbc.com/2016/03/10/us-watches-asfukushima-continues-to-leak-radiation.html

41. Ono D., (2013), Fukushima Fallout: Should The West Coast Be Concerned?, Accessed on January 31 2018, http://abc7.com/archive/9317789/ 
42. Bowerman M., Loew T., (2016), Should We Be Worried About Fukushima Radiation? , Accessed on January 31 2018, https://www.usatoday.com/story/news/nation-now/2016/12/09/should-weworried-fukushima-radiation/95196156/

43. Saldivar I., De La Torre Aguilar F., Boraas M., Loyalka S.K., (2018), Benchmark problems in aerosol evolution: Comparison of some exact and DSMC results. Annals of Nuclear Energy 117, 213-222.

44. OECD-NEA, (1991), NESC1006 MAEROS - Multicomponent Aerosol Time Evolution, Accessed on February 2 2018, http://www.oecdnea.org/tools/abstract/detail/nesc1006/

45. Rangaraj D., Loyalka S.K., (2004), Direct Simulation Monte Carlo Aerosol Dynamics II - Role of Component Density Difference in Brownian Agglomeration, 2004 Annual Meeting - American Nuclear Society, June 13-17 2004. American Nuclear Society, Pittsburgh, PA, United States, pp. 301-302.

46. Palaniswaamy G., Loyalka S.K., (2008), Direct simulation, Monte Carlo, aerosol dynamics: Coagulation and condensation. Annals of Nuclear Energy 35, 485-494.

47. Palaniswaamy G., Loyalka S.K., (2007), Direct simulation Monte Carlo aerosol dynamics: Collisional sampling algorithms. Annals of Nuclear Energy 34, 13-21.

48. Hinds W.C., (1982), Aerosol Technology. John Wiley and Sons, New York.

49. Palaniswaamy G., Loyalka S.K., (2007), Direct Simulation Monte Carlo Aerosol Dynamics: Collisional Sampling Algorithms. Annals of Nuclear Energy 34, $13-$ 21.

50. Loyalka S., Park J., (1988), Aerosol Growth by Condensation: A Generalization of Mason's Formula. Journal of Colloid and Interface Science 125.

51. Holsoft, (2001), Online Physics Calculators, Accessed on February 92018 , https://www.holsoft.nl/physics/ocmain.htm

52. Mardia K., Kent J., Bibby J., (1979), Multivariate Analysis. Academic Press, London.

53. Loyalka S., Park J., (1988), Aerosol Growth by Condensation: A Generalization of Mason's Formula. Journal of Colloid and Interface Science 125, 712-716. 


\section{APPENDIX}

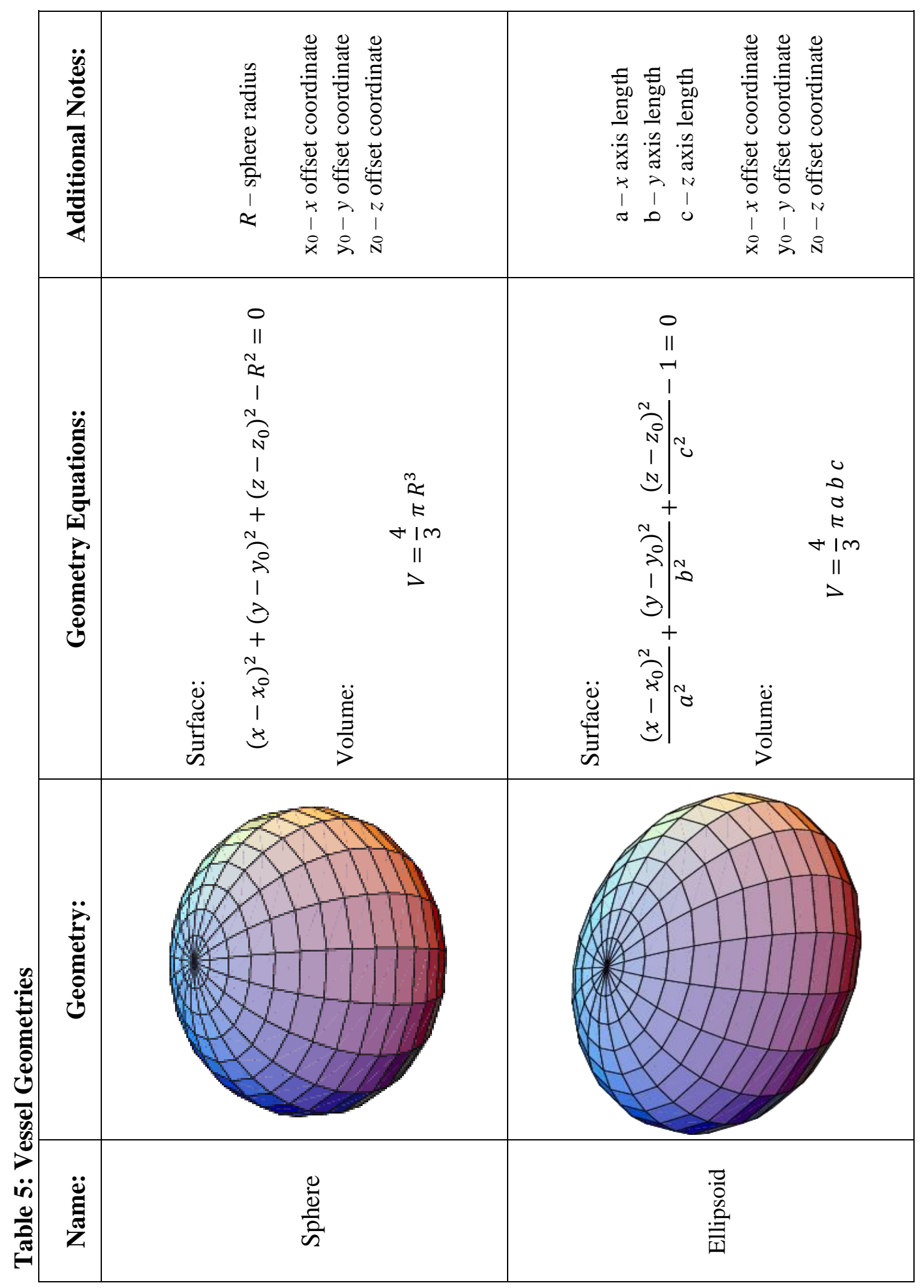




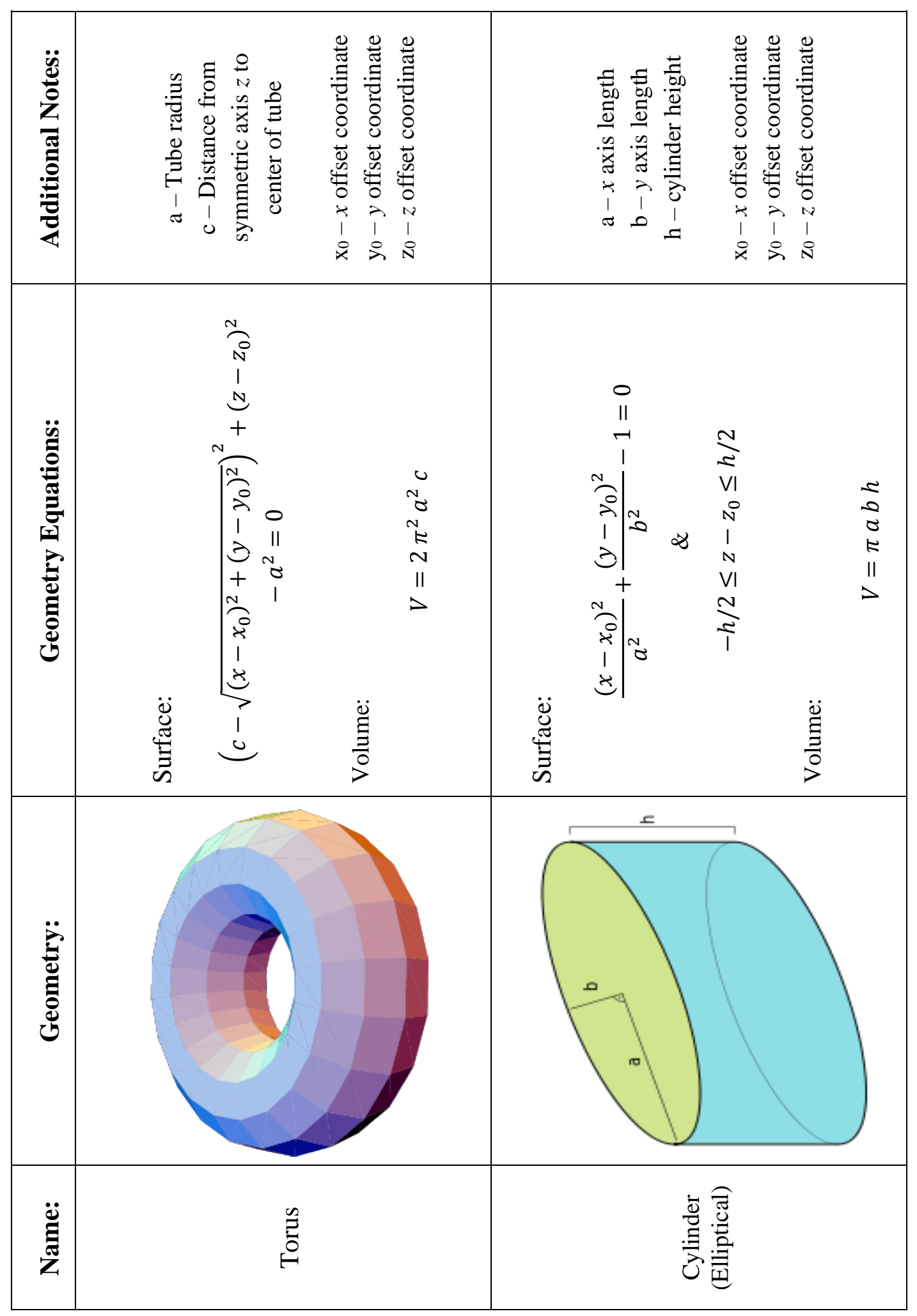




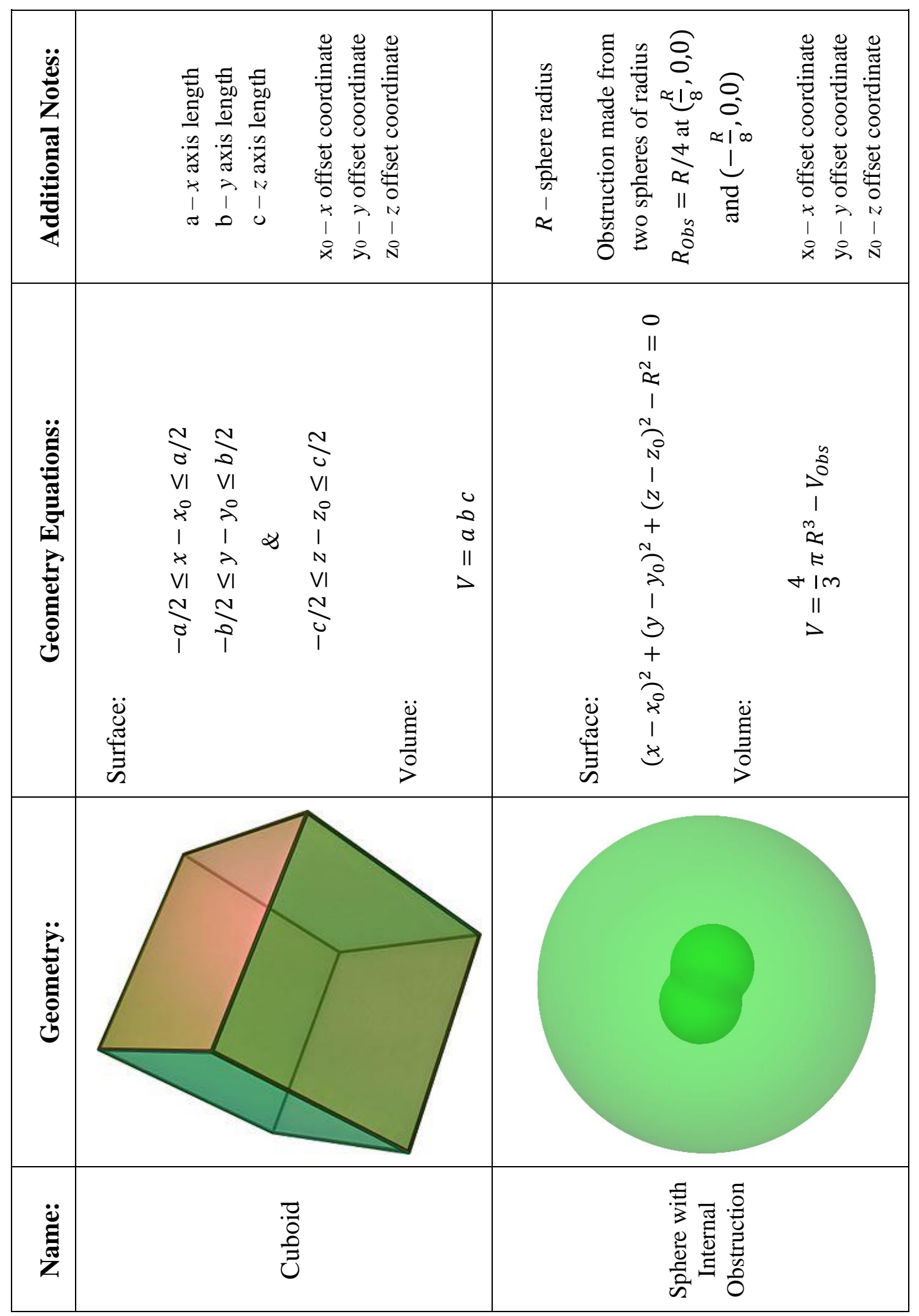




\section{VITA}

Matthew Boraas is a Nuclear Engineering PhD Candidate in the Nuclear Science Engineering Institute at the University of Missouri. His interests are in developing computational models for aerosol particles and their applications in the nuclear fuel cycle. More specifically, his graduate work focuses on modeling aerosols via the Direct Simulation Monte Carlo technique, using Wolfram Mathematica software.

He has recently coauthored the book Nuclear Batteries and Radioisotopes, as well as the article Thermal to Optical Energy Conversion: A Multi Megawatt Carbon Dioxide Laser Driven by an Extremely High Temperature Gas Cooled Reactor published in Progress in Nuclear Energy with Dr. Mark Prelas. Additionally, Matthew has coauthored the article Benchmark Problems in Aerosol Evolution: Comparison of Some Exact and DSMC Results published in the journal Annals of Nuclear Energy. He has also been the repeat recipient of the American Nuclear Society’s Saul Levine Memorial Scholarship in 2016 and 2017. 
